\title{
The Magnetic Properties of Selected Rare Earth Nitrides Grown by Pulsed Laser Deposition
}

\author{
by \\ B. M. Ludbrook
}

A thesis

submitted to the Victoria University of Wellington in fulfilment of the requirements for the degree of

Master of Science in Physics.

Victoria University of Wellington 2009 



\begin{abstract}
Gadolinium nitride $(\mathrm{GdN})$ and samarium nitride $(\mathrm{SmN})$ are grown by pulsed laser deposition on yttria stabilised zirconia substrates. Surface and structural characterisation shows that the thin films are epitaxial with crystallites of up to $30 \mathrm{~nm}$ in diameter and a very large in plane coherence length. A novel oxide layer is observed at the substrate-film interface, caused by oxygen in the substrate reacting with the deposited rare earth element. GdN is found to be ferromagnetic below $70 \mathrm{~K}$ with a saturation moment of $7 \mu_{B}$ per ion. The relationship between the crystal structure and the magnetisation is investigated using ferromagnetic resonance and a weak easy axis along the [111] azimuth is reported. Hall effect measurements show the carriers are electrons, present in concentrations of $10^{20} / \mathrm{cm}^{3}$. Magnetic measurements on $\mathrm{SmN}$ show the presence of metallic droplets, but correcting for these, the Curie temperature is found to be $30 \mathrm{~K}$. We report on preliminary growths of europium nitride and show the valence of the $\mathrm{Eu}$ is $3+$, solving an outstanding theoretical question.
\end{abstract}





\section{Contents}

1 Introduction 3

2 Experimental techniques $\quad 9$

2.1 Ferromagnetic resonance . . . . . . . . . . . . . . . 9 9

2.2 Magnetometry ... . . . . . . . . . . . . 13

2.3 Transport, Hall and magnetoresistance measurements . . . . 15

2.4 X-ray diffraction . . . . . . . . . . . . . . . . 17

2.5 Reflection high energy electron diffraction . . . . . . . . . . 18

3 Introduction to the magnetism of the rare-earth nitrides 21

4 Sample preparation $\quad 27$

4.1 Pulsed laser deposition . . . . . . . . . . . . . . . 27

4.2 Structural and surface characterisation . . . . . . . . . . . 34

4.3 Conclusions . . . . . . . . . . . . . . . . . 46

5 GdN $\quad 49$

5.1 Magnetometry in GdN . . . . . . . . . . . . . . . . . . . 49

5.2 Ferromagnetic resonance in $\mathrm{GdN} \ldots \ldots$. . . . . . . . 57

5.3 Magneto-transport in GdN . . . . . . . . . . . . . . . . . 65

$\begin{array}{lll}6 & \mathrm{SmN} & 73\end{array}$

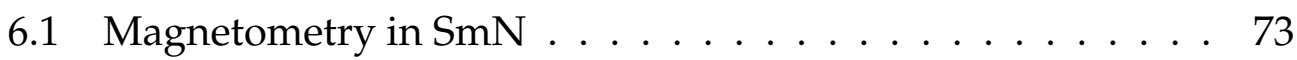

6.2 Ferromagnetic resonance in $\mathrm{SmN} \ldots \ldots . . . . . . .77$ 
$\begin{array}{lll}7 & \text { EuN preliminary investigation } & 81\end{array}$

7.1 Sample preparation ... . . . . . . . . . . . . . 82

7.2 Characterisation .................. 83

$\begin{array}{llr}8 & \text { Conclusion } & 87\end{array}$ 


\section{Acknowledgements}

While only one name appears on the front of this thesis, there has been an immense amount of support from colleagues, friends and family throughout the past twelve months that has contributed to the completion of this MSc.

I thank Senior Lecturer Ben Ruck, my supervisor, and Emeritus Professor Joe Trodahl, who have both provided guidance and wise counsel on experimental and conceptual issues throughout the project. For useful discussions and assistance with experiments I thank almost-Dr. Andrew Preston, Visiting Professor Claire Meyer, and Dr. Jan Richter, who made up the rest of the Rare-Earth Nitrides group at Victoria University during this project.

Samples were grown in collaboration with Ian Farrell and the group of Associate Professor Steve Durbin and Professor Roger Reeves at the University of Canterbury and I am grateful for their hard work.

Further thanks go to Jurgen von Bardeleben (CNRS Paris 6), and Laurent Ranno (Universite Joseph Fourier) for accommodating me in their labs and providing assistance with ferromagnetic resonance and transport measurements respectively. The RBS measurements by Peter Murmu and Dr. John Kennedy at GNS were greatly appreciated.

Final thanks go to Ms. Fiona Hodge, Hugo, Julian, Joanna and Courtenay Ludbrook, for keeping me fed, clothed, and entertained, and for the useful distractions from work.

This project was funded by a Victoria masters by thesis scholarship and an Ernest Marsden scholarship in experimental physics. A New Zealand study abroad award funded some of the measurements undertaken in France. 



\section{Chapter 1}

\section{Introduction}

The rare-earth nitrides (RENs) are a fascinating group of materials combining theoretical and experimental challenges with the promise of extensive technological applications. As the field of spintronics develops and the potential of harnessing electron spin in electronics approaches realisation, there is a growing need for materials that show a strong coupling between their magnetic and electronic properties $[1,2,3]$.

Spintronics, or spin-based electronics, is a growing field where the property of spin is used in electronic devices to "store, encode, access, process and/or transmit information," tasks that have traditionally been performed by the charge of the electron [2]. Spintronics relies on the creation and detection of spin-polarised currents which is usually done by passing a current through a metallic ferromagnet. Spin injection into a semiconductor, desirable for integration of spin-based devices with charge-based ones, is severely limited by the usual impedance mismatch of the two materials [4]. Research into materials that are intrinsically semiconducting as well as magnetic is therefore of significant importance. Currently, the main avenue of research to that end involves doping semiconductors with magnetic particles. So called dilute magnetic semiconductors (DMS) have a number of drawbacks that may limit their use. For instance, the magnetic dopants tend to act as electronic dopants too making it difficult to control the carrier concentration [5].

Members of the rare earth nitride series show a range of electronic and mag- 
netic properties that would be advantageous to the development of spintronic devices. A number of the series are ferromagnetic semiconductors, while others may be half-metals or metallic. Applications derived from exchange splitting of the bands have recently been demonstrated in $\mathrm{EuO}$, where it acts as an efficient electron spin filter [6]. The exchange splitting of the conduction bands in some of the rare earths is of a similar size to that of EuO, and Ref.[5] has pointed out that the splitting of the valence bands as well as the conduction bands may make the RENs even more suited to spin filter device applications.

The rare-earth metals (REs), also known as the lanthanides, are a group of metals unified by the progressive filling of their tightly bound $4 f$ shell. Electrons in the $4 f$ level are the origin of the magnetic properties, but do not participate directly in the bonding or transport in rare earth compounds; an interesting state of affairs. A surge of interest in the rare-earth pnictides (group 15 elements) and chalcogenides (group 16 elements) during the 1960's and 70's was driven by the prospect of ferromagnetic semiconductors [4]. These compounds allowed a large expanse of parameter space to be studied: for the same rare-earth cation, different anions modified the lattice parameter and the conductivity. The effect of the $4 f$ levels could be investigated by substituting one RE for another. All the while, the vast majority of these compounds, and all the RENs crystallised into the $\mathrm{NaCl}$ rock salt structure [7]. However, research into the bulk electronic and magnetic properties of the RENs was hampered by difficulties in the preparation of stoichiometric and oxygen free samples [8]. Partly due to these problems with sample preparation, the picture that emerged from these initial studies was far from clear. Confusion and conflicting results remained over the fundamental magnetic and electrical properties $[9,10]$.

The last fifteen years has seen a renewal of interest in these materials for three reasons. Firstly, sample fabrication methods have improved, making it possible to obtain relatively stoichiometric samples free of contaminants. Advances in theory and improvements in band structure calculations have predicted a range of interesting properties in these materials $[11,12,13,14,15,16]$. With the theoretical and experimental investigations achieving maturity, it has led to the prospect of REN devices, the third reason for the renewed interest in these materials.

Recent improvements in the growth of RENs has been led by a number of 
groups using different techniques. Luenberger et al. successfully grew $\mathrm{GdN}$ thin films using reactive ion beam sputtering and a nitrogen plasma [17]. Polycrystalline films were grown on silicon (Si), sapphire, kapton and mylar substrates, and capped for $e x$ situ studies with a $50 \mathrm{~nm} \mathrm{Al}$ or W layer. The group at Victoria University of Wellington led by Dres. Ben Ruck and Joe Trodahl has grown RENs by evaporating the rare earth element in the presence of a nitrogen background. GdN, SmN, EuN, DyN and ErN have all been produced in this manner on substrates of $\mathrm{Si}$, sapphire and yttria stabilised zirconia (YSZ). $50 \mathrm{~nm} \mathrm{GaN} \mathrm{capping}$ layers have been used to protect the films during ex situ studies. This relatively simple technique has also been used to grow samples in situ at synchrotrons, thus avoiding the complication of a capping layer.

In 2007 Gerlach et al. reported the growth of epitaxial GdN films on $\mathrm{MgO}$ substrates by molecular beam epitaxy. Films were shown to be of high crystalline quality but little was reported on the magnetic or transport properties [18]. More recently, epitaxial growth of GdN on GaN by MBE has been reported [19], an important step towards device fabrication.

GdN is by far the most studied of the rare earth nitrides, and the growth efforts detailed above are testament to that. Work has been done on $\mathrm{EuN}$ and $\mathrm{SmN}$, the other materials reported on in this work, but there has not been the same progress in film growth [20,21,22]. We will now review some of the properties of high quality GdN thin films, and the results of previous studies of EuN and SmN.

$\mathrm{GdN}$ crystallises in the $\mathrm{NaCl}$ rocksalt structure with a lattice parameter of $4.99 \AA \pm 0.02 \AA$. EuN and $\mathrm{SmN}$ take the same structure with lattice parameters of 5.00 and $5.04 \AA$ respectively. The three materials fit into the broader trend of the Lanthanide contraction, where the lattice parameter decreases across the rare earth series due to the decreasing size of the RE ion $[8,5]$.

Theoretical investigations of the band structure of $\mathrm{GdN}$ are dominated by the treatment of the $4 f$ levels. Early investigations treating the $4 f \mathrm{~s}$ as core states predicted a semiconducting groundstate with a bandgap of $1 \mathrm{eV}$ [23]. Recently, a range of predictions have been reported based on more advanced band structure calculation techniques using the local spin density approximation (LSDA) with various corrections. Aerts et al. report a half-metallic groundstate based on 


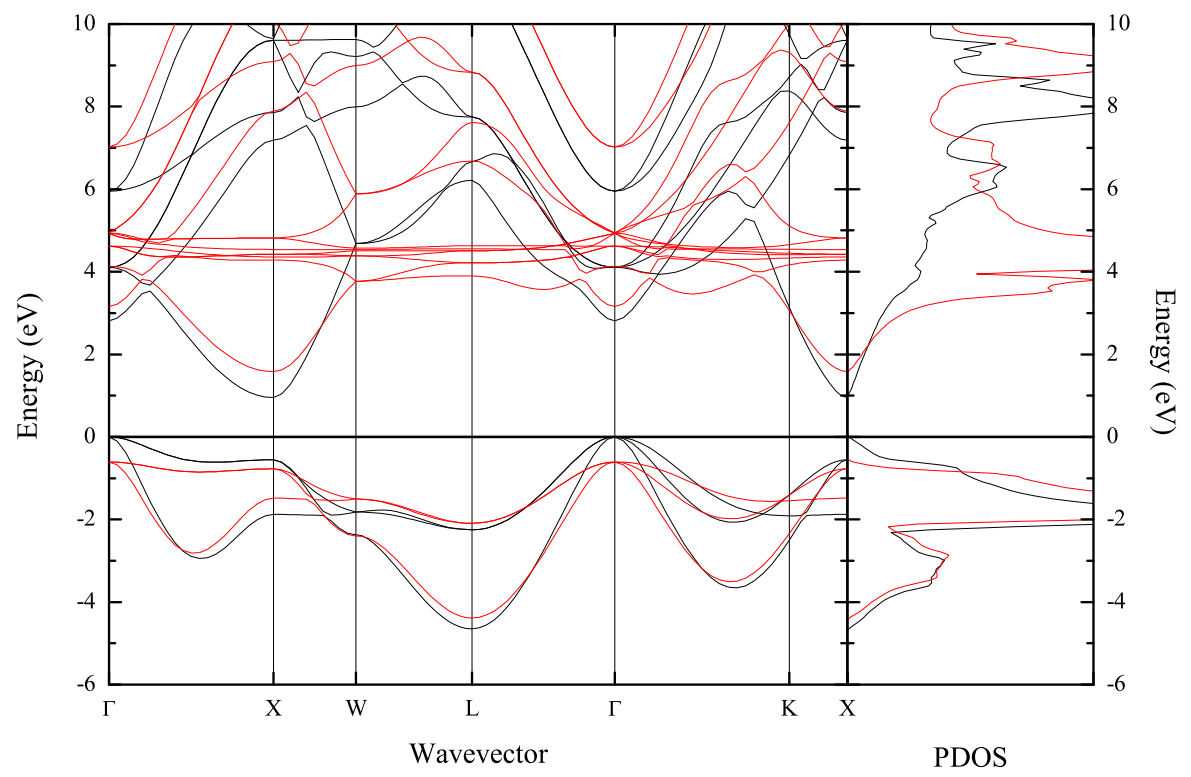

Figure 1.1: Spin resolved band structure for GdN based on LSDA+U calculations. Majority bands in black, minority bands in red.

LSDA-SIC calculations [14], while Duan et al. report a similar groundstate based on LSDA+U calculations [24]. Duan also demonstrates an interesting half-metal to semiconducting transition as a function of lattice parameter, implying the electronic and magnetic properties can be tuned by applying stress [5].

A series of papers by Lambrecht $e t$ al. predict a semiconducting groundstate. Initial calculations predicted a bandgap which was later tuned to reproduce experimental data $[12,11,13]$. An advanced calculation by Chantis et al. also finds a semiconducting groundstate [25]. The spin resolved band structure of GdN reported in Ref.[26] is reproduced in Figure 1.1. The majority (black) and minority (red) spin states are treated separately and are found at slightly different energies due to the exchange interactions with the occupied $4 f \mathrm{~s}$ in the ferromagnetic state, a feature known as exchange splitting. The occupied $4 f$ levels are off the scale but lie at about $-7 \mathrm{eV}$. The direct bandgap at the $\mathrm{X}$ point is $1.3 \mathrm{eV}$ in the paramagnetic state, and is found to shift to $0.9 \mathrm{eV}$ in the ferromagnetic state [27].

Band structure calculations for $\mathrm{SmN}$ and EuN predict half-metallic groundstates [14, 13]. EuN in particular is difficult to interpret as in one calcu- 
lation there was some doubt as to whether the divalent or trivalent solution was energetically more favourable.

The experimental evidence relating to the electronic groundstate of GdN strongly suggests that it is semiconducting. Temperature dependent resistivity measured from polycrystalline films show semiconducting behaviour[28] while optical measurements on similar films indicate a band gap of $1.3 \mathrm{eV}$ above the Curie temperature ( $\mathrm{T}_{C}$, the temperature below which the material exhibits ferromagnetic order) [27]. Both of these measurements are consistent with a narrowing of the bandgap at $\mathrm{T}_{C}$. This manifests itself as a drop in the resistivity below $\mathrm{T}_{C}$, while in optical measurements there is a change in the absorption onset edge. The bandgap in the ferromagnetic state was measured to be $0.9 \mathrm{eV}$. Transport measurements on polycrystalline films indicate that $\mathrm{SmN}$ is a semiconductor[20] in contrast with theoretical predictions, although no optical measurements on high quality films have been performed. No measurements have been reported on reliable EuN.

GdN is well established as ferromagnetic below $70 \mathrm{~K}[28,17,19]$. The magnetisation saturates at a value of $7 \mu_{B} / \mathrm{Gd}$ ion as expected for the pure spin moment arising from the 7 electrons in the $4 f$ shell. There is currently no theoretical model that predicts a Curie temperature in agreement with experimental values. The most detailed theoretical investigation of the magnetic properties of $\mathrm{GdN}$ to date predicts a $\mathrm{T}_{C}$ of just $11 \mathrm{~K}$ [29]. This reflects the difficulty of understanding and modelling the complex exchange interactions that lead to ferromagnetism in this material. This will be discussed in more detail in Chapter 3.

$\mathrm{SmN}$ is ferromagnetic with a Curie temperature of about $30 \mathrm{~K}[20,21]$. With a $4 f^{5}$ configuration, $\mathrm{Sm}^{3+}$ has spin and orbital moments that are expected to nearly cancel resulting in a very small moment per ion [13]. This has been observed in Ref.[21] in polycrystalline films on silicon. While near vanishing magnetic moments have been reported in $\mathrm{Sm}^{3+}$ compounds, this is the only semiconducting compound known to show this behaviour. EuN is similarly interesting although there is even less experimental data on it. $\mathrm{Eu}^{3+}$ is expected to have total angular momentum $J=0$ in its groundstate and is therefore non-magnetic as its moment will have no projection along an applied field. There could still be some long range magnetic ordering[22], and so experiments are needed to see how this material behaves magnetically. 
In light of the discrepancies between theory and experiment in $\mathrm{GdN}$, and the limited experimental and theoretical work done on $\mathrm{SmN}$ and $\mathrm{EuN}$, there is a clear need to make samples of high crystalline quality and low impurity densities. This will lead to a better understanding of the intrinsic properties of these materials which can then guide the theory, and perhaps lead to the incorporation of these materials in spintronics devices.

This work focuses on the growth and properties of GdN, SmN, and EuN. Chapter 2 is an introduction to some of the experimental techniques used to determine the structural, magnetic and electronic properties of the thin films grown by PLD. Chapter 3 presents a brief introduction to magnetism with an emphasis on the treatment of ferromagnetism in the rare earth nitrides. Chapter 4 begins with an introduction to pulsed laser deposition, and then goes on to detail the growth and structural characterisation of $\mathrm{GdN}$ and SmN. Chapter 5 contains measurement and analysis of the magnetic and transport properties of GdN films, where magnetometry, ferromagnetic resonance (FMR) and Hall effect are the key measurements performed. In Chapter 6, the magnetic properties of SmN using magnetometry and FMR are presented. Finally, Chapter 7 presents the growth and preliminary characterisation results of EuN. 


\section{Chapter 2}

\section{Experimental techniques}

A range of experimental techniques were used to characterise the samples and to explore the magnetic and transport properties of the materials. This chapter provides an introduction to some of these techniques and, where relevant, some of the underlying theory.

\subsection{Ferromagnetic resonance}

Ferromagnetic resonance (FMR) is a technique that probes the behaviour of electronic spin in both internal and external (applied) magnetic fields. In the absence of an internal magnetic field, the technique is known as electron paramagnetic resonance (EPR), or electron spin resonance (ESR). We will first look at EPR and then apply this to ferromagnetic materials.

In a paramagnetic material in the absence of an applied magnetic field, the magnetic moment of each paramagnetic element $\left(\mathrm{a} \mathrm{Gd}^{3+}\right.$ ion, say) is randomly oriented due to thermal fluctuations. This degeneracy is lifted by an applied magnetic field, which brings about a Zeeman splitting of the energy associated with the moment aligning parallel or anti-parallel to the applied field. The occupancy of the two levels is determined by Boltzmann statistics, resulting in the lower energy (spin aligned parallel to the field) having a larger population. It is transitions between these Zeeman split levels that are induced and then measured in EPR. The size of the splitting depends directly on the magnetic field, and when this is 


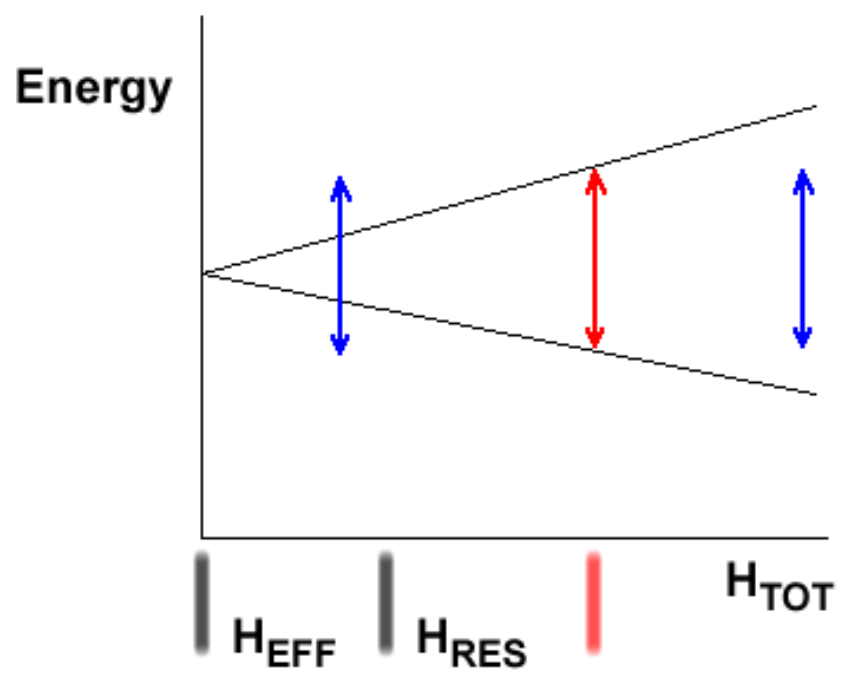

Figure 2.1: Illustration of the Zeeman splitting leading to an absorption of energy when the splitting is equal to the energy of the splitting. The difference from EPR is shown by the $\mathrm{H}_{\text {eff }}$ term which increases the total field causing the splitting.

equal to the energy of photons incident on the sample (in the microwave range), the population difference allows for a net absorption of energy, which forms the basis for the EPR signal.

Magnets found in a standard EPR or FMR setup typically range from 1 to 6 Tesla, and the incident photons are in the microwave range (typical microwave frequencies are $9 \mathrm{GHz}$ ( $\mathrm{X}$ band) or $34 \mathrm{GHz}$ ( $\mathrm{Q}$ band)). The sample is placed in a microwave cavity in a variable magnetic field. As the field is swept, there will be a strong absorption when the Zeeman splitting corresponds to the microwave energy, and this is detected as an energy loss in the cavity. The external field $\mathrm{H}_{0}$ is modulated by the addition of small oscillating field which improves the signal to noise ratio, and has the side effect that the detected signal is in fact the first 
derivative of the absorption.

FMR measurements differ from EPR measurements in a number of ways. The total field is no longer just the applied magnetic field, but includes the internal and demagnetising fields. This can shift the resonance by up to several kOe [30]. Another feature of FMR is very strong signals. The strength of the signal depends on the population difference between spin up and spin down electrons, and for a ferromagnetic material in its ground state, this difference is $100 \%$ leading to a very strong signal. Figure 2.1 illustrates the absorption of energy when the energy of the microwave radiation, shown as the height of the arrows, equals the Zeeman splitting. The applied field required for resonance is shown as $\mathrm{H}_{\mathrm{RES}}$. The actual field experienced by the magnetic ions has a contribution from the internal fields $\mathrm{H}_{\mathrm{EFF}}$, and as these contributions change, so too will the resonance field.

We will now discuss FMR with respect to thin films of ferromagnetic material, and the formalism used to analyse the data. Figure 2.2 shows the geometry for FMR measurements of this type. With the film in the x-z plane, $\theta$ and $\theta_{H}$ refer to the angles made by $\mathbf{M}$ and $\mathbf{H}$ with the $\mathbf{z}$-axis (the polar angle in spherical coordinates). Similarly, $\phi$ and $\phi_{H}$ refer to the angles made by $\mathbf{M}$ and $\mathbf{H}$ with the $x$-axis in the $x-y$ plane (the azimuthal angle). The aim is to describe the equilibrium orientation of the magnetisation $\mathbf{M}$ in the presence of a magnetic field $\mathbf{H}$ applied at different angles relative to the crystal axes. In the absence of any form of anisotropy the two would be colinear. When this is not the case, we can simulate the angular dependence of the equilibrium orientation of $\mathbf{M}$ and learn about the anisotropy of the film.

We begin with the Smit-Beljers resonance equation relating the resonance frequency to the free energy [31]:

$$
\frac{\omega}{\gamma}=\frac{1}{M \sin \theta}\left\{\frac{\partial^{2} F}{\partial \theta^{2}} \frac{\partial^{2} F}{\partial \phi^{2}}-\left[\frac{\partial^{2} F}{\partial \phi \partial \theta}\right]^{2}\right\}^{\frac{1}{2}}
$$

where $\omega$ is the resonant angular frequency, $\gamma$ is the gyromagnetic ratio, $\mathrm{F}$ is the magnetic free energy density and the double derivatives are taken with respect to the angles of magnetisation.

The magnetic free energy density is defined for the particular crystal structure 


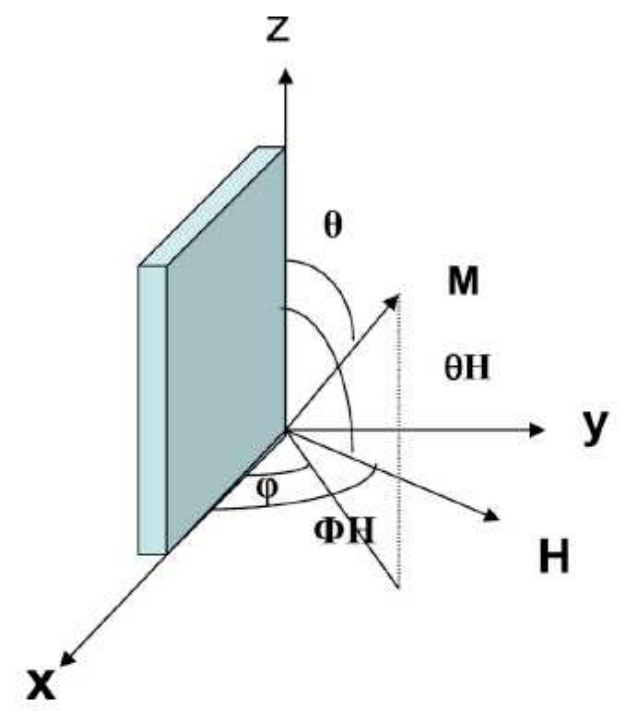

Figure 2.2: Geometry of the sample and applied field for FMR measurements

and geometry of the sample.

$$
F=-\mathbf{M} \cdot \mathbf{H}+K_{1}\left(\alpha_{1}^{2} \alpha_{2}^{2}+\alpha_{2}^{2} \alpha_{3}^{2}+\alpha_{3}^{2} \alpha_{1}^{2}\right)+\left(2 \pi M^{2}-K_{u}\right) \alpha_{2}^{2},
$$

where $\mathbf{M} . \mathbf{H}$ is the Zeeman energy, $\mathrm{K}_{1}$ is the $4^{\text {th }}$ order cubic anisotropy constant, $\alpha_{i}$ are the direction cosines of $\mathbf{M}$ relative to the cubic crystal axes, and $\mathbf{K}_{u}$ a second order uniaxial anisotropy term. The terms in this expression have been selected for a cubic material with a possible uniaxial anisotropy due to strain.

The static equilibrium condition is given by

$$
\frac{\partial F}{\partial \theta}=\frac{\partial F}{\partial \phi}=0
$$

We restrict ourselves to the case where the $\mathbf{H}$ is applied in the $\mathbf{x - y}$ plane so the static equilibrium condition of the magnetisation is satisfied for $\theta=\pi / 2$ and

$$
H \sin \left(\phi_{H}-\phi\right)=4 \pi M_{\mathrm{eff}} \sin \phi \cos \phi+\frac{K_{1}}{2 M} \sin 4 \phi
$$

with $4 \pi M_{\text {eff }}=4 \pi M-2 \frac{K_{u}}{M}$. 
The resonance frequency can now be found from the second derivatives of $F$ using expression 2.1 giving

$$
\begin{array}{r}
\left(\frac{\omega}{\gamma}\right)^{2}=\left[H \cos \left(\phi_{H}-\phi\right)-4 \pi M_{e f f} \sin ^{2} \phi+\left(\frac{K_{1}}{M}\right)\left(2-\sin ^{2} 2 \phi\right)\right] \times \\
{\left[H \cos \left(\phi_{H}-\phi\right)+4 \pi M_{e f f} \cos 2 \phi+\left(\frac{2 K_{1}}{M}\right) \cos 4 \phi\right] .}
\end{array}
$$

Equations 2.5 and 2.4, with $\mathrm{K}_{1}$ and $\mathrm{K}_{u}$ known, completely describe the magnetisation of the sample and the resonance conditions for the orientations of $\mathbf{H}$ relative to the film plane.

Simulating an FMR angular dependency involves determining values of the anisotropy constants, and then using these to simulate the resonance field as a function of the orientation of the applied field. From equation 2.4 we see that $\mathbf{H}$ and $\mathbf{M}$ are only colinear for $\phi=0$ and $\phi=\pi / 2$, that is, when $\mathbf{H}$ is in the film plane or perpendicular to it. Using these equilibrium state values for $\phi$ (with $\phi_{H}=\phi$ ), and experimental values for the resonance field and the magnetisation $(\mathrm{H}$ and $\mathrm{M})$ we have two equations that can be solved simultaneously for $\mathrm{K}_{1}$ and $\mathrm{K}_{u}$. Using these values of $K$, we now look for the static equilibrium conditions of $\mathbf{M}$ as $\mathbf{H}$ is varied; equations 2.4 and 2.5 are solved simultaneously for $\mathrm{H}_{r e s}$ and $\phi$. The resulting angular dependence of $\mathrm{H}_{r e s}$ with $\phi_{H}$ is compared to the experimental data in order to determine the validity of the anisotropy constants used.

\subsection{Magnetometry}

Thin films of magnetic material require high sensitivity magnetometers for measuring magnetic moments. A Quantum Designs Magnetic Property Measurement System was used to measure both temperature dependent and field dependent magnetisation. This device has three principle features. First, it uses a superconducting quantum interference device (SQUID) to measure very small fields. This makes it useful for very small samples, or samples with small magnetic fields. Secondly, it uses superconducting coils to produce large fields up to 6 Tesla. Finally, the magnetisation can be thoroughly studied at a range of temperatures, from $2 \mathrm{~K}$ up to $300 \mathrm{~K}$. 
(a)

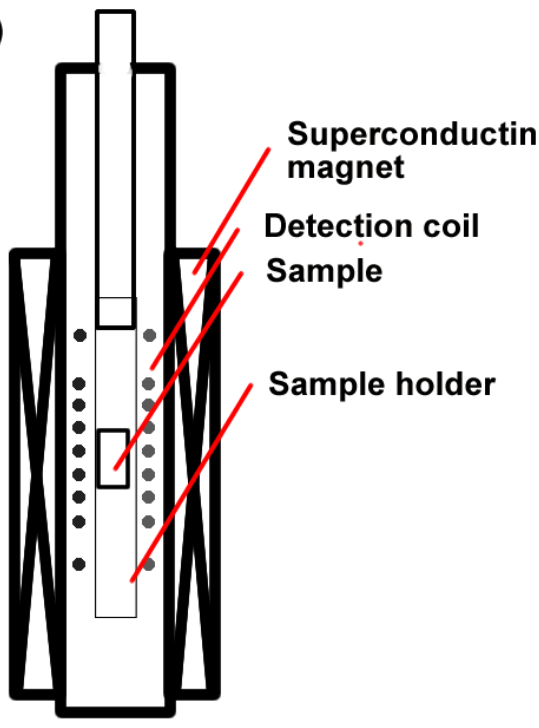

(b)

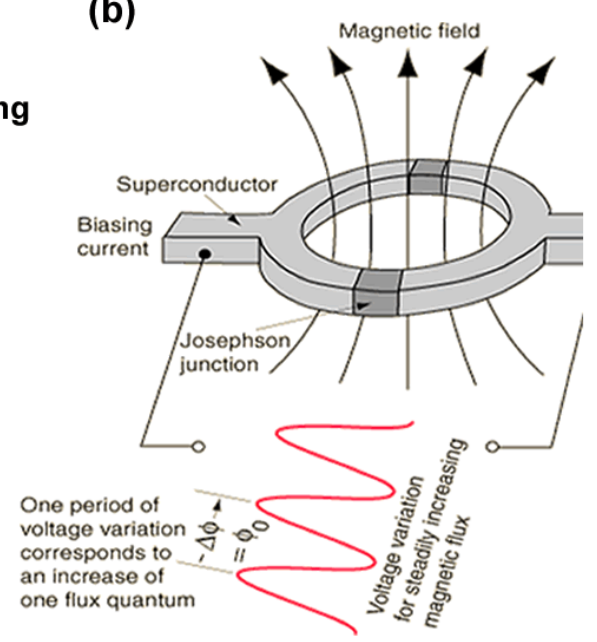

Figure 2.3: The main components of a SQUID magnetometer.

The sample is moved in the applied DC magnetic field and the change in flux enclosed in the superconducting pickup coil is inductively coupled to the SQUID. Changes in the flux are detected by changes in the voltage across the Josephson junction, the main component of the SQUID [32]. Samples are mounted in a nonmagnetic plastic straw in order to minimise any stray signals. It is important that the region around the sample is homogenous as any inhomogeneity may contribute a signal to the highly sensitive measurement. The size of the straw limits the size of the sample, and so these are typically cleaved to approximately $3 \mathrm{~mm} \times 5 \mathrm{~mm}$.

The measurement process is largely automated and involves measuring the total moment of the sample at different temperatures and applied fields. The two key measurement processes are detailed below:

Zero field cooled and field cooled An applied field will not always evoke the same magnetic response from a material at a given temperature. It will depend on whether the material has been cooled with a magnetic field applied, or 
without. A ZFC measurement proceeds by cooling the sample from above its Curie temperature to $2 \mathrm{~K}$, applying some magnetic field, and measuring the magnetic moment as the sample is heated. For a FC measurement, the field is applied while the sample is at a temperature above $\mathrm{T}_{C}$ and the sample is then cooled to $2 \mathrm{~K}$, measuring the magnetisation as it cools. If the applied field is large enough, both FC and ZFC samples can be completely magnetised (all domains arranged colinearly) at all temperatures, and the curves will be indistinguishable.

Hysteresis loops At a given temperature, the applied field can be swept up to a maximum field (typically $6 \mathrm{~T}$ ), down to a maximum field in the opposite direction, and back. This tells us, among other things, the degree to which the sample remains magnetised when the applied field is reduced to zero (the remnance), and how easily the magnetisation of the sample can be reversed (the coercive field).

The total magnetic moment is measured by the SQUID in 'emu' from the CGS system of units. Magnetisation, or moment per unit volume, is often quoted in units of emu $/ \mathrm{cm}^{3}$. We also use units of Bohr magnetons per magnetic ion ( $\mu_{B} /$ ion). The Bohr magneton is approximately the magnetic dipole moment of a single electron; these units are convenient to compare with the number of electrons contributing to the magnetic moment. Both units require knowledge of the volume of the sample, which is the largest source of uncertainty in the results. The conversion between the units is straight forward.

\subsection{Transport, Hall and magnetoresistance mea- surements}

Transport properties were determined with four terminal measurements. This, along with a magnetic field that could be applied in-plane or out-of-plane allowed for measurements of the resistivity, Hall effect, and magnetoresistance. The temperature could be controlled between $2 \mathrm{~K}$ and $300 \mathrm{~K}$, and a magnetic field of up to $\pm 5 \mathrm{~T}$ could be obtained.

Resistivity measurements could be taken as a function of temperature or as a 
function of applied field in- or out-of- plane. Magnetoresistance (MR) is quoted as the percentage change of the resistivity at high field $(4 \mathrm{~T})$ from the zero field (ZF) value:

$$
\% M R=100 \frac{\rho(4 T)-\rho(Z F)}{\rho(Z F)} .
$$

The Hall resistance is measured by running a current diagonally across the sample, and measuring the voltage perpendicular to this, while a magnetic field is applied perpendicular to the plane of the film. In an ordinary (non-magnetic) conductor in this geometry, the moving charge carriers experience a Lorentz force in the sample plane, perpendicular to their velocity. This leads to a build-up of charge until, at equilibrium, the transverse electric field is sufficient to oppose any further charge build-up. This transverse voltage is measurable and is known as the Hall voltage. Dividing by the current through the sample and normalising for the length and cross section of the sample, we define the Hall resistivity and it can be shown that

$$
\rho_{H}=R_{o} H
$$

where $\mathrm{H}$ is the magnetic field and $\mathrm{R}_{o}$ is the ordinary Hall coefficient, inversely proportional to the carrier concentration. Measuring the slope of the Hall resistivity with applied field allows determination of both the carrier concentration and the sign of the charge carriers. In a ferromagnetic material the situation is complicated by the magnetisation of the sample. There is an additional term proportional to the magnetization, $\mathrm{M}$, in the Hall resistivity, which can now be written [33]:

$$
\rho_{H}=R_{o} H+\mu_{0} R_{s} M .
$$

Here, $\mathrm{R}_{s}$ is the anomalous hall coefficient. The appearance of the anomalous Hall effect (AHE) is important evidence for intrinsic ferromagnetism[34] and from the above equation we can see that at zero field $(\mathrm{H}=0) \rho_{H}$ is proportional to the magnetisation of the sample. In order to extract the carrier concentration in a FM material, $\mathrm{M}$ must be saturated so that $\rho_{H}=R_{o} H+c$ where $\mathrm{c}$ is a constant. 
(a)

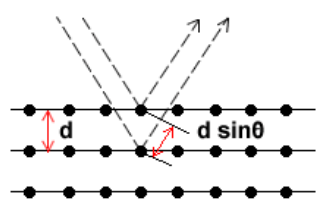

(b)

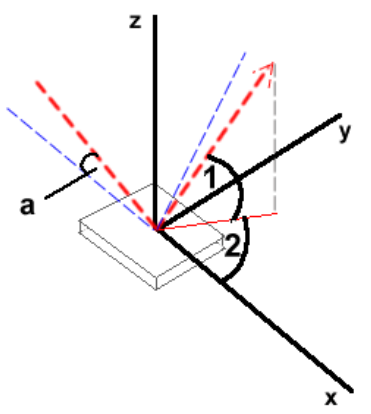

Figure 2.4: (a) Bragg condition for XRD. (b) Illustration showing the geometry of rocking curves and $\phi$ scans. Angle 1 is $\theta$, angle 2 is $\phi$, and angle ' $a$ ' relates to the rocking curve with $\omega=\theta+a$.

\subsection{X-ray diffraction}

X-ray diffraction (XRD) measures the constructive interference of $x$-rays reflecting from coherent lattice planes. Monochromatic radiation in the $\mathrm{x}$-ray wavelength range is incident on the sample at an angle $\theta$ to the surface. A detector is located also at an angle $\theta$ to the surface plane and measures a strong reflection when the radiation diffracted from different atomic planes interferes constructively. As the angle $\theta$ is continuously varied (known as a $\theta-2 \theta$ scan) the detected signal changes in intensity, with peaks at values of $2 \theta$ satisfying the Bragg condition

$$
2 d \sin \theta=n \lambda,
$$

where $d$ is the lattice plane spacing, $\lambda$ is the $\mathrm{x}$-ray wavelength, and $n$ is the order of the diffracted peak.

Rocking curves involve fixing the source and detector at some $\theta-2 \theta$ corresponding to a particular reflection and then, with the source and detector locked together so that the angle between them does not change, varying $\theta$ by $\pm a$, shown as the blue dotted line in Fig. 2.4(b). This varies the $c$ axis probed by the x-rays about the film plane normal, and gives information about the distribution of $c$ axes, or mosaic spread, in the film. So called ' $\phi$ scans' involve rotating the sample about its normal and taking rocking curves for various angles. 


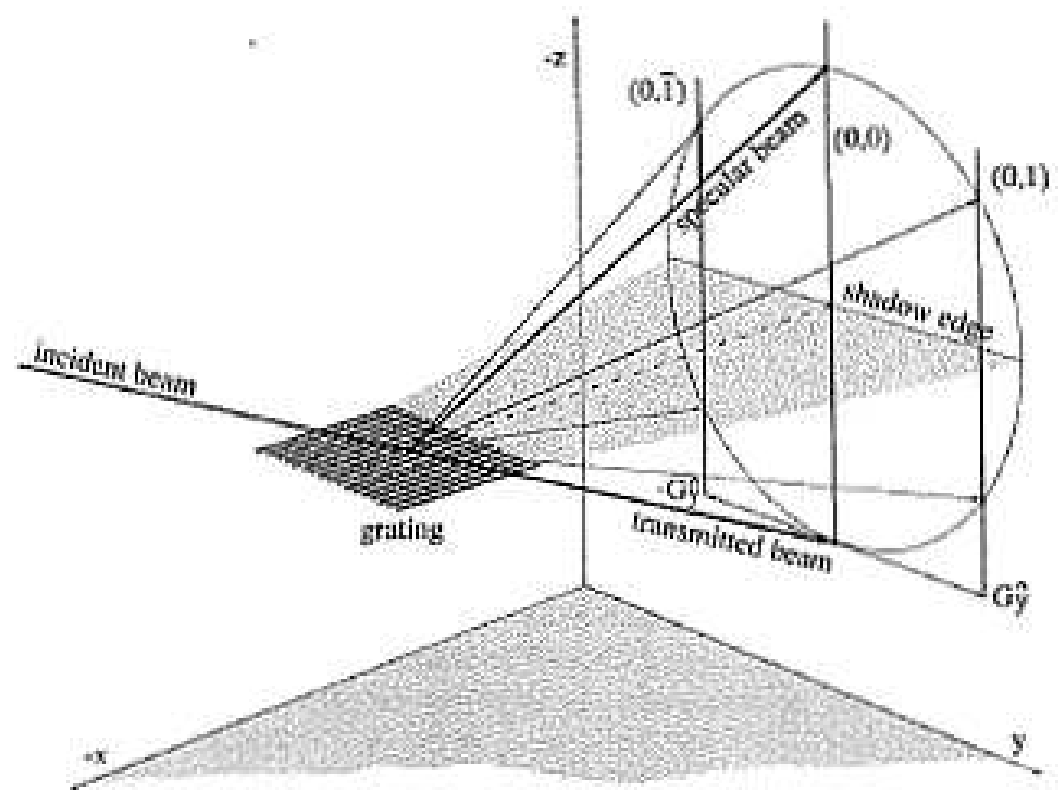

Figure 2.5: Schematic diagram of RHEED and the formation of streaks from a surface that acts as a diffraction grating [35].

\subsection{Reflection high energy electron diffraction}

The surface of the films were monitored in real time in situ using a Staib electron gun and KSA imaging camera with associated software. In reflection high energy electron diffraction, a beam of high energy electrons interact with the film surface at grazing incidence and are detected on a phosphorous screen. The electrons are at an energy of $20 \mathrm{keV}$, and the beam intensity is approximately $1.4 \mu \mathrm{A}$, or $10^{13}$ electrons per second. At grazing incidence, the electrons spend no more than $10^{-14} \mathrm{~s}$ interacting with the lattice and the collision is considered elastic [35]. It is only the top few monolayers that are involved in the diffraction process making this a truly surface sensitive technique.

The set up is shown in Figure 2.5. The well ordered structure on the surface acts like a 2D diffraction grating. The resulting diffraction pattern can be well understood in terms of the Ewald sphere in reciprocal space [35, 36]. Electrons may 
only take momentum from the crystal lattice in certain directions and in certain amounts, which depend on the reciprocal space lattice structure. This produces the characteristic patterns that may be associated with particular crystal structures. A 2D grid of atoms will provide momentum in directions perpendicular to the lines of reciprocal lattice points and hence a well ordered cubic material will exhibit sharp streaks with a spacing inversely proportional to the real space lattice spacing. The crystal structure and the in-plane surface lattice parameter may be extracted from the pattern. The width of the streaks also contain information about the coherence length on the surface. Mosaicity and island growth both contribute to the linewidth [35]. If the surface is rough, the electron beam will pass through the 'hills' and diffract. The effect on the pattern is large spots within the streaks.

The diffraction patterns were recorded using a KSA software package. From this, intensity profiles could be extracted for analysis. A region of the pattern 30 pixels high and as wide as necessary to capture all the streaks was selected accross the centre of the image. Integrating over the 30 pixels resulted in a line profile of the intensity as a function of horizontal pixel. The height of the region selected was kept the same for all growth analysis, and 30 pixels was found to optimise both the narrowness of the peaks and the smoothness of line in the line profile. The line profile could then be fitted with a custom Matlab program using lorentzian lineshapes for the peaks, plus a gaussian and linear background. Subtracting the background signal is important as peaks on a sloped background signal will appear shifted up the background gradient. Each captured RHEED image was analysed in this way, providing data on the peak positions, intensities and widths as a function of growth time. 



\section{Chapter 3}

\section{Introduction to the magnetism of the rare-earth nitrides}

The origin of magnetism in materials and the measurable manifestation of this magnetism are often two quite different things. The former relates to the quantum mechanics of many body systems, while the latter is governed by crystal structure, shape and quality. This thesis deals primarily with the latter, but this chapter provides an introduction to both aspects of magnetism.

Magnetism has its origins in the angular momentum of electrons. A free electron has an intrinsic angular momentum known as spin, a measurement of which will take a value of $\pm \frac{1}{2} \hbar$, or equally, spin up and spin down, defined relative to an applied magnetic field. If we put this electron in an atom then, loosely speaking, it orbits around the nucleus with some orbital angular momentum. These two angular momenta, labelled $S$ and $L$, are quantum numbers. Electrons in an atom or solid are characterised by their quantum numbers, and no two electrons can share the same quantum numbers in the same system. Within an atomic shell of total orbital moment $\mathrm{L}$, there are $(2 L+1)$ levels or orbitals. These orbitals are labelled by $m_{l}$, running from $-L$ to $L$ in integer steps. Each orbital can be occupied by two electrons with opposing spin, making the total number of sites in a shell equal to $2(2 L+1)$. In the $4 f$ shell for example, $L=3$, so the orbitals are labelled by $\mathrm{m}_{L}=-3,-2, \ldots 2,3$, and up to 14 electrons can be accommodated.

The magnetic moment of an electron or atom is related to its angular momen- 
tum by

$$
\mu=-g \mu_{B} \mathbf{X}
$$

where $g$ is known as a $g$-factor and $\mathbf{X}$ is an angular momentum, which could be the spin, $S$, the orbital angular momentum, $L$, or the total angular momentum, $J=L+S$. The electron spin $g$-factor is approximately equal to 2 , while the electron orbital $g$-factor is equal to 1 . The $g$-factor for the total moment $J$ is the Landé $g$ factor and is given by the well known result from quantum mechanics[37] $g=$ $\frac{3}{2}+\frac{1}{2}\left[\frac{S(S+1)-L(L+1)}{J(J+1)}\right]$.

The way that the electrons fill up the orbitals in the atom is very important. The lowest energy shells will be filled first, and filled shells have no magnetic moment: there are equal numbers of spin up and spin down electrons, and just as many orbit in one direction as the other, eliminating any net spin or orbital moment. The groundstate is populated according to a set of rules known as Hund's rules $[36,37]$ and it is when we get to a partially filled shell that the magnetic behaviour becomes interesting. The first of these rules is similar in origin to the exchange interaction leading to ferromagnetism and states that the total spin moment, the sum of all the individual spin moments, will be maximised to the extent allowed by the exclusion principle. Just like people filling up seats on a bus, electrons of the same spin polarisation will occupy separate orbitals until all orbitals are full, and only then will an electron of opposite spin share an orbital. The second rule states that orbitals are filled in order to maximise $\mathrm{L}$, to the extent consistent with the first rule. Intuitively, filling separate orbitals allows electrons to minimise their Coulomb energy. The final rule is due to the spin-orbit coupling and relates $L$ and $S$ to the total angular momentum, stating that $J=|L-S|$ when a shell is less than half full, and $J=L+S$ when a shell is more than half full. The total angular momentum $J$ is $(2 J+1)$-fold degenerate in the absence of a magnetic field. It can take a number of projections, $m_{J}$, along an applied field, with values ranging between $-J$ and $J$ in integer steps.

In the case where the groundstate J value (determined by Hund's rules) is the only thermally populated level, the interaction of an applied field with the atomic moment lifts the degeneracy of $J$, resulting in $2 J+1$ levels split by an energy proportional to the applied field. The lower levels (corresponding to the moment aligning with the field) are slightly more populated than the higher ones 
(moment aligning antiparallel with the applied field), leading to a net magnetisation parallel with the applied field, proportional to $1 / \mathrm{T}$. This leads to Curie's law:

$$
\begin{gathered}
\chi=\frac{N}{3 V} \frac{\mu_{B}^{2} \mu_{\mathrm{eff}}^{2}}{k_{B} T}=\frac{C}{T}, \\
\mu_{\mathrm{eff}}=g[J(J+1)]^{2},
\end{gathered}
$$

where $\mathrm{N}$ is the number of magnetic ions in volume $\mathrm{V}, \mu_{\text {eff }}$ is the effective number of Bohr magnetons per ion, $\mathrm{g}$ is the Landé factor, $\mathrm{J}$ is the total angular momentum, and $\mathrm{C}$ is the Curie constant. This 'law' applies over a wide range of temperatures and fields, but does break down at low temperatures $(\approx 1 \mathrm{~K})$ and high fields $(\approx$ $1 \mathrm{~T}$ ), where the magnetisation begins to saturate.

A second important case is when there are closely spaced $J$ multiplets (separated by an energy gap on the order of $k_{B} T$ ) such that it is not only the groundstate that is thermally populated. It can be shown that this leads to a constant, temperature independent term, inversely proportional to the $J$-spacing $[37,36]$. Curie's law is simply modified to

$$
\chi=\chi_{0}+C / T,
$$

where $\chi_{0}$ is the van Vleck paramagnetic susceptibility.

The treatment thus far deals only with materials where there is no interaction between the magnetic moments. Each atomic moment can be treated separately, and the susceptibility is found by summing over all the moments. Magnetic ordering involves the alignment of these atomic moments and can take a number of forms: ferromagnetism (FM) describes the case where all of the moments are aligned parallel. Antiferromagnetism (AFM) is the case where the atomic moments are arranged so as to give no total magnetic moment. There are numerous other more complex arrangements, but it is ferromagnetism that is of greatest interest here.

In the case of a ferromagnetic material (discussed below) where the susceptibility diverges at the Curie temperature, the paramagnetic behaviour is described by the Curie-Weiss law:

$$
\chi=\frac{C}{T-T_{C}} .
$$


More than a simple modification to Curie's law, it is a result that comes from 'mean field theory' (MFT) which treats the interactions acting on a particle as an average field from all the surrounding moments. MFT is widely used to interpret and understand magnetism but it is not without its shortcomings and a number of authors recommend against its uncritical usage [37, 38].

In ferromagnetic materials the moments order spontaneously (without any applied field) below a certain temperature known as the Curie temperature. Individual magnetic ions are somehow aware of the orientation of their neighbours and the interaction responsible for this can be broadly termed 'exchange'. We consider the wavefunction of neighbouring magnetic ions. Magnetism is inextricably linked to spin, and yet the Hamiltonian is spin-independent. The lowest energy state is determined based solely on the Coulomb repulsion, and this determines the symmetry of the spatial part of the wavefunction. This then imposes a symmetry on the spin part of the wavefunction, and this determines whether the groundstate is magnetic or not. In the simplified case of a two electron system, exchange leads to an energy difference between the spins aligning parallel or antiparallel. This is direct exchange, where the wavefunctions of the two electrons or magnetic ions overlap. There are also various indirect-exchange mechanisms such as 'superexchange', where the magnetic ions are separated by nonmagnetic ones; the exchange is mediated by the nearest or next-nearest neighbour. Ruderman-Kittel-Kasuya-Yoshida (RKKY) is a mechanism whereby the exchange is mediated by the conduction electrons and varies between ferro- and antiferromagnetic as a function of the spacing of the magnetic ions and the carrier concentration. Exchange in magnetically ordered materials can be due to a range of, often competing, mechanisms. Whatever the underlying cause, the exchange interactions can often be phenomenologically described by the following $[39,36]$ :

$$
H=-\frac{1}{2} \sum_{i j} J_{i j} \vec{S}_{i} \cdot \vec{S}_{j}
$$

This is the Heisenberg Hamiltonian, where $\vec{S}$ is a localised spin moment and $J$ is some exchange constant, and the sum runs over nearest neighbours. This is often used as the starting point of theoretical investigations [29, 24].

We will now look at two such detailed theoretical investigations of the mag- 
netic properties of GdN to gain an understanding of the exchange interactions. Duan et al. begin from a band structure calculation which finds GdN to be half metallic [24]. Calculating the energy difference between various possible spin structures (different AFM configurations and FM) the exchange parameters $J$ can then be calculated for the interactions between nearest and next nearest neighbours. $\mathrm{T}_{C}$ is calculated from the exchange parameters to be $38 \mathrm{~K}$ within the MFT framework. The exchange parameters are then interpreted in terms of well known exchange interactions. These authors suggest that there is a competition between a RKKY interaction coupling the $4 f s$ through the conduction electrons, and an antiferromagnetic superexchange where the $\mathrm{Gd} d$ electrons are coupled through the anion $p$ orbitals, and the Gd $d$ electrons are coupled to the $4 f$ s. The strength of the RKKY exchange is enhanced due to the starting point of an incorrect half-metallic band structure. GdN is in fact a semiconductor and so it is not clear that the conclusions drawn by these authors are correct.

Mitra and Lambrecht point out that in pure semiconducting GdN the ferromagnetic exchange cannot come from the RKKY interaction as there would be no conduction electrons [29]. Using a band structure tweaked to agree with experimental results they calculate exchange parameters $J_{1}$ and $J_{2}$ corresponding to nearest and next nearest neighbour interactions respectively. Like Duan et al. they relate $J_{2}$ to an antiferromagnetic superexchange, but for $J_{1}$ they propose a very different explanation. From the band structure, the exchange splitting at the top of the valence band and the bottom of the conduction band is reversed such that the majority spin bands are closer in energy, and are therefore expected to hybridize more strongly. This is found to induce equal and opposite Gd $5 d$ and N $2 p$ moments, with the Gd $5 d$ moment parallel with that of the Gd $4 f$. The authors propose that $\mathrm{GdN}$ is an 'antiferromagnet in disguise' with the small $\mathrm{Gd} d$ and $\mathrm{N} p$ moments locked in an antiferromagnetic arrangement, but with the Gd $d$ moment coupled to the much stronger Gd $f$ moment. All of this is contained in the exchange parameter $J_{1}$, from which a Curie temperature of $11 \mathrm{~K}$ is determined. This is in stark disagreement with experiment, motivating the need for more work on both the theory and experimental fronts.

We now move away from the microscopic origins and models of magnetism and look at some of the manifestations of magnetism. While all the moments in 
a ferromagnet may be ordered, the material may not exhibit a net moment. Domains may form within which all the moments are aligned, but the domains are randomly oriented resulting in an overall cancellation of moments. Only by applying a magnetic field can we align the domains, and when all the domains are similarly oriented, or the sample is a single domain, the magnetisation is saturated. Much information can be gained by study of the behaviour of the magnetisation as a function of applied field or temperature. Some quantities of interest are the remnance, the magnetisation that remains when an applied field is reduced to zero, and the coercive field which is the field required to return the magnetisation to zero after it has been saturated by a large field applied in the opposite direction. These quantities are closely related to the anisotropies of the material.

Within a single magnetic domain the orientation of the magnetisation is coupled to the crystal lattice through the spin-orbit coupling and the crystal field, and thus not all crystallographic axes are equal [38, 40]. Magneto-crystalline anisotropy leads to easy and hard axes of magnetisation within a domain where the anisotropy relates to the energy required to move the magnetisation from an easy axis to a hard axis. The origin of magneto-crystalline anisotropy can be understood in terms of the shape of the electronic orbitals, which couple to the lattice [40, 41].

The shape of a domain also plays a role in defining the direction of magnetisation via the long range magnetic dipole interaction. The shape effect can be described by an anisotropic demagnetising field $\mathbf{H}_{d}$ described by $\mathbf{H}_{d}=-\mathfrak{N M}$ where $\mathfrak{N}$ is the demagnetising tensor which depends on the shape $[40,38]$. In the case of a thin film, only elements perpendicular to the surface are non-zero, and $\mathfrak{N}_{\perp}=1$. The demagnetising field makes the sample more difficult to magnetise out of plane, and for a field applied out of plane, it is shown that $H_{d}=-4 \pi M$ [39]. 


\section{Chapter 4}

\section{Sample preparation}

The films investigated in this study were grown primarily by pulsed laser deposition (PLD). Previously in our group at Victoria University of Wellington, rare earth nitrides have been grown by thermal evaporation deposition (TED) at or near room temperature and so the move to PLD reflects a novel avenue of research. Good descriptions of the TED system exist elsewhere and so will not be given here [42], however the reasons for looking to a new growth method are important. The main advantage of the PLD system is the heated substrate stage, allowing us to grow at temperatures of up to $900^{\circ} \mathrm{C}$. The chamber is also equipped with a RHEED system. It was expected that elevated growth temperatures would lead to higher crystal quality and perhaps better nitrogen incorporation, while the RHEED would give valuable information on the crystal structure. Of course the growth dynamics of PLD will be quite different to those of other methods and so in this chapter we look at the method of pulsed laser deposition (PLD), and the structural characterisation of the samples grown by this method.

\subsection{Pulsed laser deposition}

\subsubsection{Introduction to pulsed laser deposition}

In pulsed laser deposition (PLD) material is ablated from a target by an incident laser. In the present work, the laser arrives at the target in $25 \mathrm{~ns}$ pulses, with a fre- 
quency on the order of $10 \mathrm{~Hz}$. Following the analysis of Ref. [43] we can describe the process in three steps: laser-target interaction, laser-plasma interaction, and plasma expansion. In the first stage, the laser energy is absorbed by the target. For the metallic targets used in this work, the skin depth (characteristic absorption length of the laser) is much smaller than the thermal diffusion length (the distance the heat diffuses on the timescale of the laser pulse). Thus the amount of target material evaporated is determined by the thermal diffusion length, which is on the order of $1 \mu \mathrm{m}$. In the second stage, a plasma forms due to the interaction of the evaporated material and the laser, and this continues to strongly absorb the laser energy. The plasma undergoes an isothermal expansion, with the expansion velocity greatest in the direction of smallest plasma dimension; the plasma expands primarily in the direction normal to the target surface.

These first two steps happen on the time-scale of a single laser pulse. The third step, the adiabatic expansion of the plasma, happens after the laser pulse. Once again it is found that the highest velocities are in the direction of smallest dimension of the plasma. This gives rise to the characteristic plume shape of the ablated material. One effect of this preferential acceleration is that there is an angular dependence of the flux of particles. This can cause problems for the uniformity of films. Thickness variations across deposited films have been reported to show $\cos ^{(5-12)}(\theta)$ variations $[44,43]$. This would put an upper limit of about $5 \%$ on the variation of the thickness across a $10 \mathrm{~mm}$ wide film due to this particular effect.

Another problem for the creation of uniform thin films is the presence of droplets or nanoclusters. "Splashing" can occur when small high pressure regions appear near the molten surface of the target [45], sending particulates out with enough energy to reach the substrate. The presence of droplets on the substrate has also been attributed to target roughness [46]. Droplets may be minimised by altering the laser spot area and fluence [44].

\subsubsection{The PLD system}

For the present study, the system consists of an ultra high vacuum (UHV) chamber with a base pressure of $5 \times 10^{-9}$ mbar. There is a laser port through which a $248 \mathrm{~nm} \mathrm{KrF}$ excimer laser is focused onto an ablation target. The targets used 
were metallic disks, 2 inches in diameter and 1/4 inches thick, purchased from Kurt J. Leskar company. The targets were $99.9 \%$ pure with the main impurities being other rare earths, reflecting the difficulty of separating these elements from one another. YSZ caps were ablated from a large YSZ single crystal cleaved to the approximate dimensions. Targets were rotated at $2 \mathrm{~Hz}$ so that the laser would hit a fresh spot with each pulse.

Directly above the target at a distance of approximately $6 \mathrm{~cm}$ sit the substrates. These are secured to a molybdenum plate with molten indium. The positioning of the substrates with respect to the laser spot on the target is chosen to attempt to maximise the atomic flux while minimising the particulate flux. The presence of particulates is one of the drawbacks of PLD that will be discussed below. For the majority of growths, the primary substrate was located slightly off centre from directly above the laser spot. The primary substrate is designated by the fact that we were monitoring the RHEED pattern from this sample during and after growth. Any secondary substrates were loaded so as to be as close to the primary one as possible, but without interfering with the RHEED.

$99.999 \%$ pure nitrogen enters the growth chamber by way of a Oxford Applied Research RF plasma source. Both the plasma power and the nitrogen partial pressure are important variables in these growths. Ion deflection plates were consistently used so that only atomic and excited nitrogen were present.

The laser pulses are $25 \mathrm{~ns}$ in duration, and occur at a frequency of $10 \mathrm{~Hz}{ }^{1}$ Laser fluence is the energy per unit area at the surface of the target, and this quantity has a significant effect on the ablation of the target. The energy of the laser can be easily adjusted, while the spot area can be varied by changing the distance between lens and target.

\subsubsection{PLD samples}

From the description of the PLD system given above, we have introduced a number of variable growth parameters. We will now look at the samples grown by this method, and how the variation of some of these parameters affects the crys-

\footnotetext{
${ }^{1}$ While the frequency is adjustable, it was not changed for the duration of the work reported here.
} 


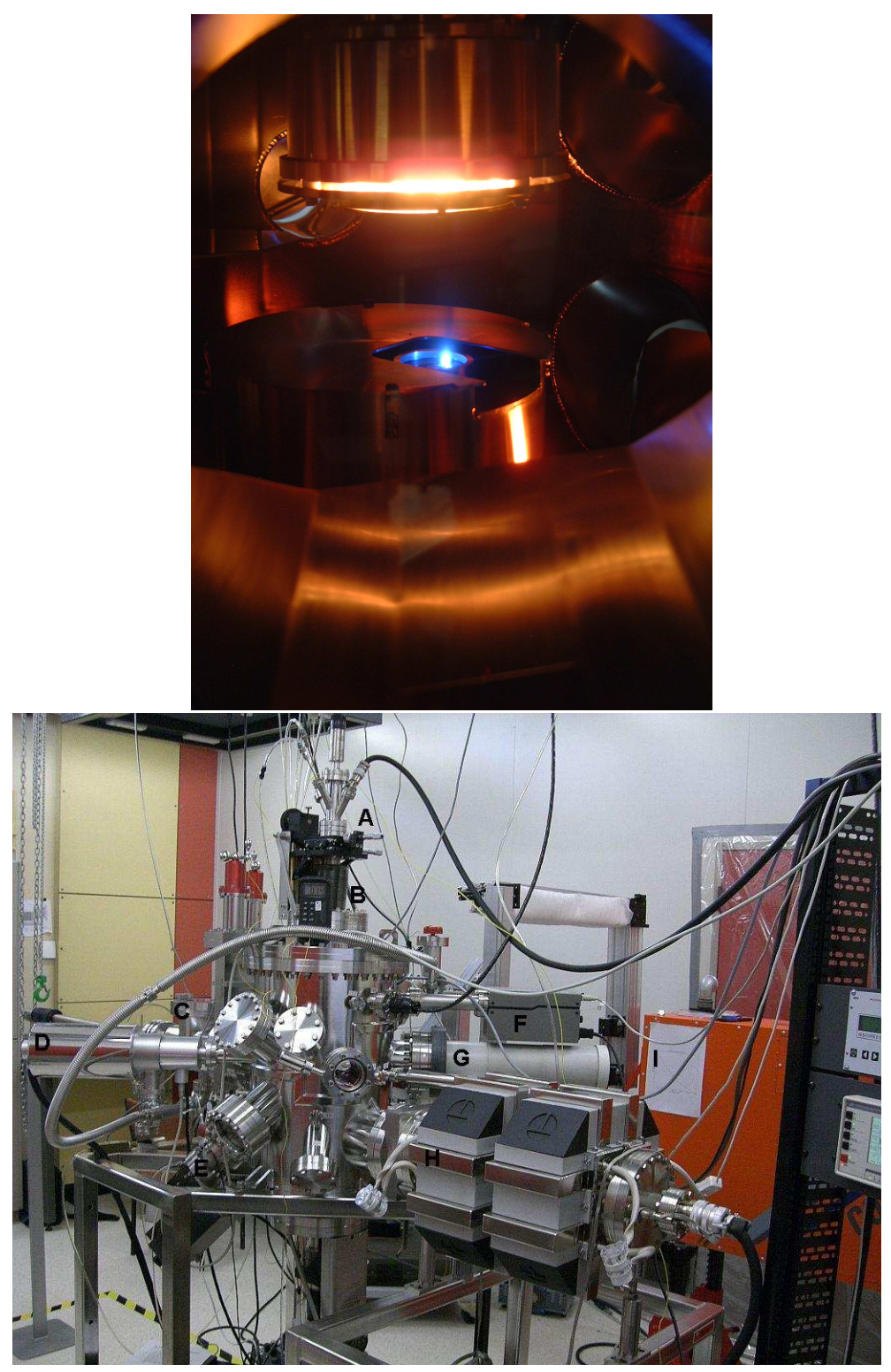

Figure 4.1: Top: The inside of the chamber through a viewport. A target can be seen in the centre with a plume of ablated material coming off it towards the substrate, fixed above the target. Bottom: Outside of the chamber. 
tal quality of the samples as determined by RHEED. Because the RHEED is in situ, it is easiest to correlate growth parameters with crystal quality. It is perhaps more interesting to look at how sample growth impacts on the magnetic or electronic properties and this will be discussed where possible in the sections of this thesis dealing with the magnetism and transport properties respectively.

In the sample preparation, there were certain procedures that were common to all growths. Substrates were $10 \times 10 \times 0.5 \mathrm{~mm}$ yttria stabilised zirconia $\left(\left[\mathrm{Zr}, \mathrm{Y} \mathrm{O}_{2}\right.\right.$ with Zr:Y = 91:9), double side polished, purchased from MTI Corporation ${ }^{2}$. YSZ was chosen as a suitable substrate because it takes a face centred cubic structure, similar to the RENs, with a lattice parameter of $5.125 \AA$, just a few percent larger than GdN. Substrates were $99.99 \%$ pure, and packed in a 1000 grade clean room into 100 grade clean plastic bags. Substrates were subject to a standard ultrasonic degreasing procedure involving a five minute immersion in each of trichloroethylene, acetone and methanol, and then mounted on a molybdenum sample holder using an indium-tin liquid alloy. Once mounted in the growth chamber, the substrate and sample holder were subject to an outgassing at $800{ }^{\circ} \mathrm{C}$ until the water vapour in the chamber, monitored with a mass spectrometer, reached a minimum. This was typically about 60 minutes. This ensured any water vapour or other surface contaminants were removed from the surface before growth.

GdN and SmN had not been grown by PLD before, and so it was very important to investigate the space of growth parameters. Ideally, this would lead to the optimisation of film growth in terms of extended defects such as dislocations and grain boundaries, and point defects such as oxygen impurities and nitrogen vacancies. The following tables lay out the important parameters for all the growths. Samples were primarily capped with YSZ, which was ablated from a large single crystal in vacuum. A number of $S m N$ growths were capped with Aluminium nitride (AlN) ablated from a ceramic target in vacuum but this proved to be no more successful than YSZ at protecting the films in atmosphere.

\footnotetext{
${ }^{2}$ www.mtixtl.com
} 


\begin{tabular}{|c|c|c|c|c|c|c|}
\hline $\begin{array}{l}\text { Sample } \\
\text { ID }\end{array}$ & Substrate & $\begin{array}{l}\text { Growth } \\
\text { time: } \\
\text { GdN/cap } \\
\text { (min) }\end{array}$ & $\begin{array}{l}\text { Fluence } \\
\left(\mathrm{J} / \mathrm{cm}^{2}\right)\end{array}$ & $\begin{array}{l}\mathrm{N}_{2} \\
\text { pres- } \\
\text { sure } \\
\text { (mbar) }\end{array}$ & $\begin{array}{l}\text { Plasma } \\
\text { power } \\
(\mathrm{W})\end{array}$ & $\mathrm{T}_{\text {subs }}\left({ }^{\circ} \mathrm{C}\right)$ \\
\hline GdN01 & YSZ (100) & $30 / 15$ & 5 & $1.2 \mathrm{E}^{-4}$ & 200 & 700 \\
\hline GdN02 & YSZ (100) & $30 / 15$ & 5 & $1.0 \mathrm{E}^{-4}$ & 200 & 700 \\
\hline GdN03 & YSZ (100) & $30 / 15$ & 5 & $1.0 \mathrm{E}^{-4}$ & 200 & 700 \\
\hline GdN04 & YSZ (100) & $30 / 15$ & 5 & $1.0 \mathrm{E}^{-4}$ & 200 & 700 \\
\hline GdN05 & YSZ (100) & $30 / 15$ & 5 & $1.0 \mathrm{E}^{-4}$ & 200 & 800 \\
\hline GdN06 & YSZ (100) & $30 / 15$ & 5 & $1.0 \mathrm{E}^{-4}$ & 200 & 850 \\
\hline GdN07 & YSZ (100) & $60 / 15$ & 5 & $1.0 \mathrm{E}^{-4}$ & 200 & 850 \\
\hline GdN08 & YSZ (100) & $60 / 8$ & 5 & $5.0 \mathrm{E}^{-5}$ & 200 & 800 \\
\hline GdN09 & YSZ (111) & $60 / 8$ & 5 & $5.0 \mathrm{E}^{-5}$ & 200 & 800 \\
\hline GdN10 & YSZ (100) & $30 / 15$ & 5 & $2.5 \mathrm{E}^{-5}$ & 200 & 800 \\
\hline GdN11 & YSZ (111) & $30 / 15$ & 5 & $2.5 \mathrm{E}^{-5}$ & 200 & 800 \\
\hline GdN12 & YSZ (100) & $30 / 15$ & 5 & $2.5 \mathrm{E}^{-5}$ & 200 & 800 \\
\hline GdN13 & YSZ (111) & $30 / 15$ & 5 & $2.5 \mathrm{E}^{-5}$ & 200 & 800 \\
\hline GdN14 & YSZ (100) & $60 / 15$ & 7.5 & $1.0 \mathrm{E}^{-4}$ & 200 & 800 \\
\hline GdN15 & YSZ (100) & $60 / 15$ & 7.5 & $1.0 \mathrm{E}^{-4}$ & 400 & 800 \\
\hline GdN16 & YSZ (100) & $50 / 30$ & 7.5 & $1.0 \mathrm{E}^{-4}$ & 120 & 800 \\
\hline GdN17 & YSZ (100) & $120 / 30$ & 3 & $1.0 \mathrm{E}^{-4}$ & 200 & 750 \\
\hline GdN18 & YSZ (100) & $130 / 30$ & 2 & $1.0 \mathrm{E}^{-4}$ & 200 & $200 / 750^{2}$ \\
\hline GdN19 & YSZ (111) & $130 / 30$ & 2 & $1.0 \mathrm{E}^{-4}$ & 200 & $200 / 750^{2}$ \\
\hline GdN20 & YSZ (100) & $30 / 15$ & 5 & $1.0 \mathrm{E}^{-4}$ & 0 & 700 \\
\hline GdN21 & YSZ (100) & $30 / 15$ & 5 & $1.0 \mathrm{E}^{-4}$ & 0 & 20 \\
\hline GdN22 & YSZ (100) & $30 / 15$ & 5 & $1.0 \mathrm{E}^{-4}$ & 200 & 700 \\
\hline GdN23 & YSZ (100) & $30 / 15$ & 5 & $1.0 \mathrm{E}^{-4}$ & 200 & 20 \\
\hline GdN24 & YSZ (100) & $30 / 15$ & $5^{1}$ & $1.0 \mathrm{E}^{-4}$ & 200 & 700 \\
\hline GdN25 & YSZ (111) & $30 / 15$ & $5^{1}$ & $1.0 \mathrm{E}^{-4}$ & 200 & 700 \\
\hline GdN26 & YSZ (100) & $30 / 15$ & $5^{1}$ & $5.0 \mathrm{E}^{-5}$ & 200 & 700 \\
\hline
\end{tabular}

${ }^{1}$ Spot area and energy increased by factor of 3

${ }^{2}$ Low temperature GdN buffer layer grown for 10 minutes, followed by high temperature growth 


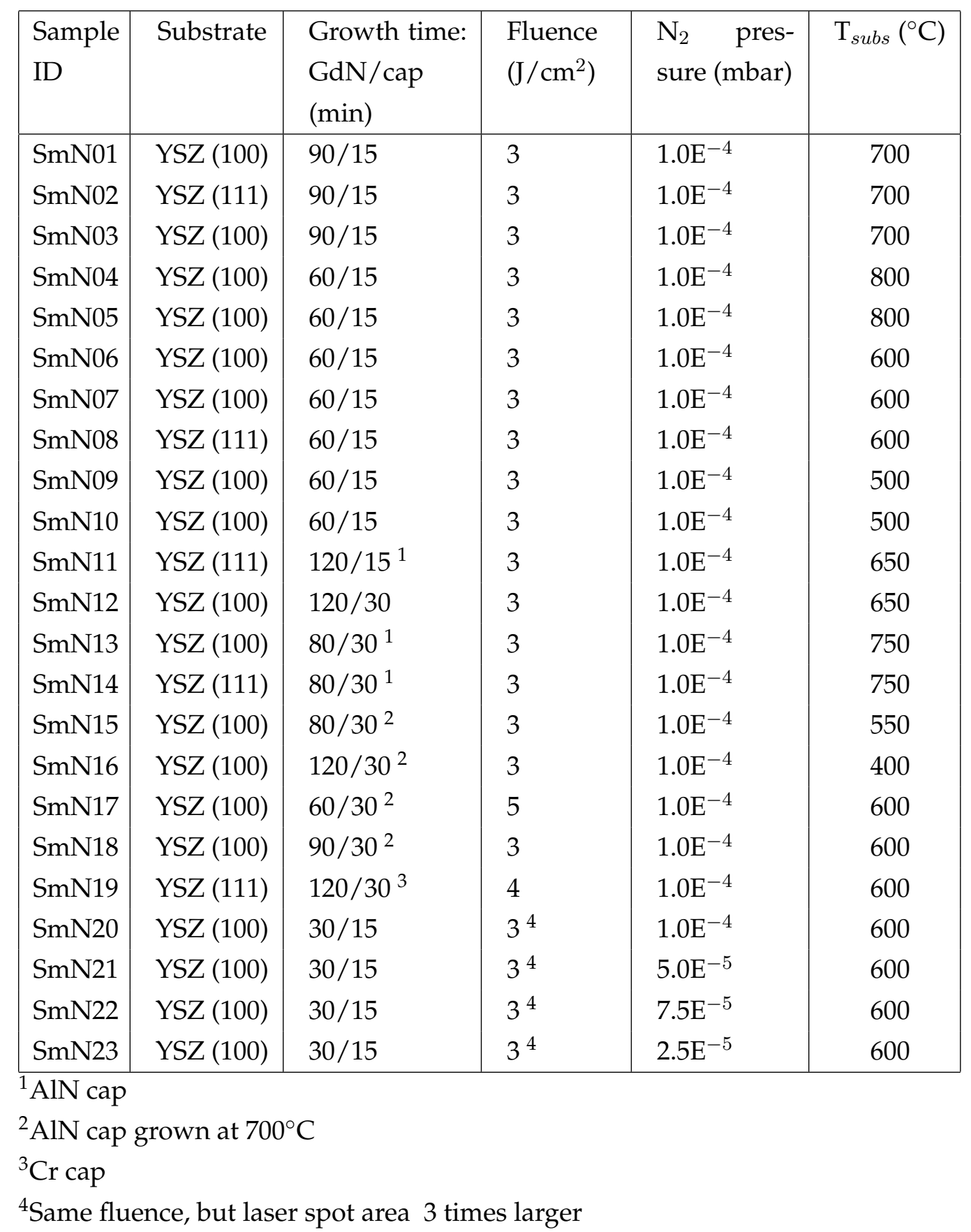




\subsection{Structural and surface characterisation}

In order to assess and compare the crystal quality of the films produced by PLD a number of characterisation techniques were used, both in situ and ex situ.

\subsubsection{Growth dynamics}

Reflection high energy electron diffraction measured in situ during growth gives useful information about the structure of the surface of the film. Figure 4.2 shows the diffraction patterns obtained at various times during growth of GdN [(a)-(c)] and $\mathrm{SmN}[(\mathrm{d})-(\mathrm{f})]$. The bright, narrow lines of $\mathrm{GdN}$ and $\mathrm{SmN}$ after 30 minutes of growth [(c) and (f)] are indicative of a well ordered surface. The bright spots visible within the lines imply the surface is rough as opposed to layer by layer growth. The width of the lines is inversely proportional to the coherence length, while the spacing between the lines is inversely proportional to the in-plane surface lattice parameter.

The full width at half maximum (FWHM) of the central streak can be analysed quantitatively as the growth progresses, and the final value is useful for comparisons of crystal quality between samples. Figure 4.3(a) shows the FWHM as a function of growth time for the two GdN samples with the lowest final FWHM. From the initial narrow line corresponding to the substrate there is an increase to a turning point after $1-2$ minutes of growth. The FWHM then decreases as the growth progresses until it reaches a minima, typically before 10 minutes of growth, and does not change for the remainder of the growth. The form of the behaviour is typical for all samples, GdN and $\mathrm{SmN}$, but the maxima and the subsequent narrowing varied from sample to sample.

The surface lattice parameter was also monitored during growth. The early stages of growth for typical GdN and $\mathrm{SmN}$ films are shown in Figure 4.3(b). From the initial substrate lattice constant of $5.12 \AA$ there is a sharp increase to roughly $5.4 \AA$ before the lattice begins to contract towards the bulk value of GdN. This increase consistently peaks at film thicknesses of $2-4 \mathrm{~nm}$ suggesting it is an interfacial phenomenon. Given the proven affinity of gadolinium for oxygen[18] and the mobility of oxygen in YSZ [47], it is likely that gadolinium oxide $\left(\mathrm{Gd}_{2} \mathrm{O}_{3}\right)$ is forming at the interface by taking oxygen from the substrate. The lattice con- 


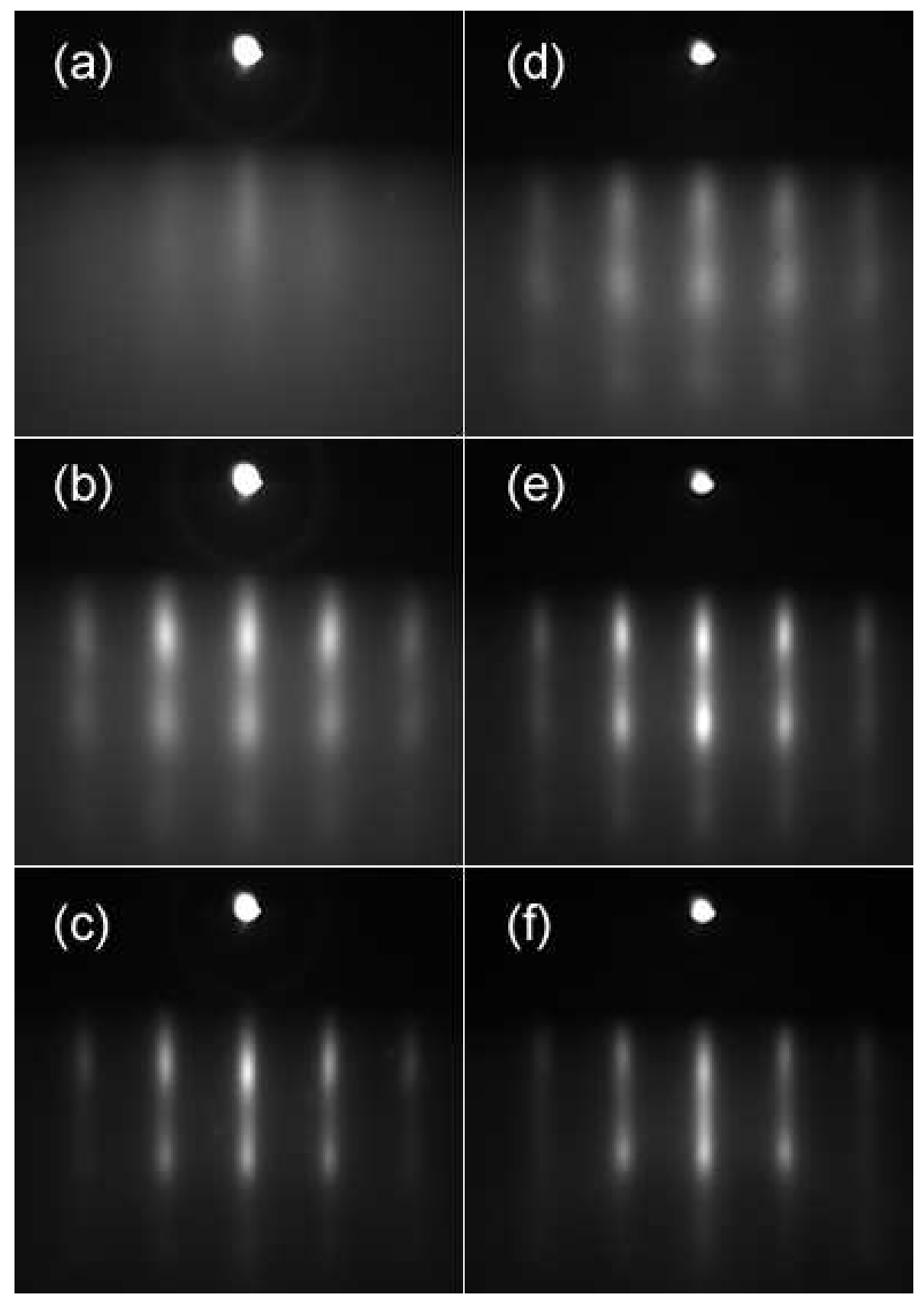

Figure 4.2: Rheed images for $\operatorname{GdN}[(\mathrm{a})-(\mathrm{c})]$ and $\mathrm{SmN}$ [(d)-(f)]. The rows correspond to images taken at 1 minute, 5 minutes, and 30 minutes. 

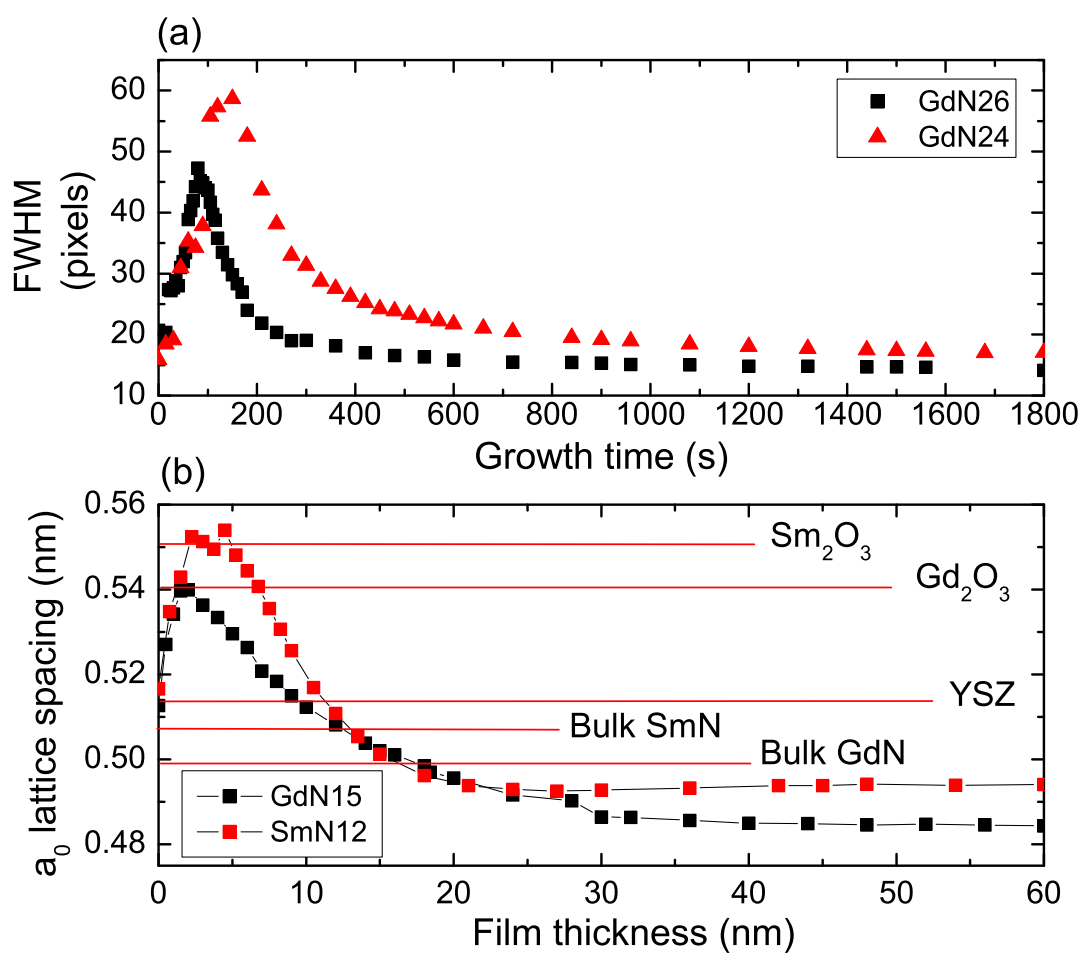

Figure 4.3: (a) The FWHM of the central RHEED streak plotted as a function of time for two GdN samples. (b) Lattice spacing as a function of film thickness for typical GdN and SmN growths. Expected lattice parameters are indicated with horizontal lines.

stant of approximately $5.4 \AA$ agrees with this conclusion: $\mathrm{Gd}_{2} \mathrm{O}_{3}$ in its cubic form takes the 'bixbyite' crystal structure with a lattice constant of $10.813 \AA$ [48]. The unit cell of this structure consists of $\mathrm{Gd}$ ions in a fcc lattice with a lattice parameter of $5.41 \AA$, with oxygen ions filling up the gaps in a manner that repeats every $10.813 \AA$, or every two fcc $\mathrm{Gd}$ cells along the $\langle 100\rangle$ direction. The early stages of $\mathrm{SmN}$ growth add further support to the oxide formation conclusion. A similar peak in lattice spacing was observed for all $\mathrm{SmN}$ films, but peaking at closer to $5.5 \AA ; \mathrm{Sm}_{2} \mathrm{O}_{3}$ has a lattice constant of $10.932 \AA$.

The final in-plane lattice parameter is always found to be smaller than the ex- 
pected bulk REN lattice parameter by $2-3 \%$. This is explained as a surface effect given the $\mathrm{XRD}$ results discussed below. However, the reasons for this behaviour are not yet well understood.

In order to further understand the early growth dynamics, a gadolinium film was grown in the absence of nitrogen. The relaxation of this film shows the same early growth behaviour as reported above for $\mathrm{GdN}$ and $\mathrm{SmN}$. This tells us that the early growth behaviour is not controlled by the presence of nitrogen, but rather the interaction of the rare earth ion with the substrate. This experiment supports the claim that $\mathrm{Gd} 2 \mathrm{O} 3$ is formed near the substrate due to the mobility of oxygen in the YSZ. As the film thickness increases, the availability of oxygen at the growth surface decreases. For films grown in a nitrogen atmosphere, the nitride then begins to form and the lattice spacing changes to reflect this. In the case of the Gd growth, it is interesting to note that the lattice structure we observe at the end of the growth is epitaxial fcc with a lattice parameter of roughly $5.08 \AA$. Gd metal usually takes a hexagonal close packed structure [49].

\subsubsection{XRD - GdN}

XRD measurements on GdN films confirm the epitaxy. Figure 4.4 shows that only the $[00 n]$ lines are observed as expected for cubic epitaxial films grown on (001) oriented substrates. The GdN [002] and [004] peaks are labelled with an arrow, while the corresponding substrate peaks are marked with a grey box. The inset shows the region around the [002] peak in more detail. The origin of the broad peak around $50^{\circ}$ is unknown, but possible explanations include the capping layer, or the film-substrate interface. The lattice parameter has been found to be $5.00 \pm$ $0.02 \mathrm{~nm}$, in good agreement with the expected bulk value of $4.99 \mathrm{~nm}$ [5]. It is stressed that this is the spacing between planes, along the $c$ axis. From this we may argue that the anomalously small lattice parameter measured in plane by RHEED is in fact a surface effect, and is not representative of the bulk of the sample.

Performing a Scherrer analysis on the GdN peaks shows that these samples have a much larger coherence length than samples fabricated by most other methods (with the possible exception of epitaxial MBE grown films) [17, 28, 18]. GdN 


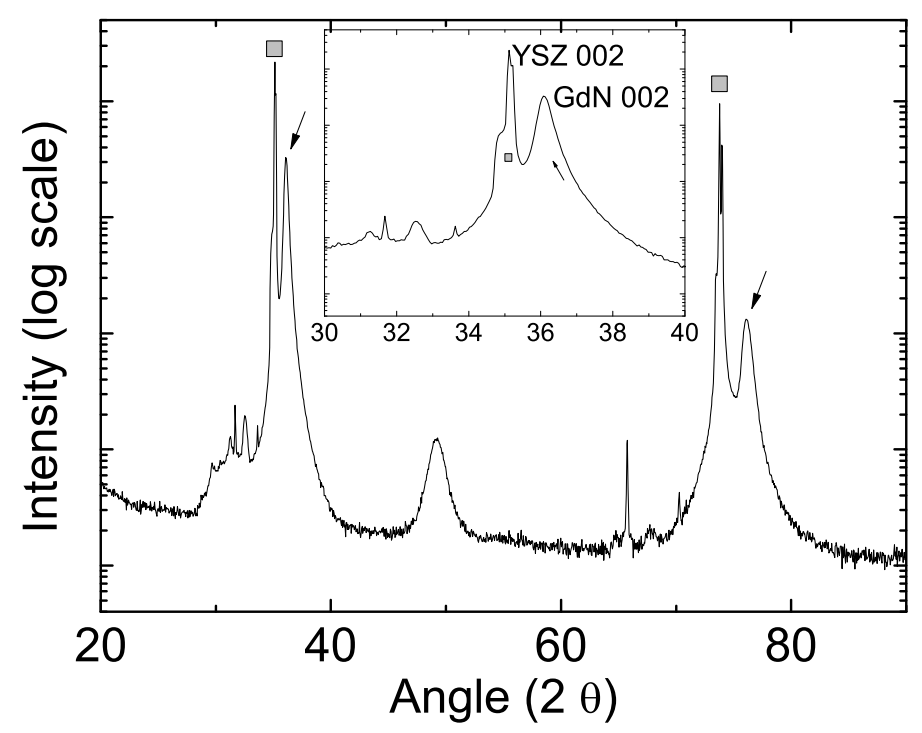

Figure 4.4: $2 \theta$ XRD scan on a typical GdN film. Substrate [00n] peaks are marked with a grey square, film [00n] peaks marked with arrows. Inset shows zoom on [002] peaks.

peaks were fitted with a Lorentzian lineshape and the FWHM value extracted. The quality of the fits was generally very good, leading to a small uncertainty. The coherence length along the $c$ axis ranges from $15 \mathrm{~nm}$ up to almost $30 \mathrm{~nm}$ in the best samples. There does not appear to be any clear correlation between coherence length and sample preparation. It does, however, correlate with the width of the RHEED pattern; a high surface coherence length implies a high bulk coherence length.

Rocking curves were obtained from selected samples to look at the uniformity of the $c$ axis distribution. Figure 4.5 shows rocking curves taken at $90^{\circ}$ intervals about the film normal. The broad peak corresponds to $\mathrm{GdN}$, while the sharp peak corresponds to the tail of the substrate peak. From the FWHM of the Gdn peak, there is a significant mosaic spread of $2.8 \pm 0.2^{\circ}$. This means that the $c$ axes of the crystallites are not all exactly colinear, but rather, that they are distributed about this direction. Mosaic spread is typically associated with a high dislocation 


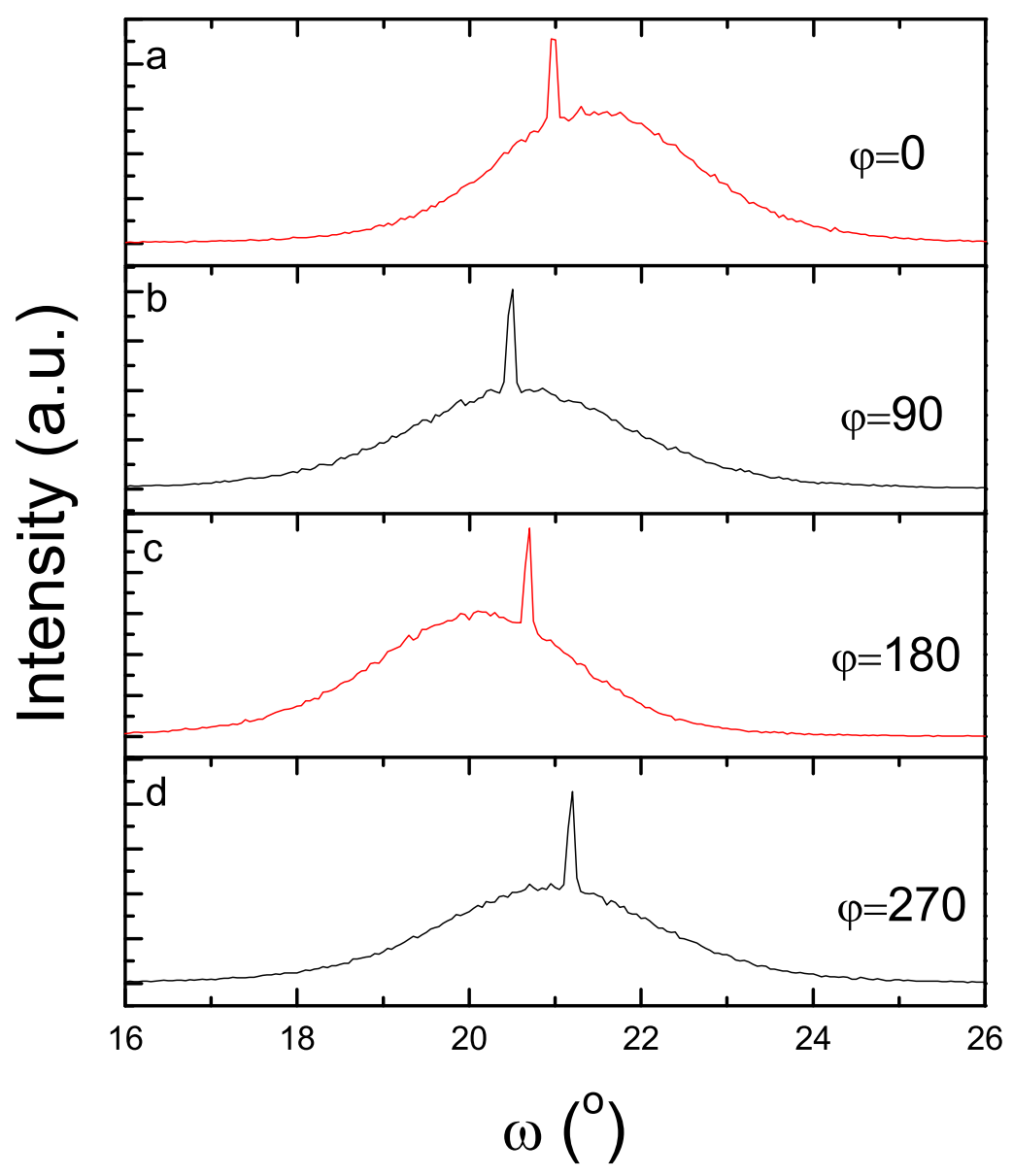

Figure 4.5: Rocking curves taken at various values of $\phi$ for a GdN film. 
density, which would explain the observations in these samples: with a lattice mismatch of the order of $2.5 \%$ we expect a large density of dislocations in the early stages of growth. The formation of an oxide buffer layer with an even larger lattice mismatch complicates things, but it is reasonable to expect that this would introduce further dislocations.

A second piece of information to come out of the rocking curves is that there is a fixed tilt of the film c-axis with respect to the substrate lattice. There is often some degree of tilt between the substrate lattice and the actual substrate plane due to polishing and preparation conditions. The substrate manufacturers quote the lattice orientation to within $0.5^{\circ}$ and we observed it to be roughly $0.4^{\circ}$. Taking this into account, we found that there is $0.74^{\circ} \pm 0.10^{\circ}$ between the substrate lattice normal, and the film lattice normal. The projection of this tilt into the plane lies at an angle of about $15^{\circ}$ from the [100] direction, and at about $50^{\circ}$ from the projection of the substrate tilt (relative to the actual substrate surface). This is shown graphically in Fig. 4.5 where the centre of the broad peak corresponding to GdN shifts as the sample is rotated about the normal.

\subsubsection{XRD - SmN}

XRD measurements on $\mathrm{SmN}$ are less simple to interpret. Due to the reduced lattice mismatch of about $1 \%$ the $\mathrm{SmN}[00 n]$ peaks are obscured by the strong substrate peak, shown in Fig. 4.6(a). A small [111] peak is visible even in the epitaxial film indicating a small amount of orientational disorder. The SmN [002] peak can be seen by using the fact that the film is tilted with respect to the substrate and rocking the sample to an angle that optimises the SmN peak with respect to the substrate peak. In Fig. 4.6(b) the $\mathrm{SmN}$ peak is clear, with a lattice constant of $5.08 \pm 0.02 \mathrm{~nm}$. The FWHM of the rocking curve shown in Fig. 4.6(c) indicates the mosaic spread is of a similar order of magnitude to that of $\mathrm{GdN}$. 

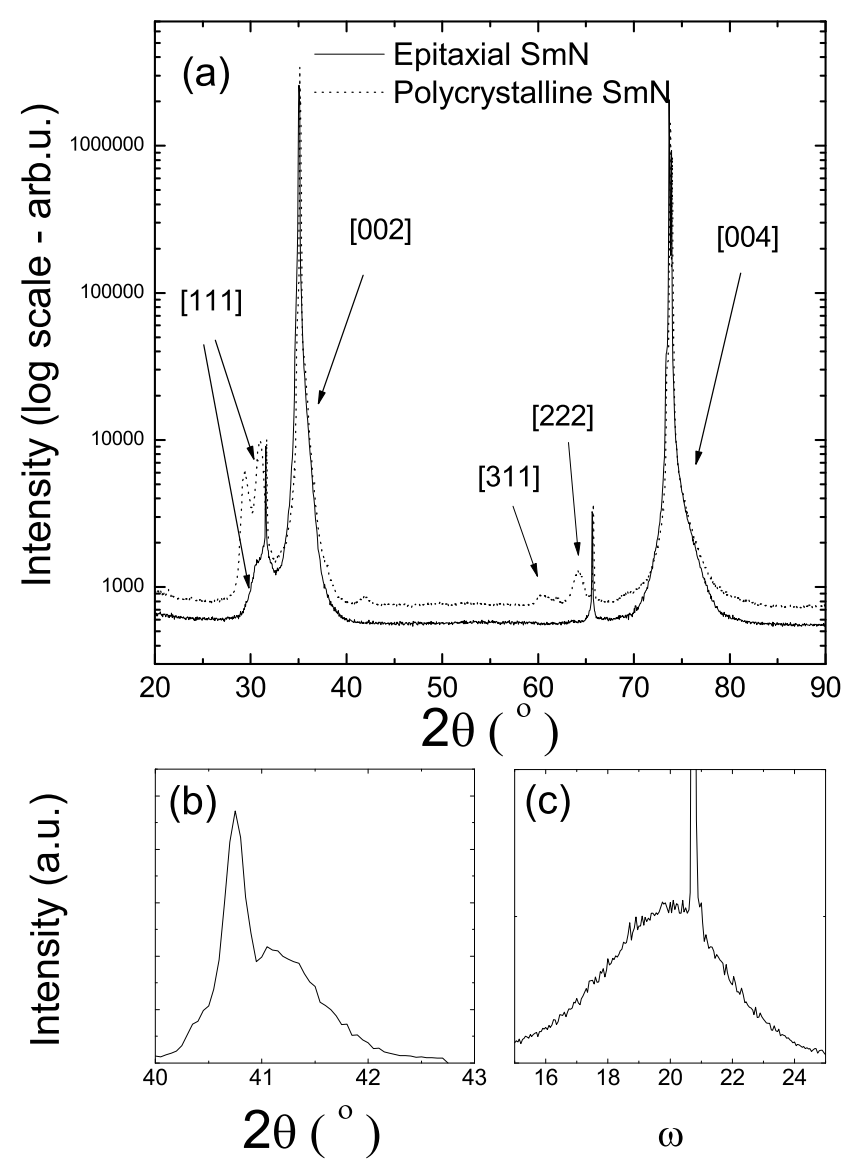

Figure 4.6: (a) $2 \theta$ XRD for two PLD SmN films taken using Co radiation. The dotted line corresponds to a film grown at high temperature, found to be polycrystalline, although still textured. (b) A closer look near the [002] line shows that the SmN peak (broad) can be separated from the substrate (sharp). (c) shows a rocking curve indicating significant mosaic spread. 


\subsubsection{Imaging}

Metallic droplets are often a problem in films grown by PLD and a number of causes have been identified in the literature [50]. The two most likely causes in the growths reported here are splashing and target surface roughness [44]. The first occurs due to the boiling of small molten regions of the target surface following the shock heating from the laser [45]. The second is related to a rough surface, facilitating the ejection of target material [44, 46].

In order to check for the presence of droplets in the films, images were taken using a high magnification optical microscope. Images are of GdN samples on YSZ substrates, where the indium on the back had been carefully removed with fine sandpaper; selected images are shown in Figure 4.7. We see evidence of droplets ranging from 0.1 to $3 \mu \mathrm{m}$ in diameter with a density on the order of $10000 / \mathrm{mm}^{2}$. From the five samples analysed for the density of droplets, two trends can be tentatively identified. The density of droplets appears to increase with both laser fluence, and growth time. Both of these are consistent with common causes of droplets outlined above, namely splashing, and surface roughness. A more detailed analysis is required to draw more concrete conclusions.

Images (a) and (b) show a GdN sample some months after growth and it is clear that some degradation has occurred. It appears that the micron sized inclusions (the small specs in image (b)) act as seeds for film degradation. Images (c) and (d) show another GdN film, but the images were taken soon after growth. While the droplets are clear, there is no ring of degraded film around them. As well as being fresher at the time of imaging, the sample in images (c) and (d) has a capping layer more that 3 times thicker than the sample shown in (a) and (b) which is expected to help prevent oxidisation. A SmN sample shown in images (e) and (f) is similar to the first GdN sample in that oxidisation has started to spread from the site of the micron sized inclusions.

Atomic force microscope images on a GdN sample confirm the presence and size distribution of the inclusions. Figures 4.8 (a)\&(c) show an area of roughly $18 \mu m^{2}$ with particulates ranging from 0.1 to $3 \mu m$ in diameter. A step can be seen in image (c) and up closer in image (d). The height of this step was measured to be $25 \mathrm{~nm}$ which suggests that it is in fact the capping layer, which has a thickness of this order. This looks very much like what was seen in the optical images in Fig. 
4.7. It appears that it is the capping layer that degrades around the particulates, and this would allow the REN to oxidise and degrade. Figure 4.8(b) shows a close-up view of a large droplet, measured to be $4 \mu \mathrm{m}$ wide and $0.8 \mu \mathrm{m}$ high. 


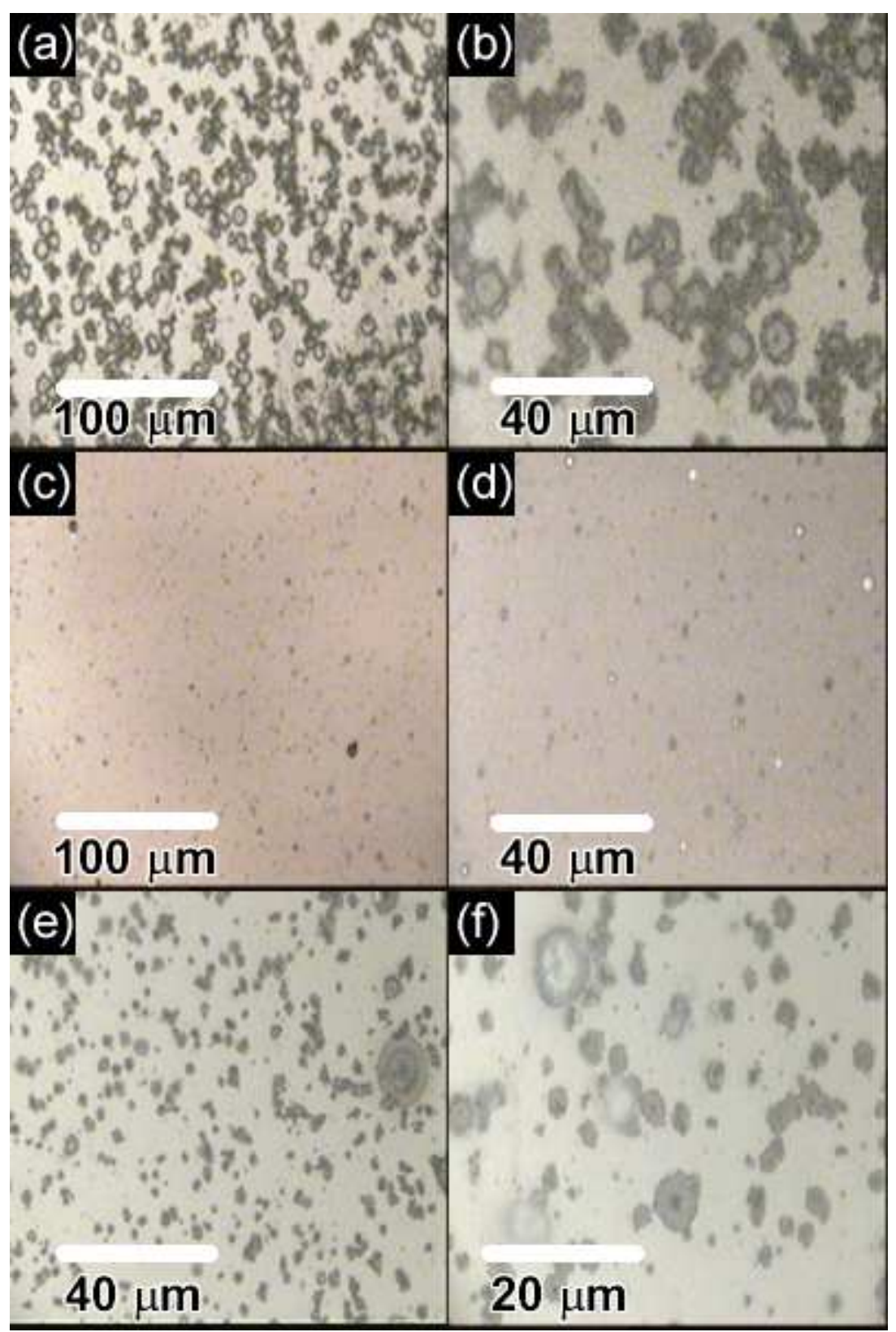

Figure 4.7: Optical microscope images of some PLD samples. (a,b) GdN08, months after growth. (c,d) GdN18, days after growth. (e,f) SmN, weeks after growth. 


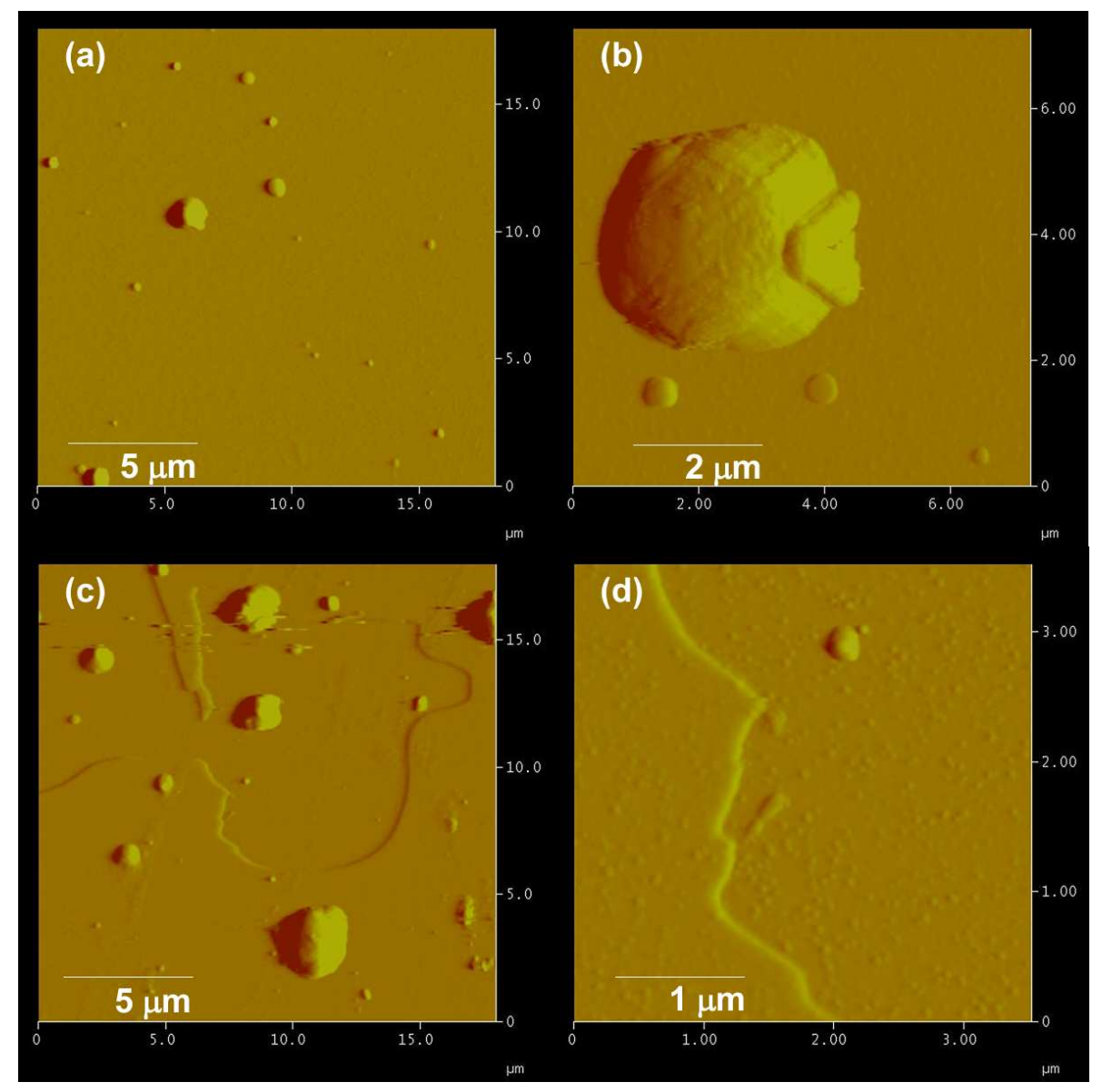

Figure 4.8: AFM images of a GdN sample surface. 


\subsection{Conclusions}

SmN and GdN films were grown at a range of temperatures from $20^{\circ} \mathrm{C}$ to $850{ }^{\circ} \mathrm{C}$ to establish the optimum growth temperature. The full width at half maximum (FWHM) of the central RHEED streak gives an indication of the coherence length, and hence the quality, of the surface layer. Comparison of the FWHM data for films grown with different parameters was used to optimize the film growth. For $\mathrm{SmN}$, crystal quality depends strongly on the growth temperature, as illustrated in Fig. 4.9 and from this, we conclude that $\mathrm{SmN}$ crystal quality is optimized for a growth temperature of $600 \pm 100^{\circ} \mathrm{C}$. Samarium has a vapour pressure of $1.3 \times 10^{-4} \mathrm{mbar}$ at $573^{\circ} \mathrm{C}[51]$, allowing us to grow at temperatures where excess $\mathrm{Sm}$ can be re-evaporated from the substrate. For $\mathrm{Gd}$, with a vapour pressure of $1.3 \times 10^{-4}$ mbar at $1175{ }^{\circ} \mathrm{C}$, we cannot heat the substrate to the temperature at which excess Gd may be re-evaporated. The FWHM vs. growth temperature for GdN shows no minima in the range of temperatures investigated, and this is probably because, based on what we observe for $\mathrm{SmN}$, the optimum growth temperature lies above that range.

The nitrogen flowing into the chamber during growth was activated with a plasma source operating at powers from 0 (off) to $400 \mathrm{~W}$ for some of the GdN growths. The majority of $\mathrm{GdN}$ and all $\mathrm{SmN}$ growths were done at $200 \mathrm{~W}$, in the middle of the range of available powers. High RF powers tend to increase the ratio of atomic to excited nitrogen, while low RF power decreases the total amount of excited nitrogen [52], both of which can be detrimental to film growth, as is observed below. GdN20 \& 21 were grown with no plasma, and were found to have FWHM a factor of two greater than those of the best samples grown. Samples grown with plasma powers of $120 \mathrm{~W}$ and $400 \mathrm{~W}$ (GdN15 \& GdN16) also had FWHM larger than that of an otherwise identical sample grown with $200 \mathrm{~W}$ (GdN14). From this series of growths, we can say that $200 \mathrm{~W}$ seems to be the optimum plasma power. It is also worth noting that two of the low power samples (16 $\& 20)$ were strained at the termination of growth: the lattice parameter measured by RHEED was still a few percent higher than the expected bulk value. Both of these samples delaminated on venting the chamber. The poor quality of the 'no plasma' growths relates back to the TED growths, which are all grown with no 


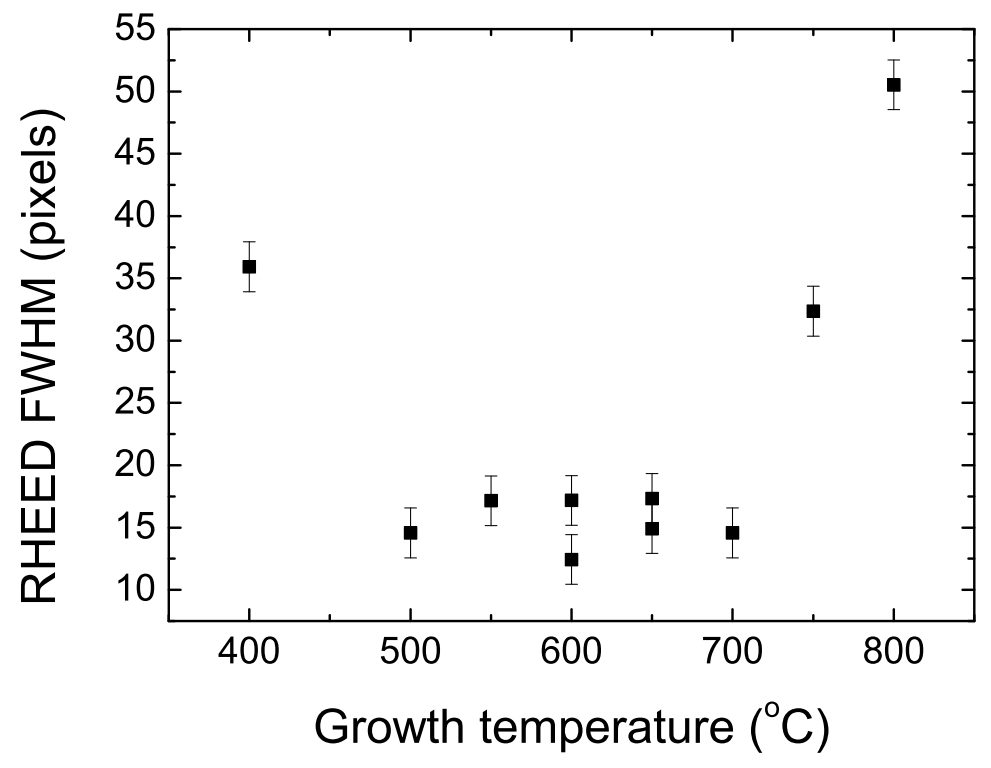

Figure 4.9: Optimisation of the growth temperature for $\mathrm{SmN}$ was determined by the FWHM of the central RHEED streak which is inversely proportional to the surface coherence length.

plasma. It would be interesting to see if TED samples can be improved by using plasma activated nitrogen.

The laser energy density at the target surface was varied for the growth of both materials: in GdN, from 2 to $7.5 \mathrm{~J} / \mathrm{cm}^{2}$ and in $\mathrm{SmN}$ from 3 to $5 \mathrm{~J} / \mathrm{cm}^{2}$. No relation was observed between the fluence and the FWHM in either material. A number of GdN growths were done with a fluence of $5 \mathrm{~J} / \mathrm{cm}^{2}$ but with the laser spot area made as large as possible. For these samples the total energy on the target was approximately three times larger than for samples grown with the standard spot area. Interestingly, these films had the lowest FWHM observed and evidence from FMR (Section 5.2) would suggest that these films contained less particulates and/or droplets as expected with the increased spot area.

The nitrogen pressure in the chamber was varied as shown in the growth tables. The principle aim of this was to observe changes in the transport or magnetic properties, which are not necessarily coupled to the crystal quality. Nonetheless, 
we can report that no significant, systematic variation in the RHEED FWHM can be attributed to the nitrogen pressure.

Films were grown on both (001) and (111) oriented YSZ substrates. The reflections seen by RHEED were consistent with the hexagonal pattern on the (111) face of a fcc crystal. The RHEED behaviour in the early stages of growth was similar for both substrates: the oxide lattice parameter was seen in both cases. The FWHM data indicates that for otherwise identical conditions, (001) substrates produce films of higher crystalline quality. 


\section{Chapter 5}

\section{GdN}

Having grown epitaxial GdN of high crystalline quality, we now look at the magnetic properties. Comparisons between samples prepared under different conditions, and even with different methods, could yield information on how the growth parameters relate to the magnetic properties, and therefore how to control the magnetic properties.

\subsection{Magnetometry in GdN}

SQUID magnetometry has been used as both a characterisation tool and to investigate the magnetic properties of GdN. SQUID measurements provide a direct and accurate measurement of the Curie temperature $\left(\mathrm{T}_{C}\right.$ - the temperature below which the material exhibits spontaneous magnetisation). Figure 5.1(a) shows zero field cooled (ZFC) measurements of PLD sample GdN02, and a GdN sample grown by simple thermal evaporation deposition (TED) for comparison. The abrupt upturn indicates the onset of ferromagnetic ordering at $70.5 \mathrm{~K}$, higher than any previously reported [53, 17, 28]. ZFC measurements on samples GdN24 and 26 in Fig. 5.1(b) show a very similar temperature dependence, with transition temperatures differing by just $1 \mathrm{~K}$ : GdN26 at $70 \mathrm{~K}$ and GdN24 at $71 \mathrm{~K}$. It is remarkable that all of the GdN samples grown by PLD have a transition temperature between 68 and $71 \mathrm{~K}$, despite widely varying growth parameters and crystalline 
quality. ${ }^{1}$

The Curie temperature is at the centre of the disagreement between theoretical and experimental results. Band structure calculations that describe the electronic structure so well consistently predict a low $\mathrm{T}_{C}$, ranging from 10 to $40 \mathrm{~K}[29,24]$. This does beg the question of whether the measured Curie temperature is not the intrinsic one, but rather due to nitrogen vacancies or carrier concentration, or some other contribution. However, it does appear that as sample preparation techniques have improved, there has been a tendency for the Curie temperature to be revised upwards. Early measurements by $\mathrm{Li}$ et al. suggested a $\mathrm{T}_{C}$ below 60K [53]. Similar results were reported by Luenberger et al.[17, 53] Subsequent measurements by Granville et al. increased it to $68 \mathrm{~K}$ [28]. The most recent measurements, reported here, and from Scarpulla et al. put $\mathrm{T}_{C}$ at $70 \mathrm{~K}[19,54]$. What is even more telling is that from the three latest reports, the carrier concentration varied by four orders of magnitude, while $\mathrm{T}_{C}$ varied by less than $5 \%$. This strongly suggests that the RKKY interaction is not significant, and that the value of $\mathrm{T}_{C}=70 \mathrm{~K}$ is approaching the intrinsic value for $\mathrm{GdN}$.

\footnotetext{
${ }^{1}$ Relative crystalline quality is determined by narrowness of RHEED lines and crystallite size from XRD.
} 

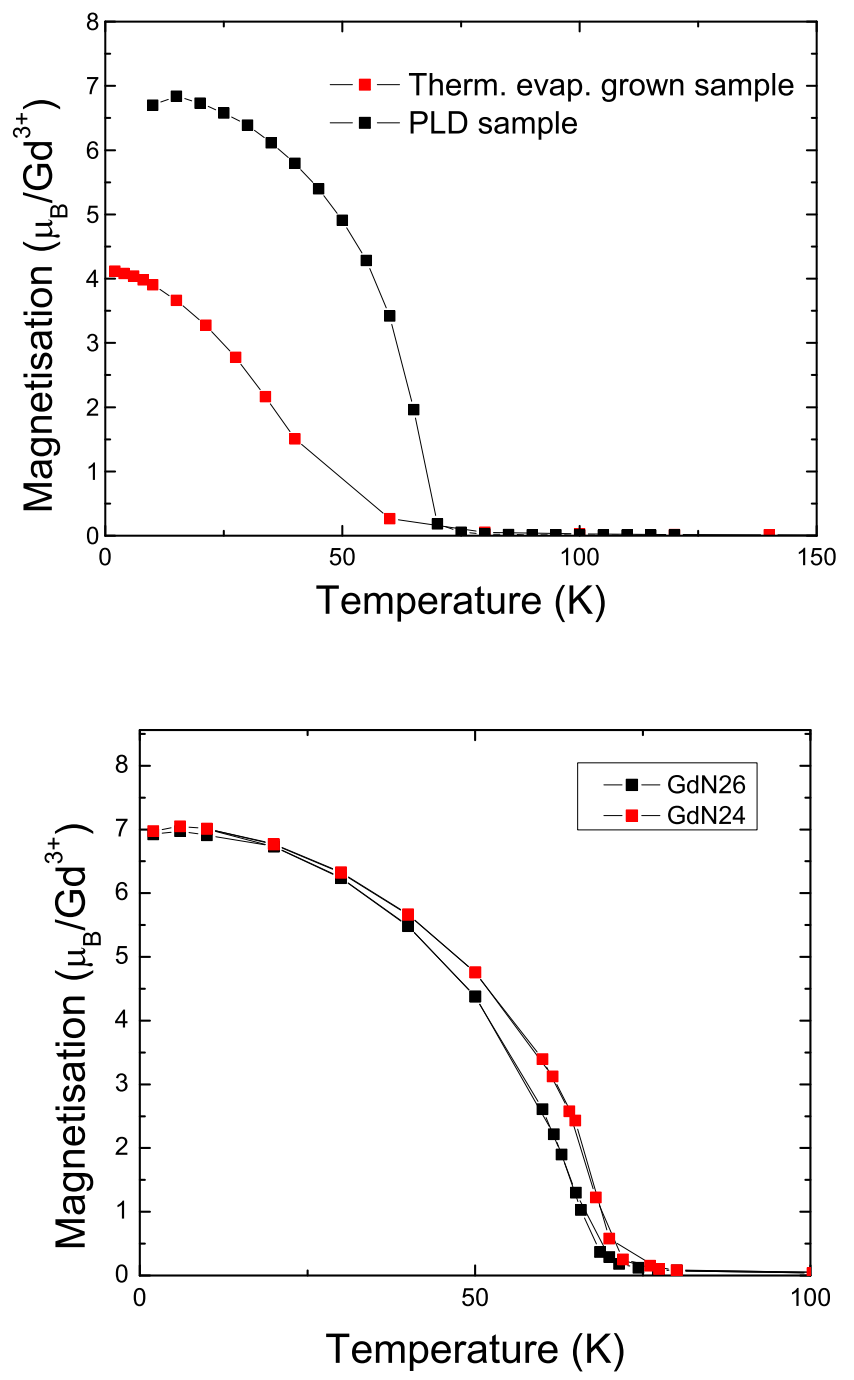

Figure 5.1: Figures showing the magnetisation with temperature. On the top there is a comparison between a PLD sample and a TED sample. The lower figure shows a comparison between two PLD sample prepared under different nitrogen pressures. 

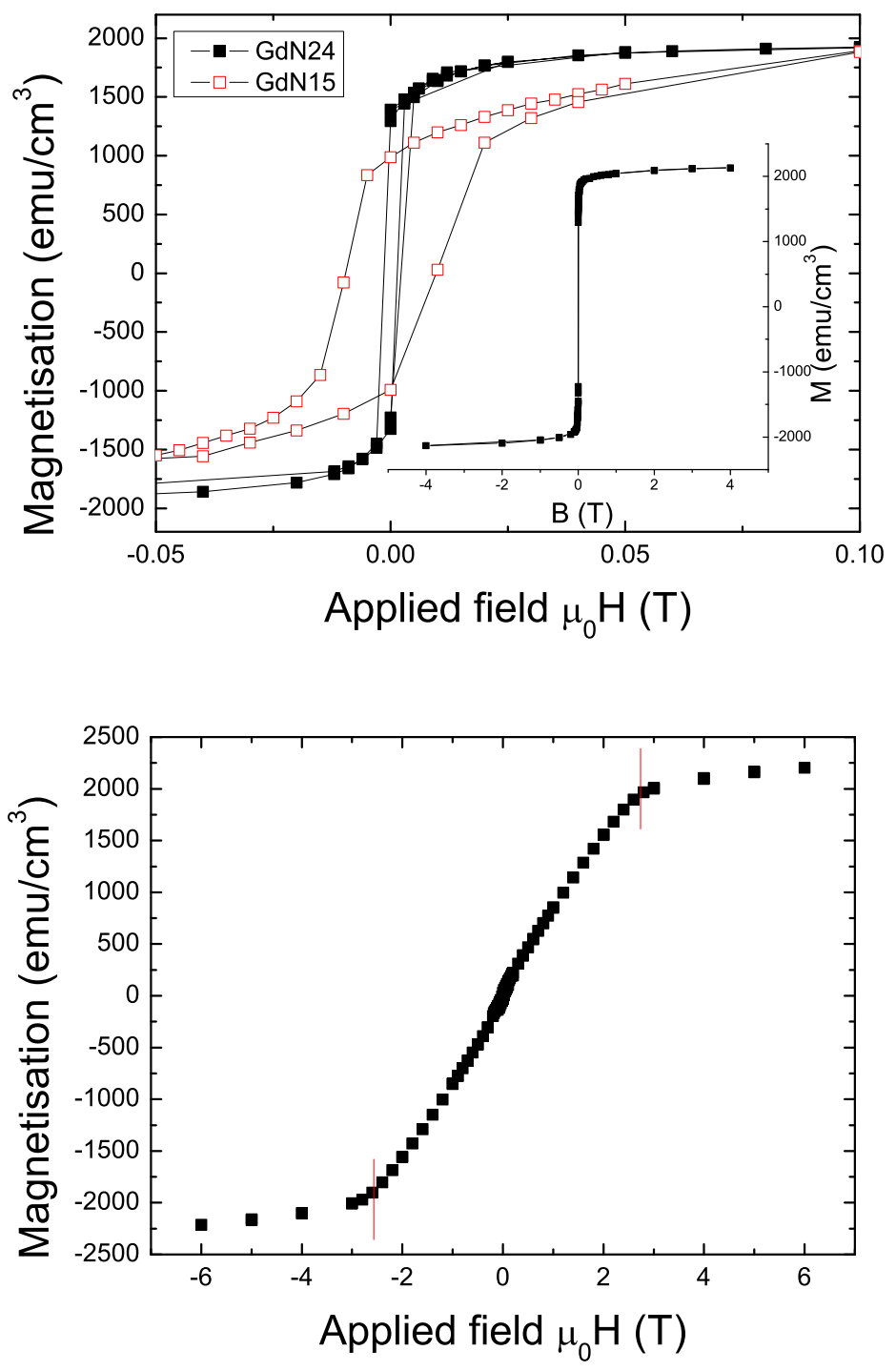

Figure 5.2: (a) Hysteresis loops at $10 \mathrm{~K}$ for GdN24 (narrow loop) and GdN15 (wide loop). Inset shows the full range for GdN24. (b) Magnetisation measured as a function of applied field, where the field is applied out of plane in order to observe the demagnetising field. 
Saturation magnetisation was found to be on the order of $7 \mu_{\mathrm{B}} / \mathrm{Gd}^{3+}$, or $2100 \mathrm{emu} / \mathrm{cm}^{3}$, as expected. For measurements with the applied field in the plane of the film at low temperature, the magnetisation saturates very quickly with applied field, as shown in Figure 5.2, and the response is isotropic in plane; the magnetic field can be applied along any in plane direction. In order to determine the magnetisation, the volume of the sample must be known (SQUID magnetometry measures the total moment of the sample at a given temperature and applied field). We cannot measure the thickness of the samples in situ, and estimates carry significant uncertainties. The magnetisation may, however, be determined from the demagnetising field, and this has the additional benefit of providing an estimate of the thickness. The demagnetising field is related to the thin film geometry, and is a field that appears perpendicular to the film plane, in response to an out of plane magnetisation of the sample. The demagnetising field (in Oersteds) is related to the magnetisation (in emu $/ \mathrm{cm}^{3}$ ) by $H_{D}=4 \pi M$. This can be measured by field loops with the field applied out of plane. The inflection points at positive and negative fields are determined as shown in Fig. 5.2(b) and averaged. The results are summarised in the table below.

\begin{tabular}{|c|c|c|c|c|}
\hline $\mathrm{GdN}$ & $H_{D}(\mathrm{Oe})$ & $\mathrm{M}\left(\mathrm{emu} / \mathrm{cm}^{3}\right)$ & $\mathrm{M}\left(\mu_{B} / \mathrm{Gd}^{3+}\right)$ & Thickness $(\mathrm{nm})$ \\
\hline 08 & 25700 & 2045 & $6.9 \pm 0.3$ & 80 \\
24 & 26600 & 2110 & $7.1 \pm 0.3$ & 55 \\
26 & 27000 & 2150 & $7.2 \pm 0.3$ & 60 \\
\hline
\end{tabular}

As mentioned above, in the PLD growth system, there is no way to monitor the thickness of the film in situ. Oscillations in the intensity of the RHEED pattern, which can often be used to count the atomic layers of a growth as they form, were not observed. Rutherford backscattering was used measure the thickness of a sample, and this can then be related to the growth conditions, from which we may get a rough estimate the thickness of other samples. GdN15, grown for 60 minutes with a fluence of $7.5 \mathrm{~J} / \mathrm{cm}^{2}$ was determined to be $120 \pm 5 \mathrm{~nm}$ thick, corresponding to a growth rate of $2 \mathrm{~nm} /$ minute. It is interesting to compare this measured thickness to the effective magnetic thickness. Knowing the area of the sample, the magnetisation, and the saturation moment, we can estimate the thickness of the magnetic layer that would give the measured moment. This is found 
to be $60 \pm 20 \mathrm{~nm}$, where the large uncertainty arises primarily from the measurement of the sample area.

The magnetic layer is significantly thinner than the total film thickness. From table 5.1, the calculated magnetic thickness of samples GdN24 and 26 is significantly smaller than expected based on the growth parameters. The trend continues for GdN08, GdN19, and GdN21. This implies there is a magnetically 'dead' layer up to about $40 \mathrm{~nm}$ thick in $\mathrm{GdN}$; some portion of the film that contributes no magnetic signal. This conclusion is presented with some caution as it is based on just one actual measurement of sample thickness. Although it is in fact what one would expect given the observations from RHEED relating to the formation of a non-magnetic oxide buffer layer at the substrate-film interface.

Hysteresis loops at low temperature in Figure 5.2(a) show a very narrow hysteresis and small coercive field. We have observed a correlation between the crystallite size ${ }^{2}$ and $H_{C}$. Larger crystallites correspond to a lower coercive field, which gives some clues as to the origin of the coercive field.

\footnotetext{
${ }^{2}$ We refer to the coherence length as 'crystallite size' for simplicity. The coherence length could in fact be an indication of the distance between extended dislocations rather than actual grain boundaries.
} 


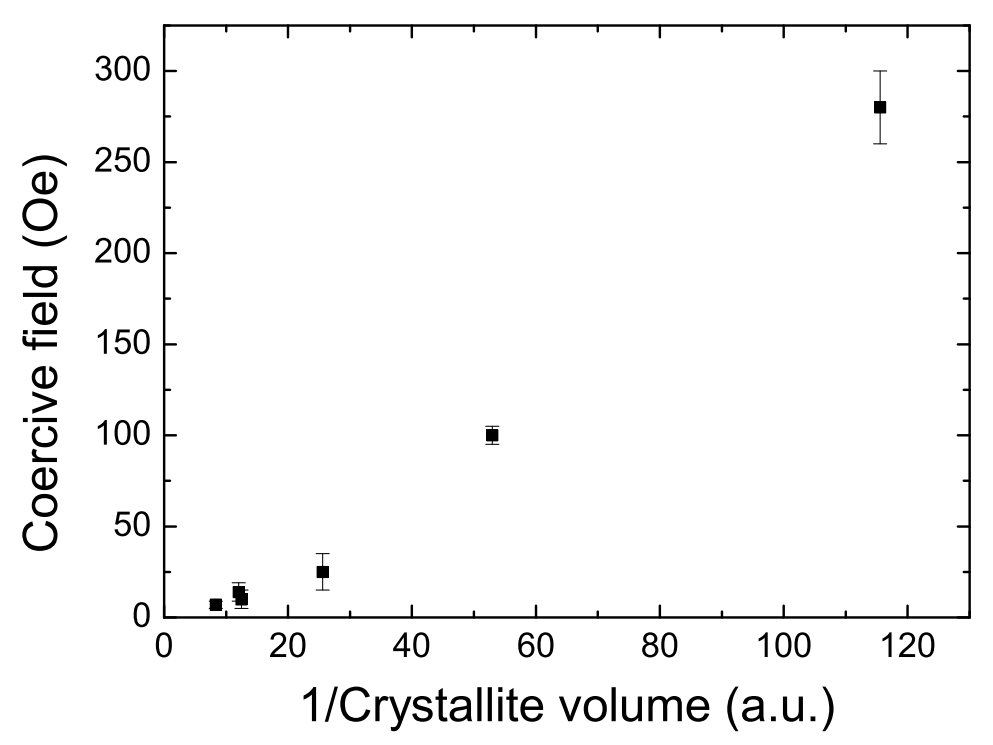

Figure 5.3: The measured coercive field against the inverse of crystallite volume for all samples for which both analyses were done. 
Crystallite diameter along the $c$ axis (out of plane) is determined by Scherrer analysis of XRD data (call this quantity $\zeta_{z}$ ). The sample volume could be estimated from this by assuming spherical crystallites, but there is no evidence to suggest the crystallites take this form. From the RHEED, measured during growth, the full width at half maximum (FWHM) is inversely proportional to the surface coherence length or crystallite size (call it $\zeta_{x}$ ). We know from the RHEED that this coherence length is isotropic on the surface, and so it would be reasonable to extract something proportional to crystallite volume as $\zeta_{x}^{2} \times \zeta_{z}$. The coercive field is plotted against the inverse of this quantity in Figure 5.3 and shows a near linear relationship. The error bars reflect the uncertainty in extracting the coercive field from the SQUID data.

There are a number of theories concerning the origin of the coercive field. Nucleation is one possible explanation: in addition to the anisotropy fields that must be overcome to nucleate a reversal of magnetisation (as per the StonerWohlfarth model), reversal can nucleate at localised magnetic inhomogeneities or defects. This is typically presented as a solution to Brown's paradox, explaining why the measured coercive field can be so much less than the anisotropy fields $[38,55]$. Under this interpretation, samples with high magnetic or structural disorder would show smaller coercive fields. This could also explain the relationship between coercive field and crystallite volume: it is statistically more likely that a larger crystallite will have a defect that nucleates a reversal of the magnetisation.

We may consider an alternative explanation by looking to the values of magnetocrystalline anisotropy determined by FMR (Section 5.2). For GdN24 we find the magnetocrystalline anisotropy field to be 16 Oe along the [111] direction, while the coercive field is measured to be $14 \mathrm{Oe}$. While the close agreement of these small values is probably chance, the fact that they are of the same order of magnitude indicates that, at least in this sample, there is no need to invoke nucleation to explain the coercive field; the coercive field has a lower limit approximately equal to the anisotropy. Larger coercive fields in other samples may be attributed to pinning of magnetic domain walls by defects, which would explain why samples with larger crystallite volume and sharper RHEED patterns exhibit smaller coercive fields. So we have here two opposing explanations of the coercivity in GdN: the first using the idea of nucleation where defects reduce $\mathrm{H}_{C}$, 
and the second using the anisotropy field and magnetic domain wall pinning, where defects increase $\mathrm{H}_{C}$. It is likely that both mechanisms are playing a role in determining the coercive field in GdN samples.

\subsection{Ferromagnetic resonance in $\mathrm{GdN}$}

The temperature dependence of the FMR signal contains information about the magnetisation of the sample: the intensity of the FMR signal is proportional to the total magnetisation of the sample [30], and the resonance field changes as a function of temperature. FMR spectra taken on GdN24 for a fixed, in-plane orientation at a range of temperatures are shown in Figure 5.4(a). As the temperature increases, the resonance shifts to the right, to higher fields. This is due to the decrease in the magnetisation of the sample, which leads to a change in the demagnetising field, $4 \pi M$, and hence a decrease in the effective field inside the sample. In Fig. 5.4(b), the normalised intensity of the FMR signal is plotted as a function of temperature. It is clear from the plot that the intensity follows approximately the magnetisation. As previously mentioned, the signal we measure is in fact the first derivative of the energy absorption curve. In order to calculate the integral of the signal, it was necessary to numerically integrate the measured signal twice. This was performed in OriginLab by first integrating the measured signal with no processing, and then using the 'peak analyzer' ${ }^{3}$ toolbox to subtract a baseline and integrate the resulting Lorentzian peak. This measurement confirms the $\mathrm{T}_{C}$ of approximately $70 \mathrm{~K}$ and hence the sample integrity, although it is not as precise as a SQUID measurement.

The angular dependence of the FMR signal at $4 \mathrm{~K}$ in GdN24 is plotted in Figure 5.5(a) (black squares). Recall this measurement is made by rotating the sample with respect to the applied magnetic field and extracting the resonance field as a function of angle out of the sample plane. The first feature to note is the very late and steep up turn of the resonance field as the applied field nears the out of plane orientation. This can be explained by the increase in the demagnetising field (proportional to the out of plane magnetisation) which acts to diminish the total internal field. The externally applied field must therefore be greater to attain

\footnotetext{
${ }^{3}$ Origin8: Analysis-Peaks and Baseline.
} 

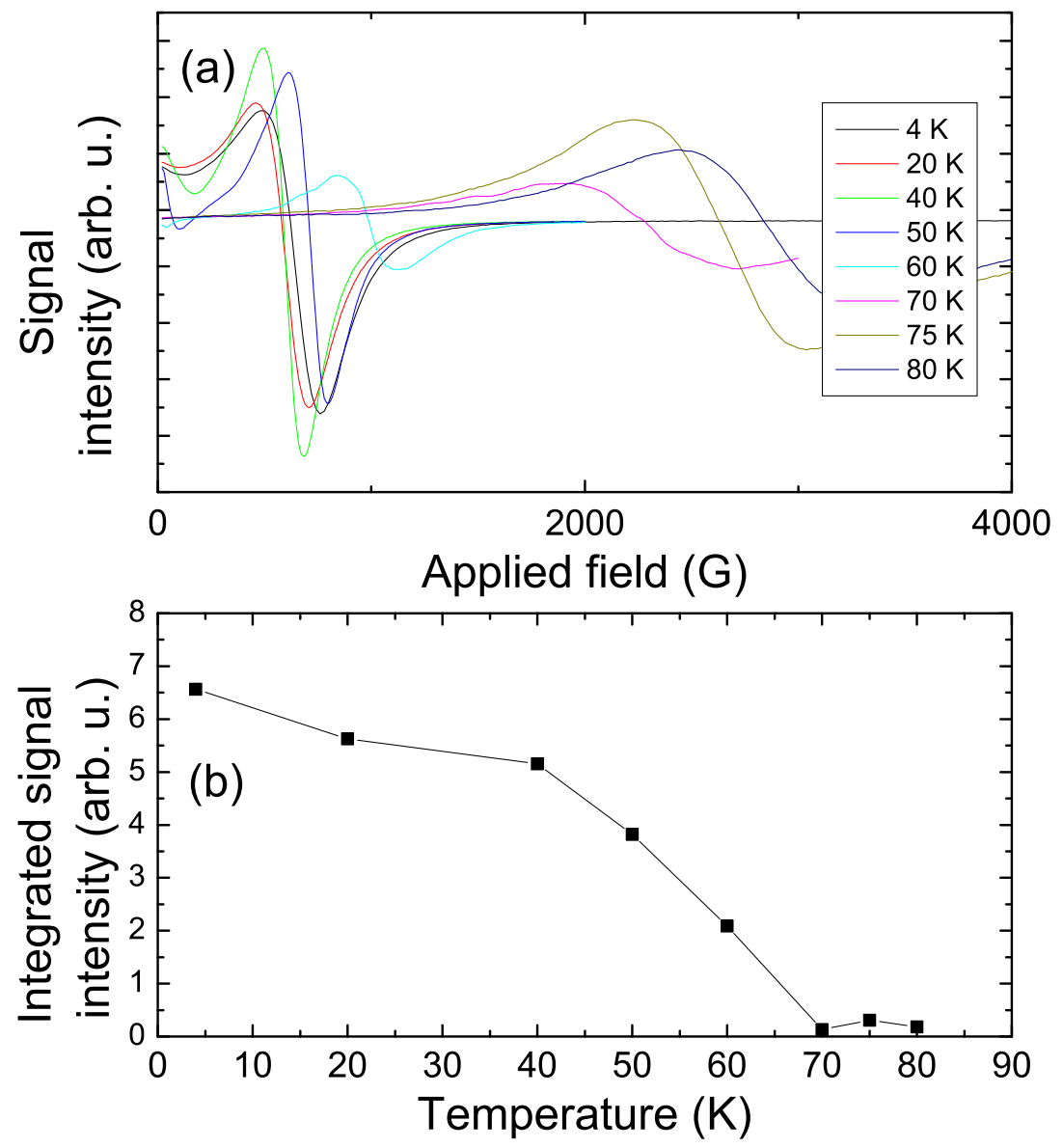

Figure 5.4: (a)GdN24 FMR spectra taken with the field applied in plane at a range of temperatures. (b)Double integral of the measured FMR signal versus temperature, which follows the magnetisation.

the Zeeman splitting required for resonance, and the resonance is then observed at higher applied fields. The conclusion to draw from this is that the plane of the film is the overwhelming easy plane of magnetisation. 


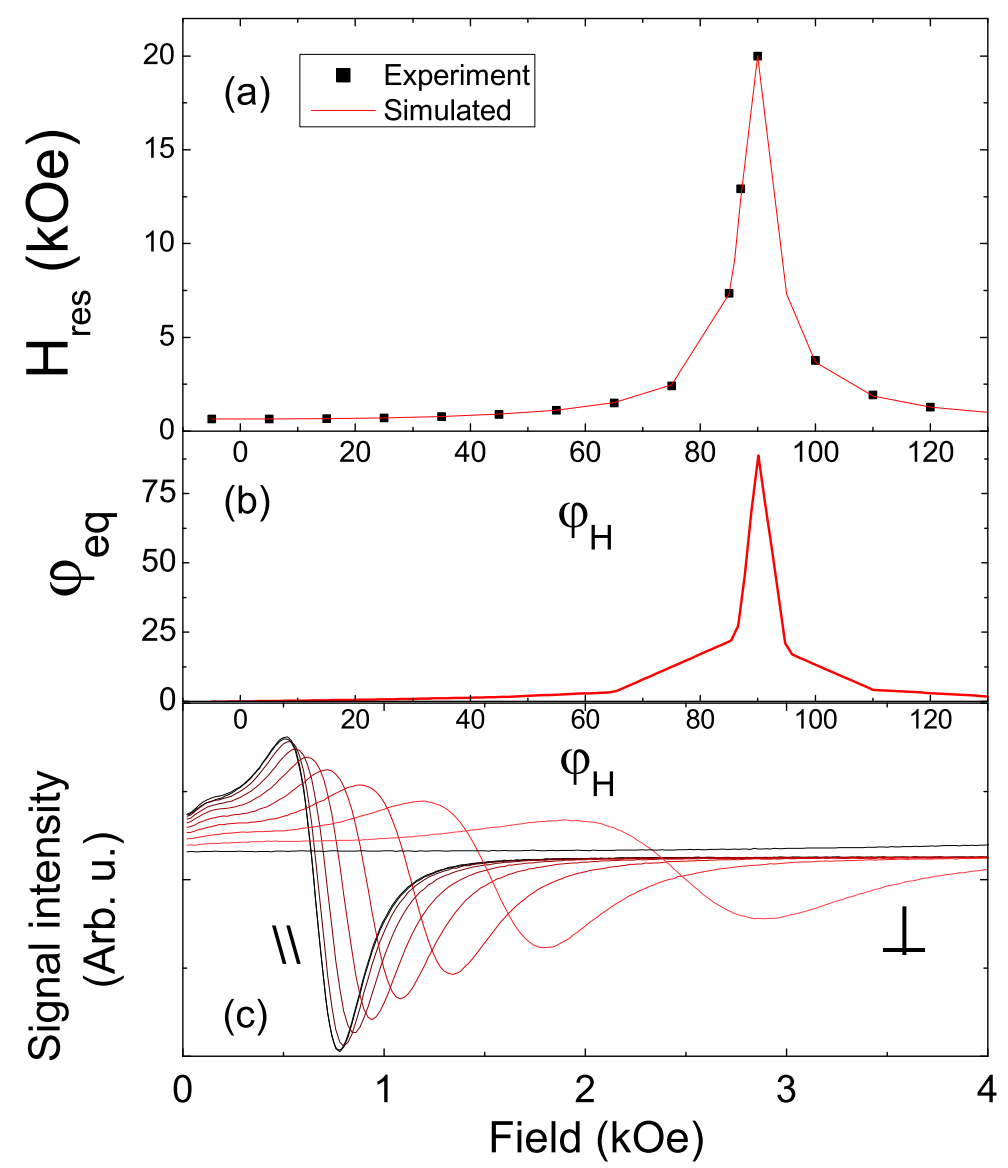

Figure 5.5: GdN24:(a) Resonance field as a function of the angle of the applied field out of the film plane. (b) $\phi_{e q}$ out of plane as a function of $\phi_{B}$ out of plane. (c) The raw signals showing resonance shift to higher fields as the sample is rotated. 
The angular dependence can be modelled as described in Section 2.1 and is shown in Fig. 5.5(a) as the solid red line. The magnetisation of the sample under resonance conditions must be known in order to start the modelling. In the present case resonance occurs at $4 \mathrm{~K}$ and approximately 600 Oe applied field in plane, and at $4 \mathrm{~K}$ and approximately 20000 Oe applied field out of plane. SQUID magnetometry was used to measure field loops in both geometries, and values of 1925 and $2075 \mathrm{emu} / \mathrm{cm}^{3}$ were determined. This sets the anisotropy constants to be used to simulate the full angular variation, and the good fit of the model to the experimental data indicates that the parameters used in the model are accurate. Cubic and uniaxial anisotropy constants are determined and are given in Table 5.2 .

Modelling of the angular dependence also allows for the determination of the angular variation of the magnetisation with respect to the film plane. Fig. 5.5(b) shows the angle of $\mathbf{M}$ relative to the film plane as a function of the angle of $\mathbf{H}$ relative to the film plane. In a bulk sample with no anisotropy, this plot would be linear, with the magnetisation always aligning itself along the direction of the applied field. This figure is a good illustration of how the anisotropy fields and demagnetising field act to align the magnetisation along directions other than that of the applied field.

Similar experiments were carried out on GdN26, a sample that differs from GdN24 only in that the nitrogen pressure during growth was a factor of two lower. The angular dependence is shown in Figure 5.6 and it is qualitatively very similar. The values of in plane and out of plane magnetisation under resonance conditions were 1860 and $1740 \mathrm{emu} / \mathrm{cm}^{3}$ respectively, determined by SQUID measurements. The anisotropy constants determined from the simulation are summarised in Table 5.2. Generally speaking, the similarity of the results for the two samples is evidence that the magnetisation of the two samples is subject to a similar crystalline environment and this is certainly in agreement with structural characterisation on these two samples. 
5.2. FERROMAGNETIC RESONANCE IN GDN

\begin{tabular}{|l|l|l|l|l|l|l|}
\hline $\mathrm{GdN}$ & $\begin{array}{l}\mathrm{M} \\
\left(\mathrm{emu} / \mathrm{cm}^{3}\right)\end{array}$ & $\begin{array}{l}4 \pi \mathrm{M} \\
(\mathrm{Oe})\end{array}$ & $\begin{array}{l}\mathrm{K}_{u} \\
\left(\mathrm{erg} / \mathrm{cm}^{3}\right)\end{array}$ & $\begin{array}{l}\mathrm{K}_{1} \\
\left(\mathrm{erg} / \mathrm{cm}^{3}\right)\end{array}$ & $\begin{array}{l}2 \mathrm{~K}_{u} / \mathrm{M} \\
(\mathrm{Oe})\end{array}$ & $\begin{array}{l}2 \mathrm{~K}_{1} / \mathrm{M} \\
(\mathrm{Oe})\end{array}$ \\
\hline 24 & 1925 & 24190 & $8.7 \times 10^{5}$ & $-1.6 \times 10^{5}$ & 910 & -160 \\
26 & 1860 & 23370 & $2.8 \times 10^{6}$ & $-1.5 \times 10^{4}$ & 3043 & -16 \\
$\mathrm{TED}^{1}$ & 1768 & 22220 & $5.1 \times 10^{6}$ & $1.1 \times 10^{6}$ & 5759 & 1292 \\
\hline
\end{tabular}

${ }^{1}$ Results for a polycrystalline thermal evaporation deposition grown sample from Ref.[56].

In order to interpret these results, we look back to our definition of the free energy (Equation 2.2). Easy axes are determined by minima in the free energy. The uniaxial term can be represented as $F_{u}=-K_{u} \cos ^{2} \theta$ where $\theta$ is the angle relative to the axis perpendicular to the film plane. Depending on the sign of $K_{u}$, this term will be minimised for $\theta=0$ or $\theta=\pi / 2$. For both films we find $K_{u}>0$ which implies there is an easy axis out of plane. Because the model of anisotropy is a phenomenological one, while it describes the anisotropy well, it cannot pinpoint the origin in this case. The sign of $K_{u}$ is consistent with previous studies on polycrystalline films although the values reported here are smaller [56]. This may indicate that is it shape effects rather than strain causing the anisotropy: epitaxial films are expected to be more strained than polycrystalline ones, and yet the anisotropy is found to be less. Additionally, XRD measurements indicate there is no detectable strain in the PLD films measured here. The larger coherence length and crystallite sizes in PLD films may be expected to reduce shape effects and hence the anisotropy.

The cubic magneto-crystalline anisotropy $K_{1}$ can be interpreted in the same way, where a negative value is associated with an easy axis along the [111] crystallographic direction [38]. The values for PLD films reported in Table 5.2 are not in agreement with those reported for polycrystalline films [56], shown in the third line of the table for reference. While the reason for this is not fully understood, it is expected that the results reported here on near single crystal films will give a more accurate picture of the cubic crystalline environment. The magnitude of the anisotropy field indicates this is a weak effect, but significant nonetheless as it is a result that may guide theory attempting to understand the origin of the magnetic ordering [29].

Further information can be extracted from the FMR linewidth, both as a func- 
tion of temperature and angle of applied field. There are two principle contributions to the linewidth: the intrinsic damping of the magnetisation precession, and broadening related to magnetic inhomogeneities [30]. The first term depends on the microwave frequency (constant in our measurements) and a damping factor which may exhibit angular anisotropy. However, it is reported that this first contribution to the linewidth is greatest when the magnetisation is near an easy axis $[30,57,58]$, which is quite different to what is observed in Figure 5.7, where the linewidth is greatest along the hard axis. Therefore we expect the second term is dominant in these samples. This term may in turn be separated into a contribution from some mosaicity of the sample, and a contribution from a distribution of internal fields in the sample: $H_{i}=4 \pi M+H_{1}+H_{u}$ where $H_{1}$ and $H_{u}$ are the cubic and uniaxial anisotropy fields respectively [59]. We find a good fit to the angular dependent linewidth with just the term representing a distribution of c-axes, which is of the form

$$
\Delta H=\Delta H_{0}+\Delta \phi_{H}\left(\frac{\partial H_{r e s}}{\partial \phi_{H}}\right)
$$

where $\Delta H_{0}$ is an intrinsic linewidth and $\Delta \phi_{H}$ is the angular spread of the c-axis. The best fit, shown in Figure 5.7(b), occurs for $\Delta \phi_{H}=2.5 \pm 0.5^{\circ}$, and this was determined by a least squares fitting procedure. We find good agreement with the distribution of c-axes in GdN determined by XRD rocking curves in section 2.4 , which was determined to be $2.8 \pm 0.2^{\circ}$. This implies the the c-axis spread is the dominant source of linewidth broadening, and justifies ignoring other contributions. 

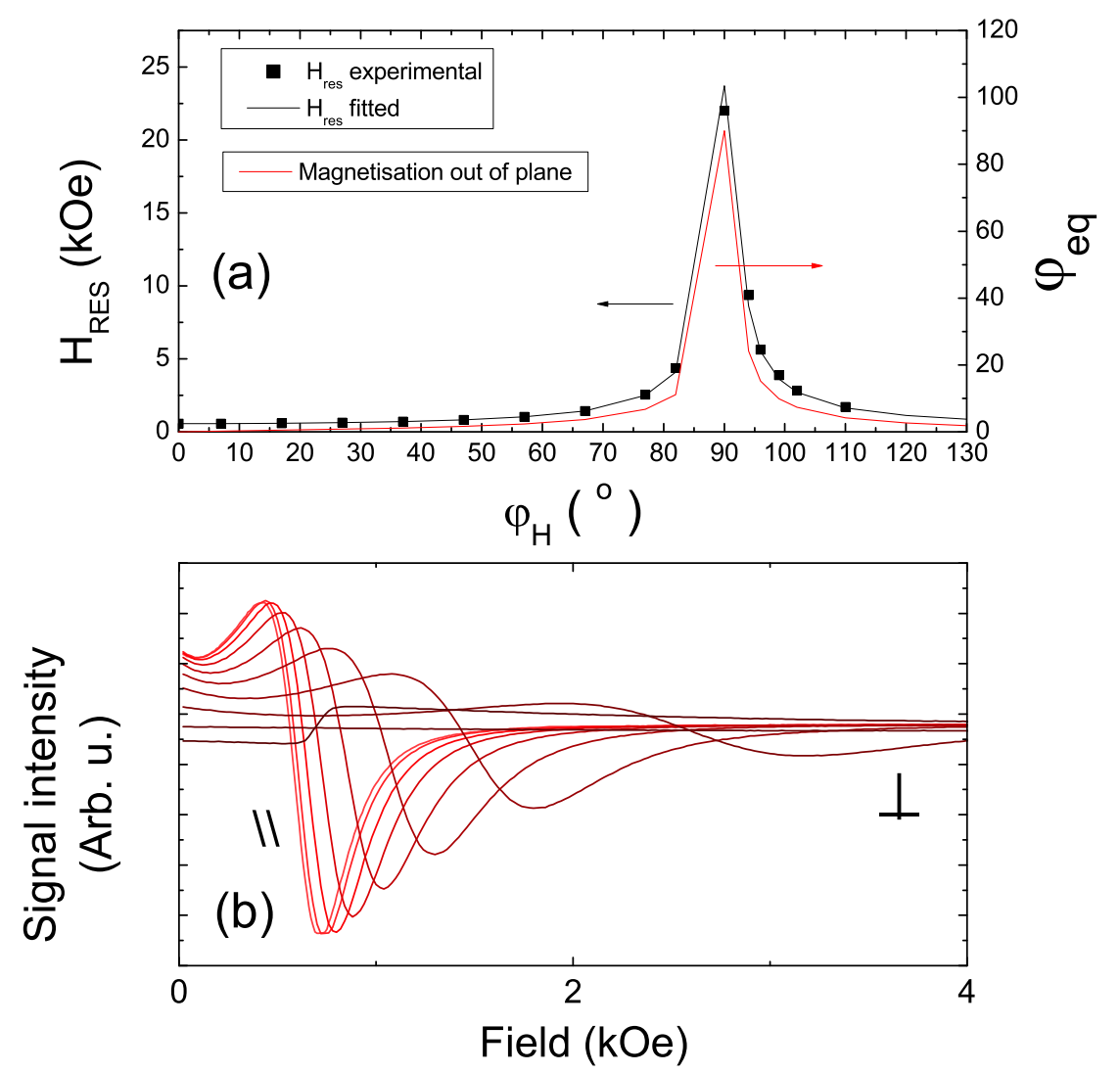

Figure 5.6: GdN26:(a) Resonance field as a function of the angle of the applied field out of the film plane. Experimental points, simulated line. The red line plots the magnetisation angle on the right hand axis. (b) The raw signals showing resonance shift to higher fields as the sample is rotated. (red - in plane, black - out of plane) 


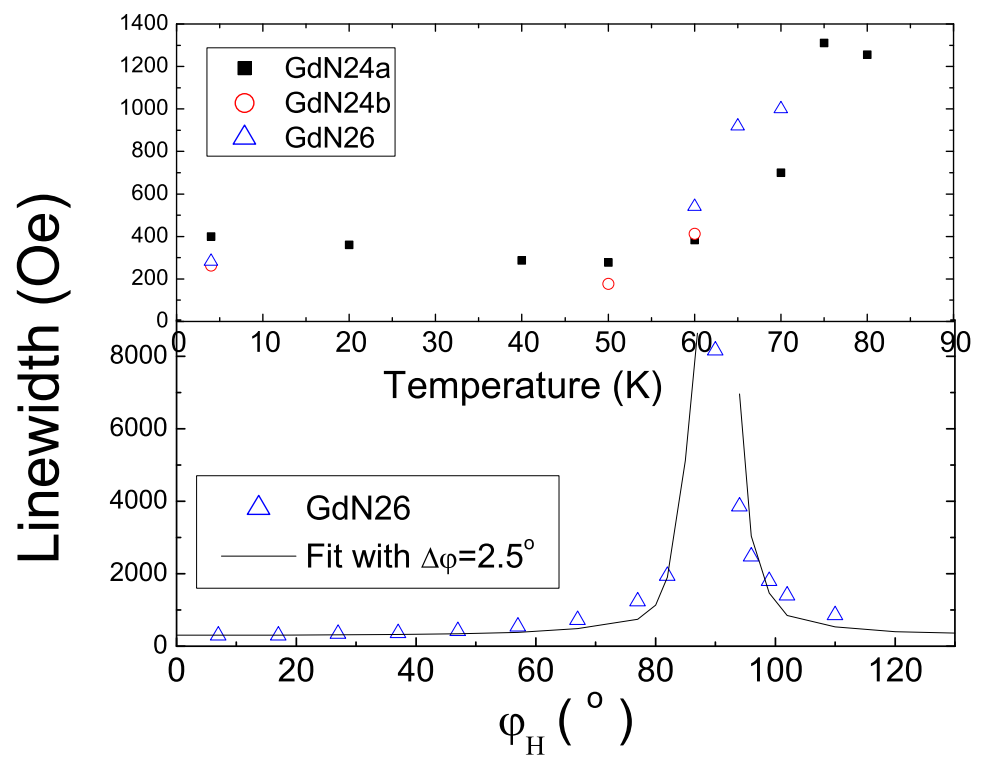

Figure 5.7: Resonance linewidth as a function of temperature, and linewidth as a function of the angle of the applied field fitted with Equation 5.1 for $\Delta \phi_{H}=2.5^{\circ}$. 


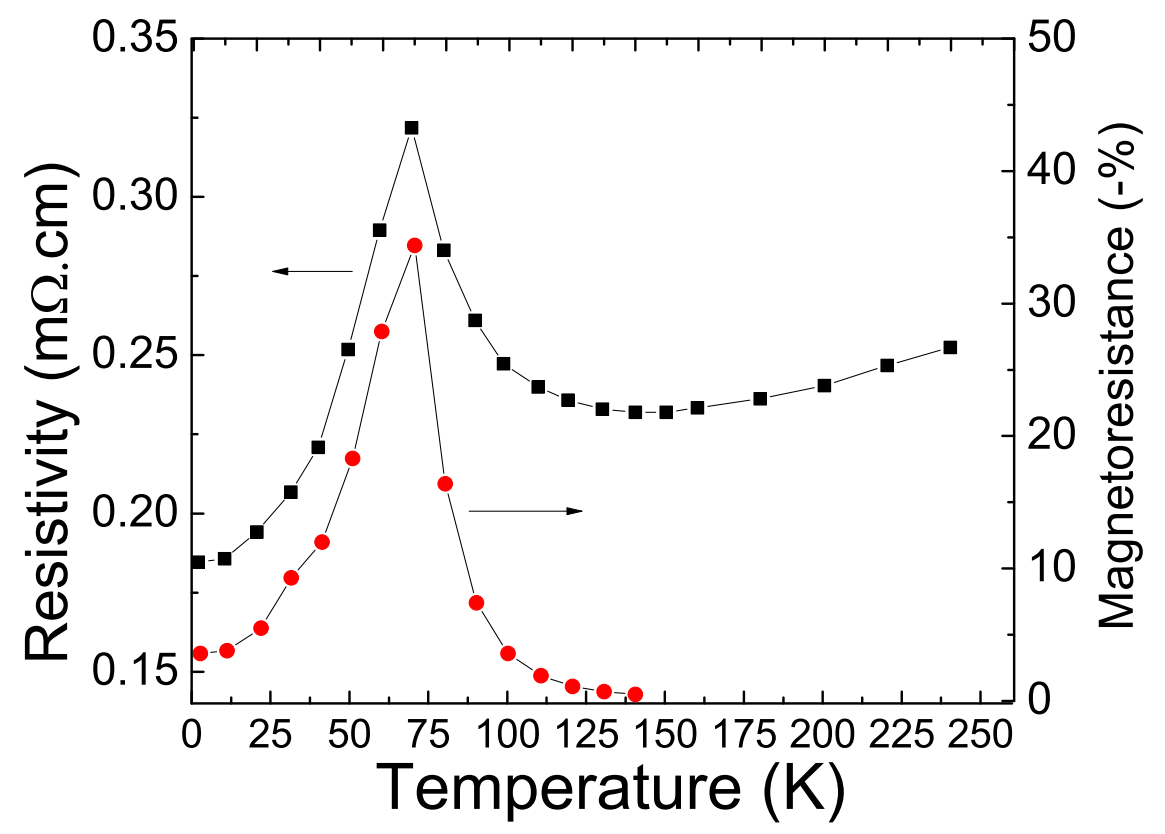

Figure 5.8: Temperature dependent resistivity and magnetoresistance for GdN07

\subsection{Magneto-transport in GdN}

Transport measurements indicate GdN grown by PLD has low resistivity. Figure 5.8 shows the temperature dependent resistivity typical of a PLD grown sample. We observe the peak in resistivity at the magnetic ordering temperature, in agreement with previously grown samples $[17,28]$. The resistivity decreases with temperature in the ferromagnetic region, finishing at a minimum value of $0.18 \mathrm{~m} \Omega \mathrm{cm}$ at $2 \mathrm{~K}$. There is no sign of the upturn in resistance at low temperature seen in samples with larger resistivity grown by other methods [28]. The peak in the resistivity is often attributed to a change in carrier concentration due to a closing of the band gap, occurring on top of a semiconducting background behaviour. An alternative explanation to be considered for the present films in which the peak in resistivity is not large relative to the overall resistivity is that the peak is due to an increase in magnetic scattering around the transition temperature due to magnetic disorder. We shall return to this discussion after looking at further 
results.

Magnetoresistance (MR), defined as $[\rho(0 \mathrm{~T})-\rho(4 \mathrm{~T})] / \rho(0 \mathrm{~T})$, is plotted with the resistivity in Figure 5.8. At high temperatures, application of a magnetic field has little or no effect on the resistivity. Around $\mathrm{T}_{C}$ the resistivity can be decreased by $30 \%$ as this is where the magnetic order can be significantly improved by the application of a magnetic field. For $\mathrm{T}<\mathrm{T}_{C}$, the sample is well ordered, and the application of a magnetic field once again has little affect on the transport properties. It should be noted that the magnetoresistance behaviour is consistent with either of the explanations of the peak in resistivity. It is clear that enhancing the ordering of the sample will suppress the peak if it is due to disorder. An external field would also have the effect of enhancing the effective transition temperature: the magnetic field 'helps' the exchange interaction overcome the thermal disorder at a higher temperature. Performing field cooled resistivity measurements would see the peak suppressed and shifted to higher temperatures, and this is indeed what is seen in these samples, as in Ref. [17]. 


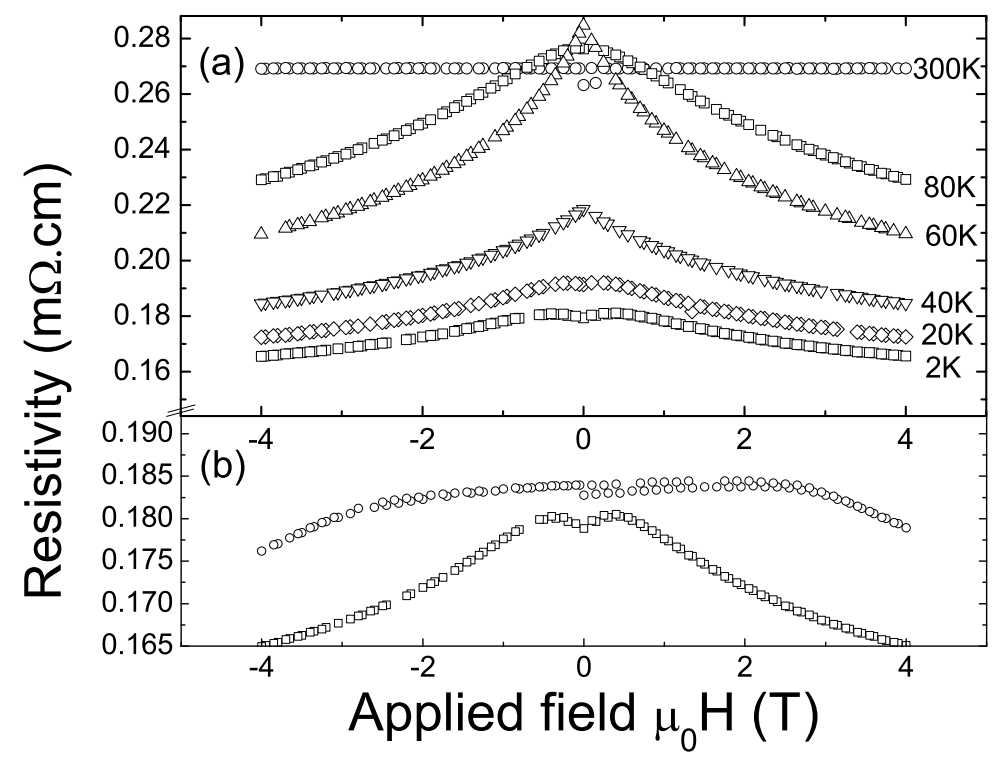

Figure 5.9: (a) In plane magnetoresistance for a range of temperatures. (b) In plane and out of plane MR at $2 \mathrm{~K}$. The in plane measurement shows the anomalous zero-field behaviour, while the out of plane measurement shows the effect of the demagnetising field. 
Magnetoresistance of sample GdN07 measured both with the field applied in the plane of the film, and perpendicular is shown in Figure 5.9. The ease with which the film is magnetised in plane is seen by the rapid change in resistivity with field. This can be compared to the same measurement done with the field applied perpendicular to the film plane (Fig. 5.9(b), circles), where the demagnetising field appears to retard the change in resistivity until it is saturated at about 2.7 T. At low fields applied in the film plane we observe an anomalous increase in the resistivity (Fig. 5.9(a), squares) which could be related to a small out of plane magnetisation as suggested by FMR above.

Hall effect measurements determine the carrier type and concentration. The negative gradient of the Hall resistance measured above $\mathrm{T}_{C}$ plotted against applied field in Figure 5.10(a) shows that the carriers are n-type, or electrons. There are two plausible sources of free electrons in these samples. If oxygen is substituted for a nitrogen atom in the rocksalt crystal structure, the 2- valence of the oxygen would leave a $5 \mathrm{~d}$ electron from $\mathrm{Gd}^{3+}$ in the conduction band. While there is some oxygen in the films at the substrate-film interface, this region is thought to form the bixbyite structure of $\mathrm{Gd}_{2} \mathrm{O}_{3}$ which is insulating. There will be some small region where there is substitutional oxygen, but if this were a significant factor, it would likely lead to a relatively large coercive field and poor magnetic saturation [60], and this is not observed. The second likely electron dopant is nitrogen vacancies. A single vacancy would leave up to three electrons in the conduction band. Just $1 \%$ nitrogen vacancies would suffice to explain the high carrier concentrations measured. The incorporation of nitrogen into the films is likely to be closely related to the preparation, which would explain the differences in transport properties between samples grown by different methods.

We observe the anomalous Hall effect (AHE) at temperatures nearing and below $\mathrm{T}_{C}$, as the magnetisation of the sample becomes significant. The Hall resistance in ferromagnets is generally expressed as

$$
R_{H}=\rho_{o} H+\rho_{a} \mu_{0} M
$$

where $\rho_{o}$ and $\rho_{a}$ are the ordinary and anomalous Hall coefficients, and $\mathrm{H}$ and $\mathrm{M}$ are the applied field and magnetisation [34]. The AHE contributes a Hall resistance proportional to the magnetisation, and the high-field slope returns the 


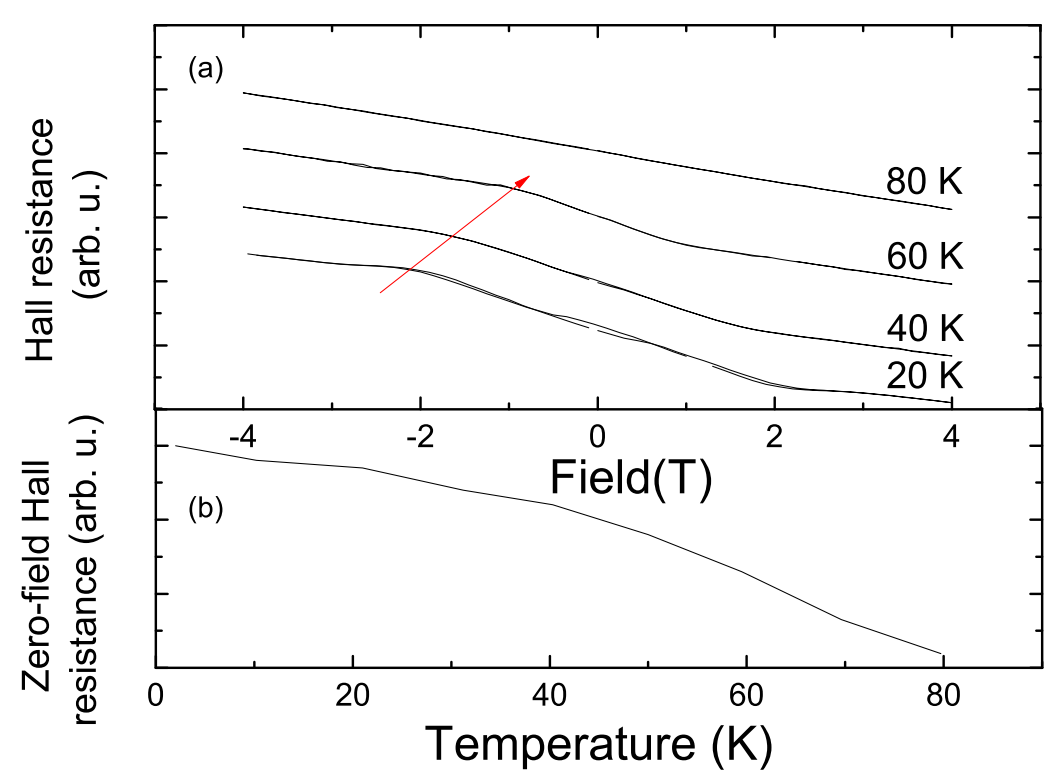

Figure 5.10: (a)Field dependent Hall resistance at a range of temperatures showing the onset of the anomalous Hall effect at $70 \mathrm{~K}$. (b)The zero field Hall resistance, extrapolated from the high field slope, resembles the magnetisation.

ordinary Hall coefficient, which is proportional to the carrier concentration and the applied field in the usual way. The anomalous and ordinary contributions can be seen in Figure 5.10(a). Extrapolating the high field slope to zero-field, and plotting this as a function of temperature we find something that resembles the magnetisation, as expected. This is plotted in Figure 5.10(b). The sharp kink in the Hall resistance curves of Fig. 5.10(a) show that the AHE term saturates completely with the magnetisation, and the field at which this occurs is equal to the demagnetising field, which is found to be in agreement with values determined by SQUID and MR.

The high-field gradient of the Hall resistance plotted as a function of applied field is proportional to the inverse carrier concentration as outlined in Section 3. At high field, the magnetisation is assumed to be saturated, and so the second term of Equation 5.3 is constant. The room temperature carrier concentration is 


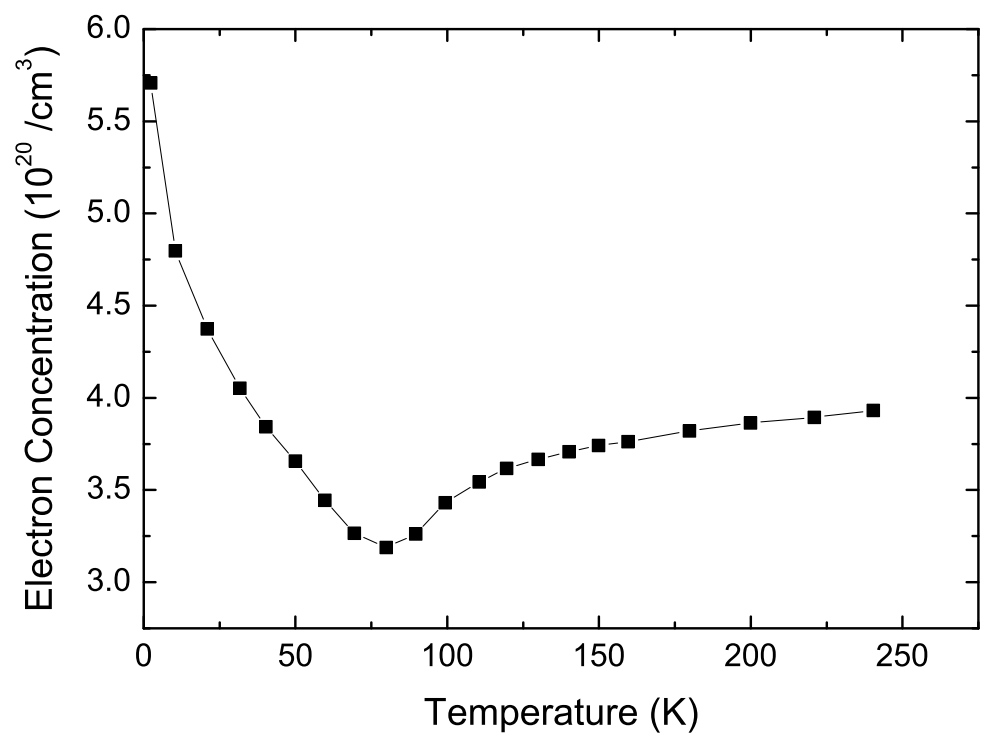

Figure 5.11: Carrier concentration plotted as a function of temperature. Carrier conc. has been extracted from the high field slope of Hall resistance measurements.

found to be $4 \times 10^{20} / \mathrm{cm}^{3}$ putting it on the boundary between a highly doped semiconductor and a metal. The carrier concentration is extracted and plotted as a function of temperature in Figure 5.11. The region around $\mathrm{T}_{C}$ must be treated with caution: the magnetisation may not be completely saturated at high field, meaning there could still be some contribution to the Hall slope from the anomalous Hall effect. If this is the case, then it is no longer accurate to associate the slope with the carrier concentration. Outside this uncertain region however, the initial dip around $100 \mathrm{~K}$ and the steep increase below $25 \mathrm{~K}$ are reliable, and constitute evidence of exchange splitting in the bands below $\mathrm{T}_{C}$. Moreover, the change in carrier concentration between room temperature and $2 \mathrm{~K}$ is about $40 \%$, the same as the drop in resistivity over this same temperature range. This indicates the resistivity changes are predominantly associated with a narrowing of the majority spin band gap and not with changes in the magnetic disorder induced scattering. 
The mobility of the electrons can be estimated to be $60 \mathrm{~cm}^{2} / \mathrm{V} . \mathrm{s}$ from the relation $\mu=1 / n e \rho$ where $\mathrm{n}$ is the carrier concentration, $\mathrm{e}$ is the electron charge, and $\rho$ is the resistivity. This is only an estimate as it assumes a single scattering time, where in reality there will be a band structure dependent distribution. The mean free path can also be estimated in a simple approximation using the free electron mass to be $11 \mathrm{~nm}$, in agreement with the crystallite sizes determined in Section 4.2.2. 



\section{Chapter 6}

\section{$\mathrm{SmN}$}

Chapter 4 has described the growth of samarium nitride. Samples grown were epitaxial and of high crystalline quality. The small magnetic moment of SmN complicates the magnetic measurements. Small corrections become significant and any impurities or sample defects can overwhelm the signal. Here we report on magnetisation measurements and FMR measurements, which both indicate the presence of $\mathrm{SmN}$, but detailed analysis was prevented by the presence of metallic Sm, present as a result of the PLD growth technique.

\subsection{Magnetometry in SmN}

The magnetisation of a number of SmN films were measured by SQUID magnetometry. A typical temperature dependent magnetisation measurement is shown in Figure 6.1. The total moment measured is very small and both FC and ZFC measured moments show an increase at low temperature. This behaviour, at first sight, seems paramagnetic with a $1 / \mathrm{T}$ dependence. However, there are some features not consistent with a purely paramagnetic description such as the splitting between the FC and ZFC curves. We have seen evidence suggesting the presence of metallic inclusions in these samples (see Section 4.2.4), and given the very small magnetic moment of $\mathrm{SmN}$ [21], we explore the possibility that the $\mathrm{SmN}$ signal is swamped by a paramagnetic signal from the metallic Sm inclusions.

Metallic Sm exists in two main phases. The first is a unique hexagonal struc- 
ture, repeating over nine layers, two thirds of which have hcp type nearest-neighbour environments, and one third having approximately fcc structure. This structure can be regarded as approximately rhombohedral [61]. The hexagonal sites order antiferromagnetically below $106 \mathrm{~K}$, and the cubic sites below $14 \mathrm{~K}$ [62]. A different, metastable hcp phase may be formed by quenching[63] and a study of its magnetic properties found it to be ferromagnetic below $160 \mathrm{~K}$ [61]. Both phases have been found to have surprisingly large paramagnetic susceptibilities, which are attributed to the mixing of the two lowest J multiplets $[64,65]$.

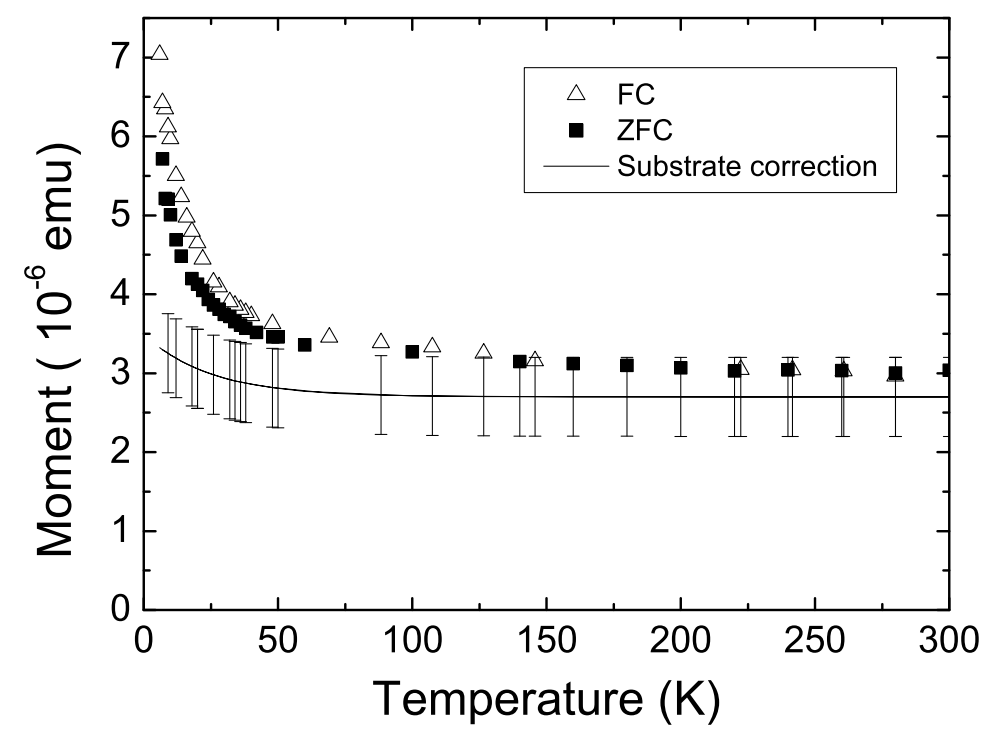

Figure 6.1: Uncorrected FC (triangles) and ZFC (squares) measurements of SmN05 in an applied field of 500 Oe. The solid line is the substrate and sample holder moment.

The importance of correcting the data for the substrate and other contributions is shown in Figure 6.1. A measurement of a substrate mounted using two polypropylene straws, one inside the other, gives a nearly temperature independent positive moment, with a slight increase below $50 \mathrm{~K}$. The origin of this moment must be dominated by substrate impurities and the sample holder, as YSZ is usually found to be diamagnetic, which would give a small negative temper- 
ature independent moment [66]. The small correction is only important because of the very small magnetic moments relating to SmN. Subtracting the correction and calculating the susceptibility, $\chi$, we might expect to see a significant positive, temperature independent term remain. This 'van Vleck susceptibility', $\chi_{0}$, has been observed in $\mathrm{SmN}$ and other $\mathrm{Sm}^{3+}$ compounds, including metallic samarium $[21,61,65]$. However, the Van Vleck susceptibility expected in $\mathrm{Sm}^{3+}$ compounds is on the order of $5 \times 10^{-4}$ which is smaller than the uncertainty in the substrate signal and so we cannot extract any information on the value of $\chi_{0}$.

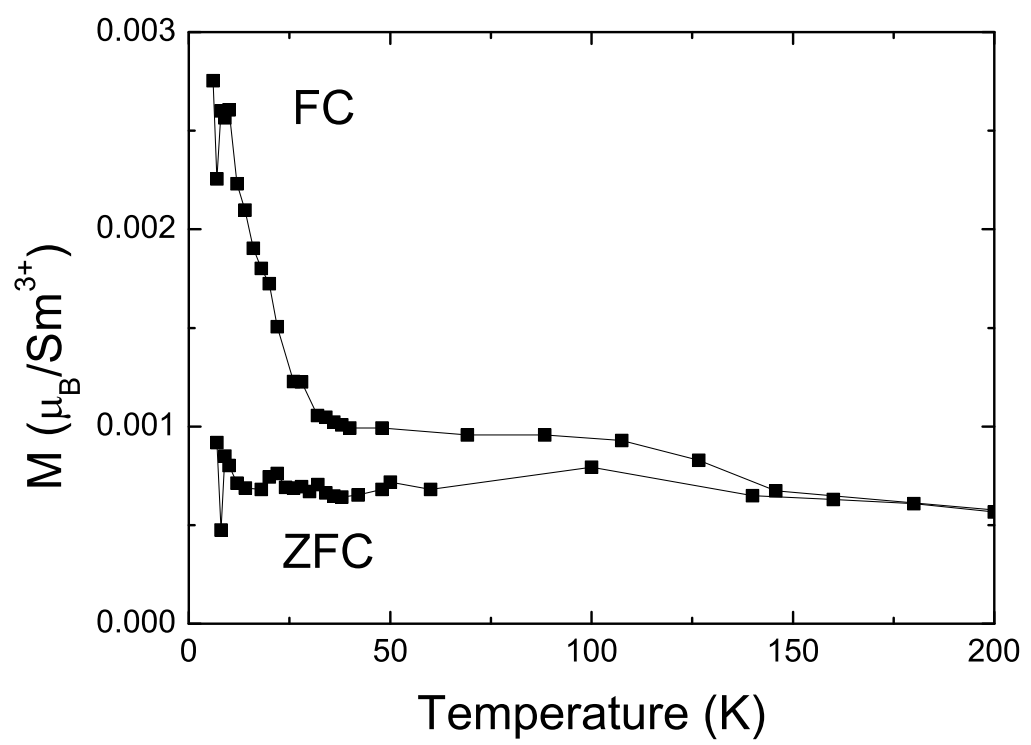

Figure 6.2: FC and ZFC magnetisation of SmN05 corrected for the substrate signal and a Curie like susceptibility.

The temperature dependent magnetisation appears to have a component that follows a Curie law. Figure 6.2 shows the result of subtracting a susceptibility of the form

$$
\chi=\chi_{0}+C / T,
$$

where $\chi_{0}$ is arbitrary as discussed above, but was chosen to leave a small constant susceptibility as would be expected for the $\mathrm{Sm}^{3+}$ ions in $\mathrm{SmN}$. $C$ was varied to minimise the $1 / T$ upturn in the ZFC data, while keeping all data points positive. 
Values of $C$ between 0.47 and $0.52 \mathrm{~K}$ achieved this. This Curie like behaviour is likely to be due to metallic $\mathrm{Sm}^{3+}$ in droplets in the films. The rhombohedral phase of Sm metal exhibits Curie behaviour down to approximately $10 \mathrm{~K}[64]$ where it becomes anti-ferromagnetic. Alternatively, the hcp phase, ferromagnetic below $160 \mathrm{~K}$, could give rise to superparamagnetism, which could explain the signal observed. This will be discussed in more detail below.

The magnetisation shown in Fig. 6.2 closely resembles the magnetisation of $\mathrm{SmN}$ reported recently [21]. A very weak ZFC signal reflects the large coercive field and the difficulty in saturating this material. The sharp upturn in the FC data are taken to signal the onset of ferromagnetic ordering at $30 \mathrm{~K} \pm 5 \mathrm{~K}$, in good agreement with the previous reference. The small moment is also in agreement with the quoted saturation magnetisation of $0.005 \mu_{B} / \mathrm{Sm}$ ion.

Another striking feature of Fig. 6.2 is the splitting of the FC and ZFC curves around $150 \mathrm{~K}$, which was not observed in the recently published SmN data. It has been shown that hcp samarium metal has a $\mathrm{T}_{C}$ of $160 \mathrm{~K}$ [61] so we conclude that there is some amount of the quenched hcp metallic Sm in the films. Noninteracting ferromagnetic clusters can behave like paramagnetic particles with a huge spin moment, a behaviour known as superparamagnetism. This provides a convincing explanation for the significant Curie-like signal in the raw data. The Curie constant is defined as

$$
C=\mu_{0} n \mu_{\mathrm{eff}}^{2} / 3 k_{\mathrm{B}}
$$

where $\mathrm{n}$ is the density of paramagnetic ions and $\mu_{\mathrm{eff}}$ is the effective moment of each ion. This can apply equally well to the superparamagnetic case where $n$ is the density of ferromagnetic clusters, and $\mu_{\mathrm{eff}}$ is the 'macrospin' of each cluster. Using the value of $C=0.5 \mathrm{~K}$ and the approximate density of droplets observed optically (see section 4.2.4) $n=10000 / \mathrm{mm}^{3}$ we find a macrospin of $130 \times 10^{6} \mu_{B}$ /droplet. Using a moment of $0.1 \mu_{B}$ per Sm ion in ferromagnetic hcp samarium $[61,65]$ we calculate the average number of ions per droplet and hence the average size of droplets, assuming a spherical shape. The resulting average droplet diameter is $700 \mathrm{~nm}$, in good agreement with the observed droplet size distribution of 100 to $3000 \mathrm{~nm}$. 


\subsection{Ferromagnetic resonance in $\mathrm{SmN}$}

FMR data were taken from a SmN sample of high crystalline quality, but with a droplet density estimated to be $10000 / \mathrm{mm}^{2}$. Spectra at a range of temperatures are shown in Figure 6.3(a). The dominant feature is the broad signal centred around $2500 \mathrm{Oe}$, although there are some other features near 1500 Oe that become apparent as the temperature is varied. We observe a small amount of angular dependence in the resonance field as shown in Fig. 6.3(b). The two data points at zero ${ }^{\circ}$ give an idea of the uncertainty in the resonance field. In stark contrast to $\mathrm{GdN}$, the angular dependence suggests that the demagnetising field is not the dominant source of anisotropy although the data could not be successfully fitted with the models used for GdN.

Figure 6.3(c) shows the temperature dependence of both the integrated signal intensity (which is proportional to the magnetisation) and the resonance field of the broad, dominant signals in Fig. 6.3(a). The magnetisation in black squares (left axis) shows a sharp increase around $8 \mathrm{~K}$, presumably signalling the onset of ferromagnetic ordering. The resonance field plotted as red triangles (right axis) tells a similar story: resonance shifts to higher fields as temperature increases, with a sharp increase around $6 \mathrm{~K}$. The resonance fields above $10 \mathrm{~K}$ are close to the value expected for a free electron, typical of a paramagnetic state. These measurements indicate that the material responsible for the broad signal in this sample has a Curie temperature of $7 \pm 1 \mathrm{~K}$.

Given the small value for $\mathrm{T}_{C}$ obtained here relative to previous reports, there is some question as to whether the material responsible for the observed signal is indeed SmN. SQUID data has shown that there are a number of contributions to the magnetic properties of the PLD films and it would be reasonable to ask whether the broad signal analysed in FMR might be due to one of these contributions. However, looking at spectra taken under similar conditions on a $\mathrm{SmN}$ film grown by thermal evaporation deposition (TED), there is evidence of a small resonance at a similar field. There are not likely to be any metallic inclusions in the TED films, although there could be a small amount of an oxide phase. This is not known to be ferromagnetic below any temperature thus the signal is most likely to originate from the $\mathrm{SmN}$, although the implications with regard to the 
magnetism of this material are not obvious. A more detailed analysis of FMR in a ferromagnetic material with a near-vanishing moment such as $\mathrm{SmN}$ would clearly be of significant interest. 

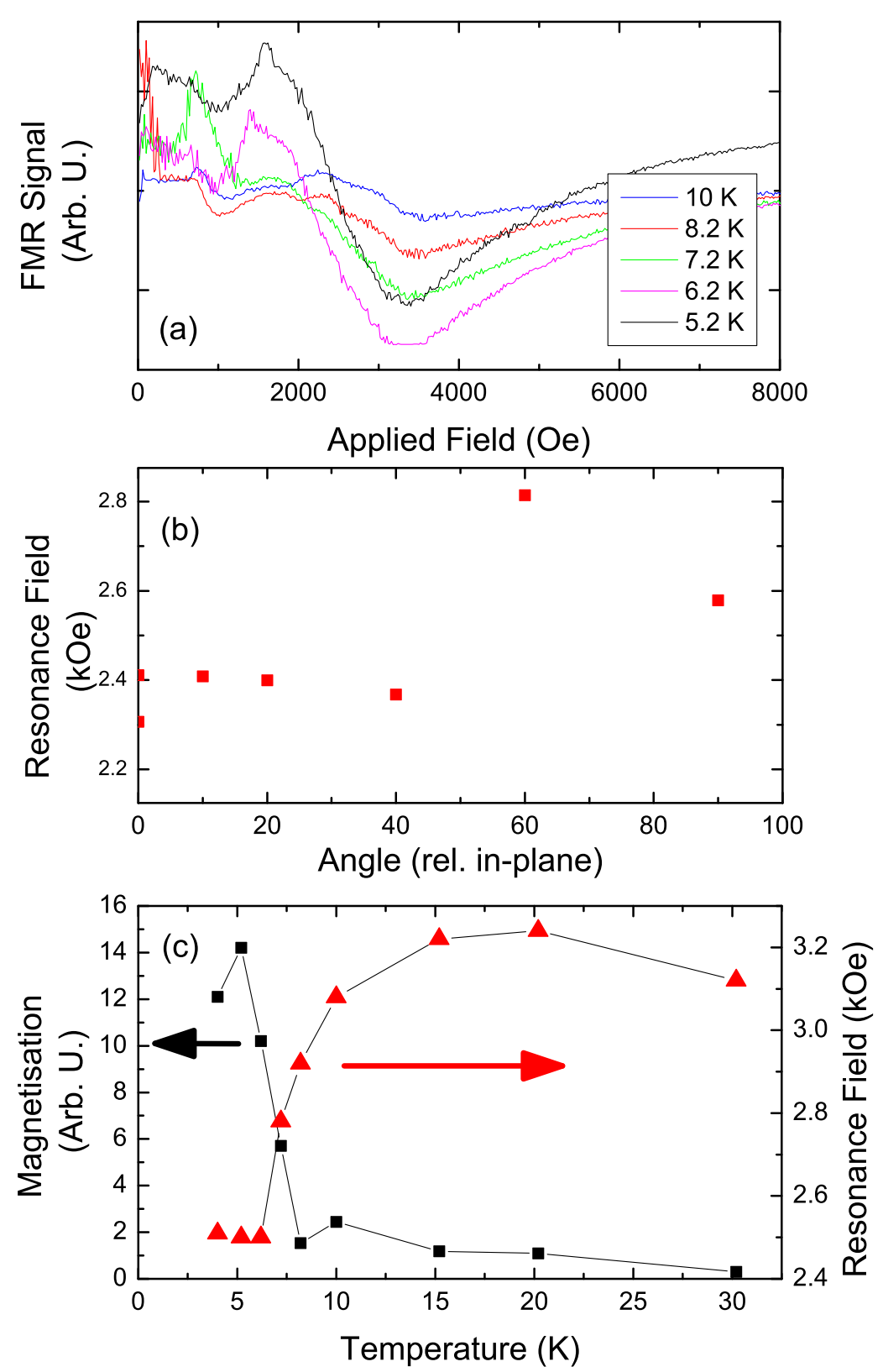

Figure 6.3: FMR results for SmN22. 



\section{Chapter 7}

\section{EuN preliminary investigation}

A small amount of work was done on europium nitride $(\mathrm{EuN})$ with 3 samples being grown by PLD. EuN is an interesting material in its own right and will undoubtedly be the subject of future studies. For this reason, the growth and preliminary measurements will be discussed here.

Eu sits between $\mathrm{Sm}$ and $\mathrm{Gd}$ in the rare earth series. $\mathrm{Eu}^{3+}$ has 6 electrons in the $4 f$ shell giving spin and orbital angular momentum quantum numbers $S=3$ and $L=3$ respectively. The $4 f$ shell being less than half full, Hund's rules tell us $S$ and $L$ align antiparallel to give $J=0$. The total moment of the ion is significant, given by $M=\mu_{B}|L+2 S| \approx 3 \mu_{B}$, but in the $J=0$ groundstate this will have zero projection along any axis [22]. This leads to the interesting situation of a strongly spin polarized but non-magnetic material.

The electronic structure is complicated by the tendency of $\mathrm{Eu}$ to form $\mathrm{Eu}^{2+}$ over $\mathrm{Eu}^{3+}$. LSDA+U calculations by Lambrecht et al. found solutions for both valencies, with the $2^{+}$valence predicted to be metallic and the $3^{+}$valence predicted to be half metallic [13]. Similar calculations by Horne et al. (SIC-LSDA) find EuN takes the trivalent state and is a half metal with a minority band gap of $1.4 \mathrm{eV}$ [67]. Questions relating to the valency, band structure, and magnetic properties of EuN can only be answered with experiments on defect and impurity free samples. To our knowledge, there have been few attempts at making high quality EuN samples, and even fewer detailed studies of the magnetic or electronic properties of reliable EuN samples. 


\subsection{Sample preparation}

Eu metal is a highly reactive substance and is stored under oil. The target was extensively degreased before being loaded into the PLD growth chamber. After a standard outgassing procedure, there was no evidence of organics or any other impurities at levels above what is usually detected.

Three EuN samples were grown by PLD on YSZ [100] substrates. Growth parameters were partly chosen based on our experience with samarium and gadolinium. Europium has a lower melting point and higher vapour pressure than both Sm and Gd and was therefore expected to be 'easier' to ablate [51]. 'Easier' in this case meaning a lower ablation threshold, or a higher atomic flux for an equivalent fluence compared to Sm or Gd. Based on this the fluence chosen was on the lower end of the range we have used.

There has been a lot of work done on the growth of europium oxide $(\mathrm{EuO})$ and this may also be used to guide the growth of EuN. Steeneken et al. looked at the role of substrate temperature in film growth [68]. They report that at substrate temperatures above $340^{\circ} \mathrm{C}$ metallic Eu is re-evaporated from the surface while for lower substrate temperatures metallic Eu layers begin to form. This value was determined for pure Eu evaporation, in the absence of oxygen. It may therefore apply equally well to our nitride growths as to their oxide growths. This gave us a lower limit for the substrate temperature; films were grown at $600^{\circ} \mathrm{C}, 500^{\circ} \mathrm{C}$, and $400^{\circ} \mathrm{C}$, all in the regime where it was expected excess Eu would be re-evaporated. Some of the growth parameters are outlined in table 7.1.

\begin{tabular}{|c|l|c|}
\hline Growth type and ID & $\begin{array}{l}\text { Substrate/Film growth time } \\
\text { (min)/Cap/Cap growth time } \\
(\mathrm{min})\end{array}$ & $\mathrm{T}_{\text {subs }}\left({ }^{\circ} \mathrm{C}\right)$ \\
\hline PLD EuN1 & $\mathrm{YSZ} / 60 / \mathrm{Cr} / 30$ & 600 \\
PLD EuN2 & $\mathrm{YSZ} / 120 / \mathrm{YSZ} / 30$ & 400 \\
PLD EuN3 & $\mathrm{YSZ} / 30 / \mathrm{YSZ} / 15$ & 500 \\
\hline
\end{tabular}




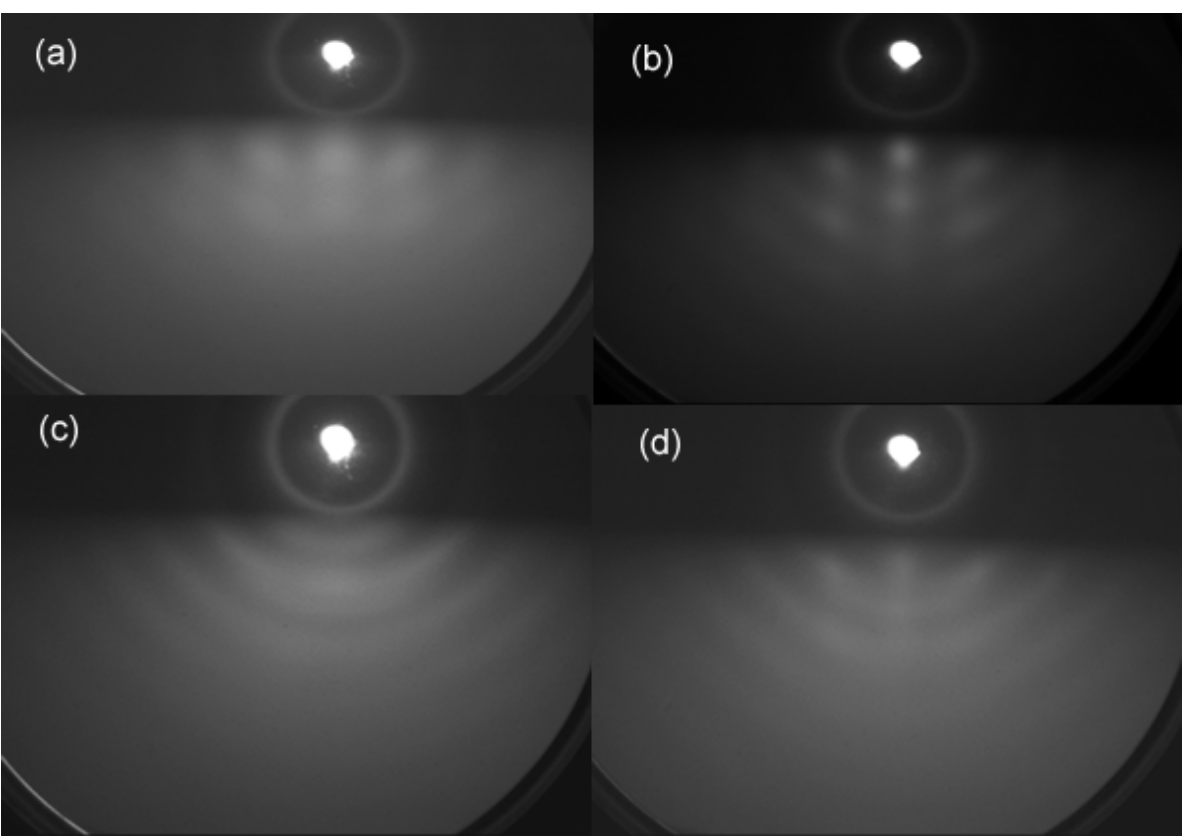

Figure 7.1: (a) EuN1 @ 5 mins. (b) EuN2 @ 5 mins. (c) EuN1 @ 30 mins. (d) EuN2@ 30 mins.

\subsection{Characterisation}

In situ RHEED from the three samples grown by PLD indicate the samples are polycrystalline. Figures 7.1(a) and (b) show samples 1 and 2 having some degree of in-plane texturing in the first five minutes of growth. Crystal quality then continued to deteriorate and by 30 minutes of growth, both samples were completely polycrystalline as evidenced in Figs. 7.1(c) and (d). The growth of EuN3, with an intermediate growth temperature of $500^{\circ} \mathrm{C}$ was qualitatively similar.

The RHEED patterns seen in the first few minutes of growth do allow an analysis of the relaxation behaviour, although the uncertainties are large given the difficulty in consistently extracting peak positions from such a disordered pattern. There is a rapid change in the surface lattice spacing from that of the YSZ substrate in both cases. EuN1 shows similar behaviour to what was observed for $\mathrm{GdN}$ and $\mathrm{SmN}$, the spacing peaking at $5.4 \AA$ then returning towards the bulk value. The lattice relaxes to about $5.16 \AA$ after just over a minute of growth 
and then gradually increases to the 5 minute mark, where the spacing could no longer be determined. It should be noted that the measurement error, although not quantified, is expected to increase with growth time given the increasing difficulty in extracting a lattice parameter. The large FWHM of EuN1 reflects the poor crystalline quality. EuN2, grown at $400{ }^{\circ} \mathrm{C}$, is seen to relax at a significantly larger lattice parameter than seen for EuN1, despite the RHEED appearing qualitatively similar (i.e. polycrystalline cubic material on a cubic substrate).

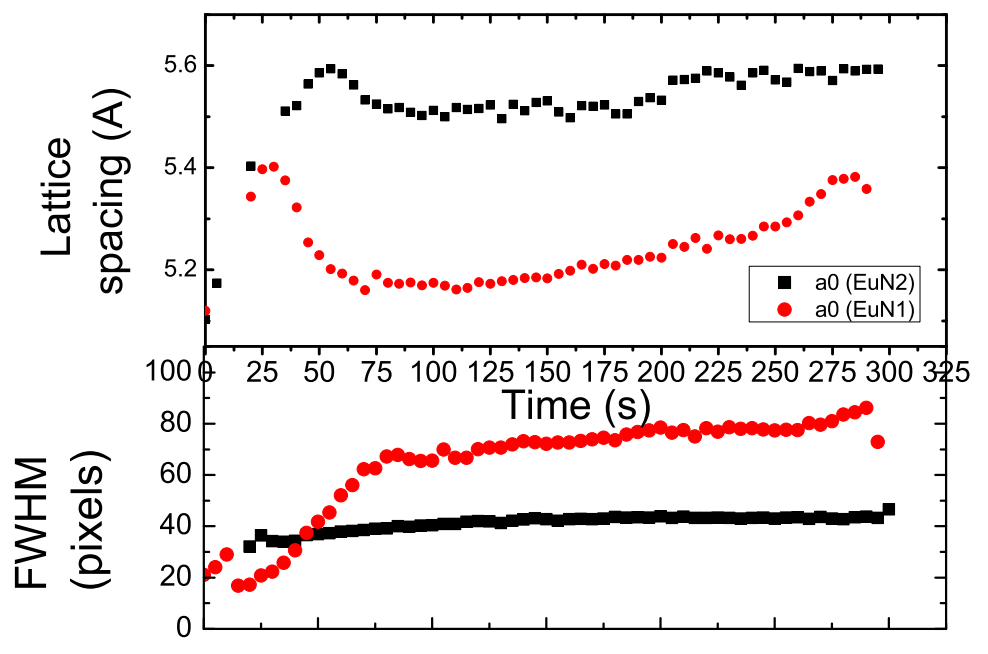

Figure 7.2: The in plane lattice spacing and FWHM of the central streak as a function of time for two EuN growths.

XRD on EuN2 and EuN3 strongly indicate some significant differences between these two samples. Figure 7.3 shows $\theta-2 \theta$ scans with the main peaks labelled. EuN2 shows none of the peaks expected for EuN, but rather the peaks associated with metallic europium. EuN3 on the other hand, has a strong reflection only at $30.79^{\circ}$, corresponding to the EuN [111] line; EuN3 is [111] textured EuN with a c-axis lattice parameter of $5.03 \AA \pm 0.01 \AA$, in good agreement with theoretical values [22].

It is interesting to relate the information gleaned from RHEED and XRD to the sample preparation. RHEED shows two different in-plane lattice spacings 


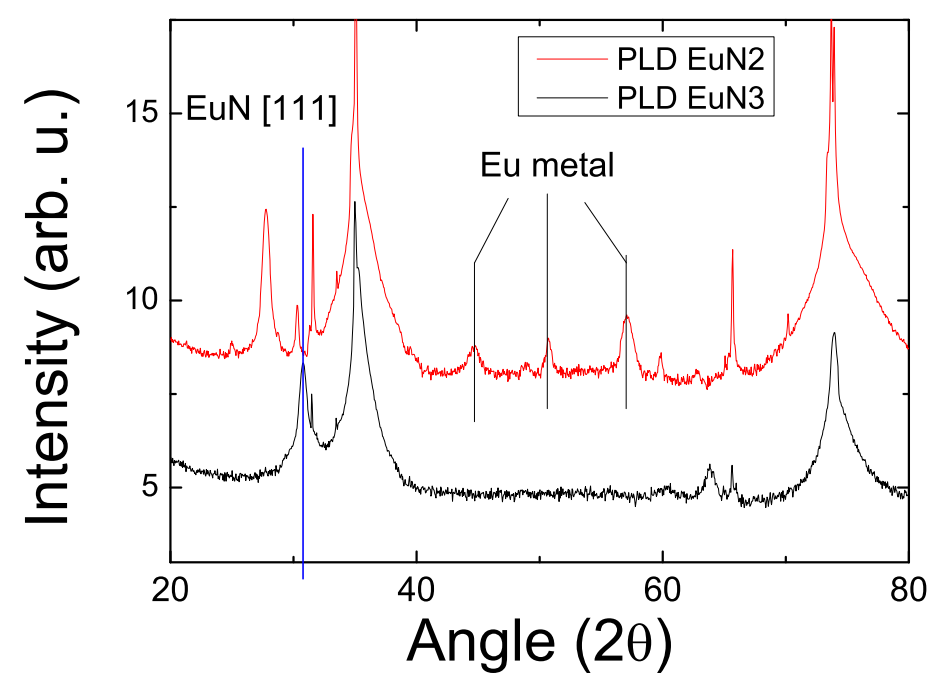

Figure 7.3: XRD on two EuN samples reveals EuN2 has metallic Eu phases, while EuN3 appears to be [111] oriented EuN.

for EuN1 and EuN2, and XRD explains this with evidence that the sample optimistically named EuN2 is in fact metallic Eu. XRD also confirms that EuN3 is textured EuN and so it is inferred that EuN1 is similar (XRD data could not be obtained for this sample). EuN2 was grown at $400{ }^{\circ} \mathrm{C}$, while the growths that produced polycrystalline EuN were done at 500 and $600^{\circ} \mathrm{C}$. It was mentioned above that Steeneken et al. found excess europium could be re-evaporated for substrate temperatures above $350{ }^{\circ} \mathrm{C}$ in a MBE system. It is plausible that given the not insignificant differences in growth methods (PLD vs MBE), namely the higher atomic flux in the PLD method, the temperature required for re-evaporating excess Eu metal is higher in the PLD system. This is a possible explanation for why EuN2 is metallic Eu, while the samples grown at slightly higher temperature are europium nitride.

The main conclusion to draw from this is that EuN can be grown by PLD at temperatures above $400^{\circ} \mathrm{C}$. While the first few minutes of growth are epitaxial, the sample quickly becomes polycrystalline in-plane, and [111] textured out of plane. The observation of the correct lattice spacing in both XRD and RHEED 
confirms the presence of EuN, which means the europium takes a 3+ valency answering an open question from a theoretical point of view. 


\section{Chapter 8}

\section{Conclusion}

The growth technique of pulsed laser deposition, novel in its application to rare earth nitride growth, has been used to grow epitaxial GdN, SmN and EuN on (001) oriented YSZ. Structural characterisation indicates the first two materials are of high crystalline quality, while EuN is found to be polycrystalline after the first few minutes of growth.

The growth temperature has been optimised for $\mathrm{SmN}$ based on the surface coherence length obtained from RHEED. It is found to be $600 \pm 100^{\circ} \mathrm{C}$ which is related to the temperature at which excess samarium can be re-evaporated from the surface. A similar result is seen for EuN where growths at 500 and $600{ }^{\circ} \mathrm{C}$ produced EuN, while a $400{ }^{\circ} \mathrm{C}$ growth appeared to contain mostly metallic Eu phases.

Metallic rare earth droplets were observed in these films. These are a result of the PLD growth process and are difficult to avoid. The effect of the droplets was most pronounced in $\mathrm{SmN}$ where the magnetic moment of the nitride is of a similar order of magnitude to the moment due to the droplets. A comparison of FMR results for GdN films indicates that the number of droplets was successfully reduced by shorter growth times and growing with a larger spot area did not seem to significantly increase the number of droplets. The conclusion is that ablating with a large spot size for a shorter period of time will minimise the number of droplets in a film.

GdN was found to have what appears to be a magnetically 'dead' layer. The 
thickness of the sample based on RBS measurements was always up to $40 \mathrm{~nm}$ larger than the thickness implied by the magnetisation. This is consistent with the interfacial oxide layer observed by RHEED which is expected to be nonmagnetic. A similar effect is expected in $\mathrm{SmN}$ and EuN, although it was observed only by RHEED and not by magnetic measurements. Understanding this early growth behaviour will be an important step towards improving both the crystal quality and the magnetic properties of the films.

The GdN films grown by PLD all have a similar Curie temperature of $70 \mathrm{~K}$ with a magnetisation that saturates at $7 \mu_{B} / \mathrm{Gd}$ ion to within an uncertainty dominated by the volume of the film. The consistency of $\mathrm{T}_{C}$ is remarkable given the variation in growth parameters in the PLD growth. Even more remarkable is that the same $\mathrm{T}_{C}$ has now been reported for samples varying in carrier concentration by 4 orders of magnitude, which implies the ferromagnetic exchange interaction is not mediated by the conduction electrons.

Coercive fields as small as 10 Oe are reported in $\mathrm{GdN}$, and it is shown that the coercive field is inversely proportional to the coherence length in the crystal, which is related to dislocations and grain boundaries. The present work has shown that the relationship exists, but has been unable to show how to systematically control the coercive field through the growth parameters. With advances in film growth, it should become possible to introduce dislocations in a controlled manner and hence tune the coercive field.

The magnetisation orientation is overwhelmingly 'easy-in-plane' type due to the strong demagnetising field of the thin films. A small uniaxial anisotropy also exists due to either shape anisotropy or strain. An interesting result is the small magnetocrystalline anisotropy that shows an easy axis along [111]. As well as being of interest in terms of the theoretical origins of magnetism in this material, it may explain the zero-field anomaly observed in the magnetoresistance.

Hall effect measurements establish that the carriers are electrons and show the carrier concentration to be on the order of $10^{20}$ electrons $/ \mathrm{cm}^{3}$. This is likely to be due to electron doping by nitrogen vacancies, and the temperature dependent resistivity behaves like that of a degenerately doped semiconductor. Following the carrier concentration as a function of temperature, we report evidence of the bands shifting below $\mathrm{T}_{C}$ due to exchange splitting. 
Magnetic measurements on $\mathrm{SmN}$ were dominated by the metallic inclusions resulting from the growth technique. Field-cooled measurements show these metallic droplets to be ferromagnetic below $160 \mathrm{~K}$, a feature of melt-quenched samarium metal. Superparamagnetism of the ferromagnetic droplets is presented as the likely origin of the large Curie-like temperature dependence of the susceptibility at low temperature. Given the sensitivity of the magnetic properties of $\mathrm{SmN}$ to the presence of droplets, PLD is not the optimal growth technique for determining these magnetic properties. PLD has however, led to the optimisation of certain growth parameters such as temperature that can be used to grow quality SmN films by other methods. PLD SmN films may also be suitable for transport measurements.

In summary, epitaxial thin films of $\mathrm{GdN}, \mathrm{SmN}$, and EuN have been grown by PLD. Investigations of these films have led to an elucidation of the magnetic and transport properties of these rare earth nitrides. 



\section{Bibliography}

[1] D. D. Awschalom and M. E. Flatte, Challenges for semiconductor spintronics, Nature Physics 3, 153 (2007).

[2] S. Bandyopadhyay and M. Cahay, Introduction to spintronics (CRC Press) (2008).

[3] I. Zutic, J. Fabian, and S. D. Sarma, Spintronics: Fundamentals and applications, Rev. Mod. Phys. 76, 323 (2003).

[4] E. L. Nagaev, Colossal Magnetoresistance Materials and Other Magnetic Semiconductors (World Scientific Publishing Company) (2002).

[5] B. J. Ruck, Spintronics potential of rare-earth nitrides and related materials (2009).

[6] T. S. Santos, J. S. Moodera, K. V. Raman, E. Negusse, J. Holroyd, J. Dvorak, M. Liberati, Y. U. Idzerda, and E. Arenholz, Determining exchange splitting in a magnetic semiconductor by spin-filter tunneling, Phys. Rev. Lett. 101, 147201 (2008).

[7] G. Busch, Magnetic properties of rare-earth compounds, Journal of Applied Physics 38, 3 (1967).

[8] F. Hulliger, Handbook on the Physics and Chemistry of Rare Earths Vol. 4 (NorthHolland Physics Publishing, New York) (1979).

[9] R. A. Cutler and A. W. Lawson, Synthesis and magnetic behavior of GdN, Journal of Applied Physics 46, 2739 (1975). 
[10] P. Wachter and E. Kaldis, Magnetic interaction and carrier concentration in GdN and $\mathrm{GdN}_{1 ? x} \mathrm{O}_{x}$, Solid State Commun. 34, 4 (1980).

[11] W. R. L. Lambrecht, Electronic structure and optical spectra of the semimetal ScAs and of the indirect-band-gap semiconductors ScN and GdN, Phys. Rev. B 62, 20 (2000).

[12] P. Larson and W. R. L. Lambrecht, Electronic structure of Gd pnictides calculated within the LSDA + U approach, Phys. Rev. B 74, 085108 (2006).

[13] P. Larson, W. R. L. Lambrecht, A. Chantis, and M. van Schilfgaarde, Electronic structure of rare-earth nitrides using the LSDA +U approach: Importance of allowing 4 f orbitals to break the cubic crystal symmetry, Phys. Rev. B 75, 045114 (2007).

[14] C. M. Aerts, P. Strange, M. Horne, W. M. Temmerman, Z. Szotek, and A. Svane, Half-metallic to insulating behavior of rare-earth nitrides, Phys. Rev. B 69, 045115 (2004).

[15] C. G. Duan, R. F. Sabirianov, W. N. Mei, P. A. Dowben, S. S. Jaswal, and E. Y. Tsymbal, Electronic, magnetic and transport properties of rare-earth monopnictides, J. Phys.: Condens. Matter 19, 315220 (2007).

[16] C. G. Duan, R. F. Sabiryanov, J. Liu, W. N. Mei, P. A. Dowben, and J. R. Hardy, Theoretical study of the magnetic ordering in rare-earth compounds with face-centered-cubic structure, J. Appl. Phys. 97, 10A915 (2005).

[17] F. Leuenberger, A. Parge, W. Felsch, K. Fauth, and M. Hessler, GdN thin films: Bulk and local electronic and magnetic properties, Phys. Rev. B 72, 014427 (2005).

[18] J. W. Gerlach, J. Mennig, and B. Rauschenbach, Epitaxial gadolinium nitride thin films, Appl. Phys. Lett. 90, 061919 (2007).

[19] M. Scarpulla, C. Gallinat, S. Mack, J. Speck, and A. Gossard, GdN (111) heteroepitaxy on GaN (0001) by N2 plasma and NH3 molecular beam epitaxy, Journal of Crystal Growth 311, 5 (2009). 
[20] A. R. H. Preston, S. Granville, D. H. Housden, B. Ludbrook, B. J. Ruck, H. J. Trodahl, A. Bittar, G. V. M. Williams, J. E. Downes, K. E. Smith, Y. Zhang, A. DeMasi, and W. R. L. Lambrecht, Comparison between experiment and calculated band structures for DyN and SmN, Phys. Rev. B 76, 245120 (2007).

[21] C. Meyer, B. J. Ruck, J. Zhong, S. Granville, A. R. H. Preston, G. V. M. Williams, and H. J. Trodahl, Near-zero-moment ferromagnetism in the semiconductor SmN, Phys. Rev. B 78, 174406 (2008).

[22] M. D. Johannes and W. E. Pickett, Magnetic coupling between nonmagnetic ions: $E u^{3+}$ in EuN and EuP, Phys. Rev. B 72, 195116 (2005).

[23] A. Hasegawa and A. Yanase, Energy band structures of Gd-pnictides, J. Phys. Soc. Jpn. 42, 492 (1977).

[24] C. G. Duan, R. F. Sabiryanov, J. Liu, W. N. Mei, P. A. Dowben, and J. R. Hardy, Strain induced half-metal to semiconductor transition in GdN, Phys. Rev. Lett. 94, 237201 (2005).

[25] A. N. Chantis, M. van Schilfgaarde, and T. Kotani, Quasiparticle self-consistent GW method applied to localized 4 felectron systems, Physical Review B (Condensed Matter and Materials Physics) 76, 165126 (2007).

[26] A. R. H. Preston, Ph.D. thesis, Victoria University of Wellington (2009).

[27] H. J. Trodahl, A. R. H. Preston, J. Zhong, B. J. Ruck, N. M. Strickland, C. Mitra, and W. R. L. Lambrecht, Ferromagnetic redshift of the optical gap in GdN, Phys. Rev. B 76, 085211 (2007).

[28] S. Granville, B. J. Ruck, F. Budde, A. Koo, D. J. Pringle, F. K. amd A. R. H. Preston, D. H. Housden, N. Lund, A. Bittar, G. V. M. Williams, and H. J. Trodahl, Semiconducting ground state of GdN thin films, Phys. Rev. B 73, 235335 (2006).

[29] C. Mitra and W. R. L. Lambrecht, Magnetic exchange interactions in the gadolinium pnictides from first principles, Phys. Rev. B 78, 134421 (2008).

[30] M. Farle, Ferromagnetic resonance of ultrathin metallic layers, Rep. Prog. Phys. 61, 755 (1998). 
[31] J. Smit and H. Beljers, Philips Res. Rep. 10, 113 (1955).

[32] K. Gramm, L. Lundgren, and . Beckman, SQUID magnetometer for magnetization measurements, Physica Scripta. 13, 93 (1976).

[33] C. L. Chien and C. R. Westgate (editors), The Hall Effect and its Applications (Plenum, New York) (1980).

[34] H. Toyosaki, T. Fukumura, Y. Yamada, K. Nakajima, T. Chikyow, T. Hasegawa, H. Koinuma, and M. Kawasaki, Anomalous hall effect governed by electron doping in a room-temperature transparent ferromagnetic semiconductor, Nature materials 3, 221 (2004).

[35] A. S. Arrot, Ultrathin Magnetic Structures 1 (Springer) (2005).

[36] C. Kittel, Introduction to Solid State Physics (Wiley) (1986).

[37] N. W. Ashcroft and N. D. Mermin, Solid State Physics (Thomson) (1976).

[38] R. Skomski, Simple Models of magnetism (Oxford University press) (2008).

[39] E. du T. de Lacheisserie (editor), Magnétisme (EDP Sciences) (2000).

[40] W. J. M. de Jonge, P. J. H. Bloemen, and F. J. A. den Broeder, Ultrathin magnetic structures I (Springer), chap. Experimental investigations of magnetic anisotropy, p. 65 to 90 (2005).

[41] G. H. O. Daalderop, P. J. Kelly, and M. F. H. Schuurmans, Ultrathin magnetic structures I (Springer), chap. Magnetic anisotropy from first principles, p. 40 to 65 (2005).

[42] S. E. Granville, Structural, electronic and magnetic properties of GaMnN, MnN and rare-earth nitride thin films, Ph.D. thesis, Victoria University of Wellington (2007).

[43] R. K. Singh and J. Narayan, Pulsed-laser evaporation technique for deposition of thin films: Physics and theoretical model, Phys. Rev. B 41, 8843 (1990). 
[44] F. Robaut, Elaboration par depot laser pulse at caracterisation de couches minces epitaxiees de metaux de terres rares et de composes intermetalliques terre rare-cobalt, Ph.D. thesis, Louis Neel, C.N.R.S Grenoble (1995).

[45] D. W. Gregg and S. J. Thomas, Momentum transfer produced by focused laser giant pulses, J. Appl. Phys. 37, 2787 (1966).

[46] E. Riet, C. J. C. M. Nillesen, and J. Dieleman, Reduction of droplet emission and target roughening in laser ablation and deposition of metals, Journal of Applied Physics 74, 2008 (1993).

[47] M. Weller, R. Herzog, M. Kilo, G. Borchardt, S. Weber, and S. Scherrer, Oxygen mobility in yttria-doped zirconia studied by internal friction, electrical conductivity and tracer diffusion experiments, Solid State Ionics 175, 1 (2004).

[48] R. W. G. Wyckoff, Crystal Structures Vol. 2 (Interscience Publishers) (1964).

[49] F. H. Spedding, A. H. Daane, and K. W. Herrmann, The crystal structures and lattice parameters of high-purity scandium, yttrium and the rare earth metals, Acta. Cryst. 9, 559 (1956).

[50] C. Chabaud-Villard, Ph.D. thesis, Universite Joseph Fourier-Grenoble (1992).

[51] Sloan handbook of thin film materials.

[52] P. Anderson, R. Reeves, and S. M. Durbin, Rf plasma sources for iii-nitrides growth: influence of operating conditions and device geometry on active species production and InN film properties, Phys. Stat. Sol. (a) 203, 106 (2006).

[53] D. X. Li, Y. Haga, H. Shida, T. Suzuki, Y. S. Kwon, and G. Kido, Magnetic properties of stoichiometric Gd monopnictides, J. Phys.: Condens. Matter 9, 10777 (1997).

[54] B. M. Ludbrook, I. L. Farrell, M. Kuebel, B. J. Ruck, A. R. H. Preston, H. J. Trodahl, S. M. Durbin, R. J. Reeves, L. Ranno, and C. Meyer, Growth and properties of epitaxial $G d N$, Journal of Applied Physics (In preparation). 
[55] H. Kronmuller, Theory of nucleation fields in inhomogeneous ferromagnets, Phys. Stat. Sol. B 144, 385 (1987).

[56] K. Khazen, H. J. von Bardeleben, J. L. Cantin, A. Bittar, S. Granville, H. J. Trodahl, and B. J. Ruck, Ferromagnetic resonance study of GdN thin films with bulk and extended lattice constants, Phys. Rev. B 74, 245330 (2006).

[57] Z. Celinski and B. Heinrich, Ferromagnetic resonance linewidth of Fe ultrathin films grown on a bcc Cu substrate, J. Appl. Phys. 70, 5935 (1991).

[58] F. Schreiber, J. Pflaum, Z. Frait, T. Muhge, and J. Pelzl, Gilbert damping and g-factor in $\mathrm{Fe}_{x} \mathrm{Co}_{1 ? x}$ alloy films, Solid State Commun. 93, 12 (1995).

[59] C. Chappert, K. L. Dang, P. Beauvillain, H. Hurdequint, and D. Renard, Ferromagnetic resonance studies of very thin cobalt films on a gold substrate, Phys. Rev. B 34, 5 (1986).

[60] R. J. Gambino, T. R. McGuire, H. A. Alperin, and S. J. Pickart, Magnetic properties and structure of $G d N$ and $G d N_{1-x} O_{x}$, Journal of Applied Physics 41, 933 (1970).

[61] H. Adachi, K. Kimura, and H. Ino, Magnetic properties of metastable h.c.p. samarium, Mat. Sci. Eng., A 182, 864 (1994).

[62] W. C. Koehler and R. M. Moon, Magnetic structures of samarium, Phys. Rev. Lett. 29, 1468 (1972).

[63] V. I. Tkach, V. F. Bashev, A. Lysenko, and I. S. Miroshnichenko, Phys. Met. Metall. 48, 183 (1980).

[64] A. M. Stewart, Paramagnetic susceptibilities of metallic samarium compounds, Phys. Rev. B 6, 5 (1972).

[65] H. Adachi, H. Ino, and H. Miwa, Effect of conduction electron polarization on the magnetism of hcp samarium metal, Phys. Rev. B 56, 1 (1997).

[66] N. H. Hong, J. Sakai, N. Poirot, and A. Ruyter, Laser ablated Ni-doped HfO2 thin films: Room temperature ferromagnets, Appl. Phys. Lett. 86, 242505 (2005). 
[67] M. Horne, P. Strange, W. M. Temmerman, Z. Szotek, A. Svane, and H. Winter, The electronic structure of europium chalcogenides and pnictides, J. Phys.: Condens. Matter 16, 28 (2004).

[68] P. G. Steeneken, New light on EuO thin films: Preparation, transport, magnetism and spectroscopy of a ferromagnetic semiconductor, Ph.D. thesis, Rijksuniversiteit Groningen (2002). 